\title{
A Chandra Virgo cluster survey of spiral galaxies. I. Introduction to the survey and a new ULX sample
}

\author{
Roberto Soria $^{1,2} \star$, Mari Kolehmainen ${ }^{3}$, Alister W. Graham ${ }^{4}$, Douglas A. Swartz ${ }^{5}$, \\ Mihoko Yukita $^{6,7}$, Christian Motch $^{3}$, Thomas H. Jarrett ${ }^{8}$, James C. A. Miller-Jones ${ }^{9}$, \\ Richard M. Plotkin ${ }^{10}$, Thomas J. Maccarone ${ }^{11}$, Laura Ferrarese ${ }^{12}$, Alexander Guest ${ }^{13}$, \\ Ariane Lançon ${ }^{3}$ \\ ${ }^{1}$ College of Astronomy and Space Sciences, University of the Chinese Academy of Sciences, Beijing 100049, China \\ ${ }^{2}$ Sydney Institute for Astronomy, School of Physics A28, The University of Sydney, Sydney, NSW 2006, Australia \\ ${ }^{3}$ Université de Strasbourg, CNRS, Observatoire Astronomique, CNRS, UMR 7550,F-67000, Strasbourg, France \\ ${ }^{4}$ Centre for Astrophysics and Supercomputing, Swinburne University of Technology, Hawthorn, VIC 3122, Australia \\ ${ }^{5}$ Astrophysics Office, NASA Marshall Space Flight Center, ST12, Huntsville, AL 35812, USA \\ ${ }^{6}$ Code 662, NASA Goddard Space Flight Center, Greenbelt, MD 20771, USA \\ ${ }^{7}$ Henry A. Rowland Department of Physics and Astronomy, Johns Hopkins University, Baltimore, MD 21218, USA \\ ${ }^{8}$ Department of Astronomy, University of Cape Town, Private Bag X3, Rondebosch, 7701, South Africa \\ ${ }^{9}$ International Centre for Radio Astronomy Research, Curtin University, GPO Box U1987, Perth, WA 6845, Australia \\ ${ }^{10}$ Department of Physics, University of Nevada, Reno, NV 89557, USA \\ ${ }^{11}$ Department of Physics, Box 41051, Science Building, Texas Tech University, Lubbock, TX 79409-1051, USA \\ ${ }^{12}$ Herzberg Astronomy and Astrophysics Research Centre, National Research Council of Canada, Victoria, BC V9E 2E7, Canada \\ ${ }^{13}$ Department of Physics and Astronomy, University College London, Gower Street, London, WC1E 6BT, UK
}

Accepted 2022 January 17. Received 2022 January 16; in original form 2021 July 28

\begin{abstract}
We present an analysis of the ultraluminous X-ray source (ULX) population in 75 Virgo cluster late-type galaxies, including all those with a star formation rate $\gtrsim 1 M_{\odot} \mathrm{yr}^{-1}$ and a representative sample of the less star-forming ones. This study is based on 110 observations obtained over 20 years with the Chandra X-ray Observatory Advanced Camera for Imaging Spectroscopy. As part of a Large Chandra Program, new observations were obtained for 52 of these 75 galaxies. The data are complete to a sensitivity of $\approx 10^{39} \mathrm{erg} \mathrm{s}^{-1}$, with a typical detection limit of $\approx 3$ $\times 10^{38} \mathrm{erg} \mathrm{s}^{-1}$ for the majority of the sources. The catalogue contains about 80 ULXs $(0.3-$ $10 \mathrm{keV}$ luminosity $>10^{39} \mathrm{erg} \mathrm{s}^{-1}$ ), and provides their location, observed flux, de-absorbed luminosity, and (for the 25 most luminous ones) simple X-ray spectral properties. We discuss the ULX luminosity function in relation to the mass and star formation rate of the sample galaxies. We show that recent models of low-mass plus high-mass X-ray binary populations (scaling with stellar mass and star formation rate, respectively) are mostly consistent with our observational results. We tentatively identify the most luminous X-ray source in the sample (a source in IC 3322A with $L_{X} \approx 6 \times 10^{40} \mathrm{erg} \mathrm{s}^{-1}$ ) as a recent supernova or its young remnant. The properties of the sample galaxies (morphologies, stellar masses, star formation rates, total X-ray luminosities from their point-source population) are also summarised.
\end{abstract}

Key words: galaxies: clusters: individual: Virgo - galaxies: spirals - X-rays: binaries - X-rays: galaxies

\section{INTRODUCTION}

The population properties of compact X-ray sources are a tracer of star formation rate (SFR) and stellar mass $\left(M_{*}\right)$ (Grimm et al. 2003; Gilfanov 2004; Mineo et al. 2012a; Fragos et al. 2013a;

\footnotetext{
^ Email: rsoria@nao.cas.cn (RS)
}

Lehmer et al. 2019). On the one hand, accreting compact objects probe the last stages of stellar evolution and collapse. On the other hand, binary systems with compact objects include the progenitors of gravitational wave mergers. Thus, studying the number and distribution of X-ray binaries provides empirical constraints to population synthesis models and theoretical predictions of binary merger rates (Belczynski et al. 2016; Kruckow et al. 2018; Spera et al. 2019). 
Reliable scaling relations between galaxy properties and the total luminosity of the X-ray binary population are also essential to estimate the relative contribution of off-nuclear compact objects (compared with massive stars and quasars) to cosmic reionization and to the heating of the intergalactic and interstellar medium at high redshift (Mirabel et al. 2011; Fragos et al. 2013b; Kaaret 2014; Artale et al. 2015; Lehmer et al. 2016).

More specifically, here we are interested in the high-luminosity end of the X-ray binary population, near and above the Eddington limit of typical stellar-mass accretors $\left(L_{\mathrm{X}}>10^{39} \mathrm{erg} \mathrm{s}^{-1}\right)$. This class of ultraluminous X-ray sources (ULXs: see Kaaret et al. 2017 for a review) is populated both by confirmed neutron stars and candidate stellar-mass black holes (BHs), but the relative contribution of the two types of accreting compact objects remains uncertain and widely debated (Bachetti et al. 2014; Walton et al. 2018; Wiktorowicz et al. 2019; Mushtukov et al. 2019). ULXs are found both in young and in old stellar environments, although the younger systems are more common, especially at highest luminosities $\left(\gtrsim 5 \times 10^{39} \mathrm{erg} \mathrm{s}^{-1}\right)$ (Swartz et al. 2004; Maccarone et al. 2007; Zhang et al. 2012; Lehmer et al. 2019). In young-population ULXs, the donor stars are usually consistent with massive blue or red supergiants (Lau et al. 2019; Lopez et al. 2020), but other types of donors with strong winds are also possible: for example, a Wolf-Rayet star, as seen in a ULX inside the Circinus galaxy (Qiu et al. 2019), or a main-sequence B-type star, as in M82 X-2 (Fragos et al. 2015). Mass transfer from massive donors may occur via classical wind, focused wind ("wind Roche-lobe-overflow"), or Roche lobe overflow (if the mass ratio between donor and accretor is below the threshold for common-envelope formation); the relative contribution of the three processes is also still actively debated (Pavlovskii et al. 2017; Artale et al. 2019; El Mellah et al. 2019; Quast et al. 2019; Wiktorowicz et al. 2021). Instead, in old-population ULXs, the donor star may be a Roche-lobe-filling low-mass or intermediatemass star; such systems would be analogous to transient low-mass $\mathrm{X}$-ray binaries. If the Roche lobe of the compact object, and therefore its accretion disk, is very large, the thermal-viscous disk instability is predicted to cause long outburst phases and super-Eddington peak luminosities (Lasota et al. 2015; Hameury \& Lasota 2020). Alternatively, even intermediate- and low-mass stars can go through evolutionary phases when they can transfer $>10^{-6} M_{\odot} \mathrm{yr}^{-1}$ onto their compact companion for a sustained period of time of $\sim 10^{5}-10^{6}$ yr (Wiktorowicz et al. 2017; Misra et al. 2020), for example when they cross the Hertzsprung gap or as they ascend the red giant or asymptotic giant branch. Moreover, in at least one case, for an oldpopulation ULX in the Virgo giant elliptical M49, the donor star is a white dwarf (Maccarone et al. 2007, 2010; Steele et al. 2014).

Most of the empirical constraints to theoretical models of ULX formation come from X-ray surveys of accreting compact objects in nearby galaxies, followed by optical/IR studies of their stellar counterparts and environments. The Chandra X-ray Observatory is the best instrument available for such surveys, thanks to its still unsurpassed sub-arcsec spatial resolution and very low background noise. Already a few years after its launch, Chandra-based surveys showed the difference between the ULX luminosity function (LF) in spiral and elliptical galaxies. The early-type-galaxy LF is an indicator of $M_{*}$ (Kim \& Fabbiano 2004; Gilfanov 2004; Zhang et al. 2012), while the late-type-galaxy LF is mainly proportional to SFR (Grimm et al. 2003; Mineo et al. 2012a); the young-population LF is a power-law with an exponential cut-off at $L_{\mathrm{X}} \approx 2 \times 10^{40} \mathrm{erg} \mathrm{s}^{-1}$ (Swartz et al. 2011; Mineo et al. 2012a). More generally, the LF of any given galaxy is a mix of the two components (Lehmer et al. $2019)$ and depends on the specific SFR ( $\left.\mathrm{SSFR} \equiv \mathrm{SFR} / M_{*}\right)$. There is an additional smaller dependence of the high mass X-ray binary (HMXB) population on the metal abundance of the host galaxy, in the sense that metal-poor galaxies contain more luminous HMXBs (Prestwich et al. 2013; Brorby et al. 2014; Kovlakas et al. 2020; Lehmer et al. 2021).

There are now a few hundred nearby galaxies observed by Chandra deep enough to reach a completeness limit of about $10^{39}$ $\mathrm{erg} \mathrm{s}^{-1}$ or below, which can be used for ULX population studies (for example, a sample of about 300 galaxies within $40 \mathrm{Mpc}$ was used by Kovlakas et al. 2020 for their study of the ULX population). Surveys of nuclear X-ray sources suspected of being intermediatemass BHs have also been conducted with archival Chandra data (e.g., Chilingarian et al. 2018). A caveat is that archival Chandra targets are biased in favour of "interesting" galaxies, sometimes with higher-than-average X-ray luminosities or already known to contain particularly luminous ULXs discovered by earlier X-ray telescopes. This may affect the inferred scalings of the LF with sSFR. For this work, we chose instead a physically selected sample of galaxies, representative of the spiral population in the Virgo Cluster. The methodology of our sample selection is described in Section 2.1.

The Virgo Cluster is an interesting environment for a pointsource X-ray population study in galaxies with a range of Hubble types and star-forming activity. It is the richest and biggest galaxy cluster in the local universe, with a total mass (gas, stars and dark matter) $M_{\mathrm{tot}} \approx 6-8 \times 10^{14} M_{\odot}$ (Kashibadze et al. 2020, 2018; Shaya et al. 2017; Planck Collaboration 2016). It is close enough ( $d=16.1 \pm 1.0 \mathrm{Mpc}$ for its central galaxy, M 87, in the weightedaverage estimate of de Grijs \& Bono 2020) that Chandra imaging observations can reach $L_{\mathrm{X}} \approx$ a few $10^{38} \mathrm{erg} \mathrm{s}^{-1}$ in $\lesssim 10 \mathrm{ks}$ and are not affected by source confusion. Virgo is also just close enough to permit at least a detection and possibly a rough colour identification of the optical counterparts; at a distance modulus of $31 \mathrm{mag}$, the typical optical brightness of young-population ULXs $\left(M_{V} \sim-7\right.$ mag to -4 mag: Ambrosi \& Zampieri 2018; Gladstone et al. 2013; Tao et al. 2011) corresponds to $V \sim 24-27 \mathrm{mag}$. Moreover, Virgo already has a huge wealth of multiband datasets, which enable us to determine the SFR and $M_{*}$ of many of its galaxies.

A sample of 100 early-type Virgo galaxies (including E, dE and S0 morphological types) was observed by Chandra as part of the "Active galactic nucleus MUltiwavelength Survey of Early-Type Galaxies" (AMUSE) Virgo study (Gallo et al. 2008, 2010). The sample was selected to coincide with the Hubble Space Telescope (HST) Virgo Cluster Survey (Côté et al. 2004). In turns, the HST sample was selected from early-type galaxies with blue brightness $B_{T}<16 \mathrm{mag}$, a published central velocity dispersion, and no peculiar properties such as mergers or close interactions. The total stellar mass $\left(M_{*}\right)$ of the AMUSE-Virgo sample was $M_{*} \approx 6 \times 10^{12} M_{\odot}$ (derived by Gallo et al. 2008, 2010, from the $g_{0}$ and $z_{0}$ luminosities, according to the relation of Bell et al. 2003). The Chandra coverage was designed to be complete down to $\approx 4 \times 10^{38} \mathrm{erg} \mathrm{s}^{-1}$. The AMUSE X-ray survey provided an exceptional view of nuclear BH activity and Eddington ratio in "normal" early-type galaxies (Gallo et al. 2010; Miller et al. 2012; Graham \& Soria 2019), as well as a sample of bright off-nuclear sources.

From the AMUSE data, Plotkin et al. (2014) found 55 sources with an apparent $0.3-10 \mathrm{keV}$ luminosity $L_{\mathrm{X}}>10^{39} \mathrm{erg} \mathrm{s}^{-1}$, in the Virgo ellipticals. However, only 13 of them had an apparent (projected) luminosity higher than $\approx 2 \times 10^{39} \mathrm{erg} \mathrm{s}^{-1}$, and those brighter sources were statistically consistent with background AGN 


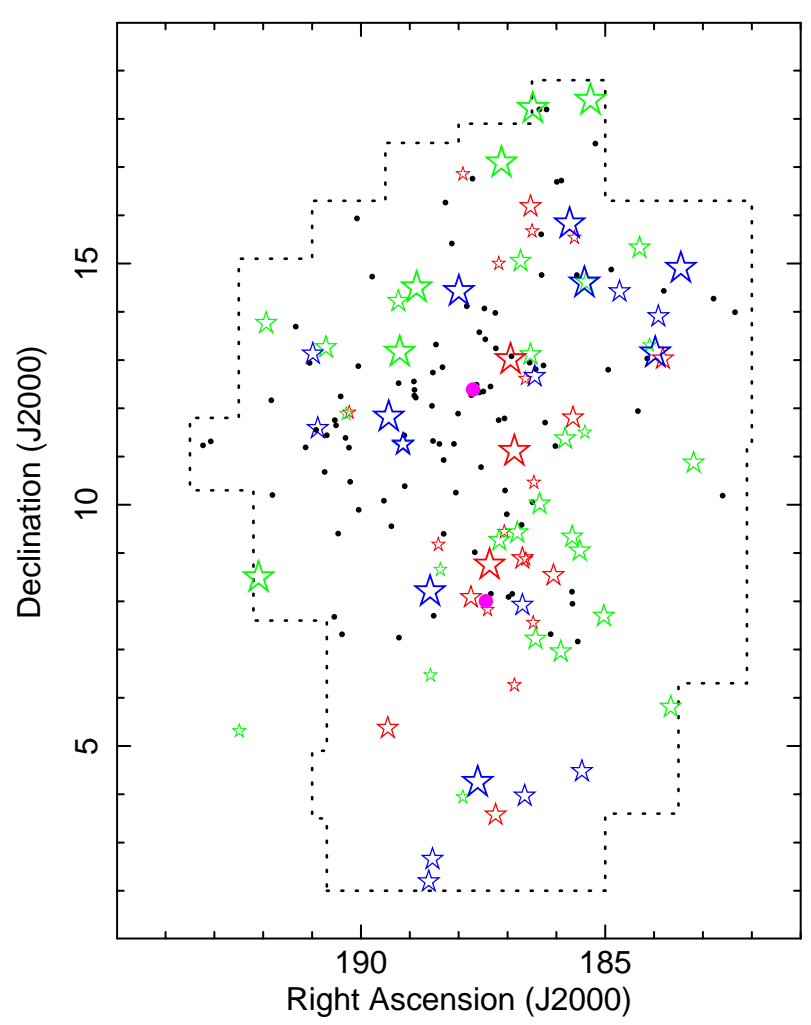

Figure 1. Sky map of the Virgo galaxies. Stars mark the positions of the 75 spirals in our study. Black dots mark the position of the 100 AMUSE-Virgo early-type galaxies; the two magenta dots represent M 87 (further to the north) and M 49 (further to the south). Small-sized stars represent spirals in our sample with low stellar masses $\left(\log \left(M_{*} / M_{\odot}\right)<9.8\right)$; intermediatesized stars represent intermediate masses $\left(9.8 \leq \log \left(M_{*} / M_{\odot}\right)<10.4\right)$; and large-sized stars represent the most massive spirals $\left(\log \left(M_{*} / M_{\odot}\right) \geq\right.$ 10.4). Red-coloured stars denote galaxies with low SFR (SFR $<0.5 M_{\odot}$ $\mathrm{yr}^{-1}$, according to the WISE $12-\mu \mathrm{m}$ proxy); green-coloured stars are for intermediate rates $\left(0.5 M_{\odot} \mathrm{yr}^{-1} \leq \mathrm{SFR}<1.5 M_{\odot} \mathrm{yr}^{-1}\right)$; blue-coloured stars are for high rates (SFR $\geq 1.5 M_{\odot} \mathrm{yr}^{-1}$ ). (The choice of the three bands in stellar mass and SFR is arbitrary, for illustration purposes.) A dotted line marks the boundaries of the Virgo Cluster Survey (Binggeli et al. 1984). The only spiral in our sample that is outside the VCC region is included in the Extended VCC (Kim et al. 2014) and is a probable member based on its redshift-independent distance and recession speed.

projected inside the $D_{25}$ area $^{1}$ of the Virgo galaxies. Plotkin et al. (2014) concluded that in the old stellar population, there is $\approx 1$ ULX per $M_{*} \approx 1.6 \times 10^{11} M_{\odot}$. This is consistent with the expected LF of low mass X-ray binaries (LMXBs) in early-type galaxies (Zhang et al. 2012).

To have a more complete understanding of the nuclear and off-nuclear X-ray sources in Virgo, we must integrate the existing study of its elliptical galaxies with a new study of its spiral galaxies. Virgo is a spiral-rich cluster, a signature of an early evolutionary stage (clusters such as Coma, by contrast, are more evolved and spiral-poor). However, many of the Virgo Cluster's spirals have lost $\mathrm{HI}$ gas via ram pressure stripping and tidal stripping, and as a result, the sSFR of Virgo spirals tends to be lower than for field spirals of similar Jeans-Lundmark-Hubble type and mass (see Boselli \& Gavazzi 2006 for a detailed review and discussion

1 The $D_{25}$ region of a galaxy is defined as the 25th magnitude B-band isophote.

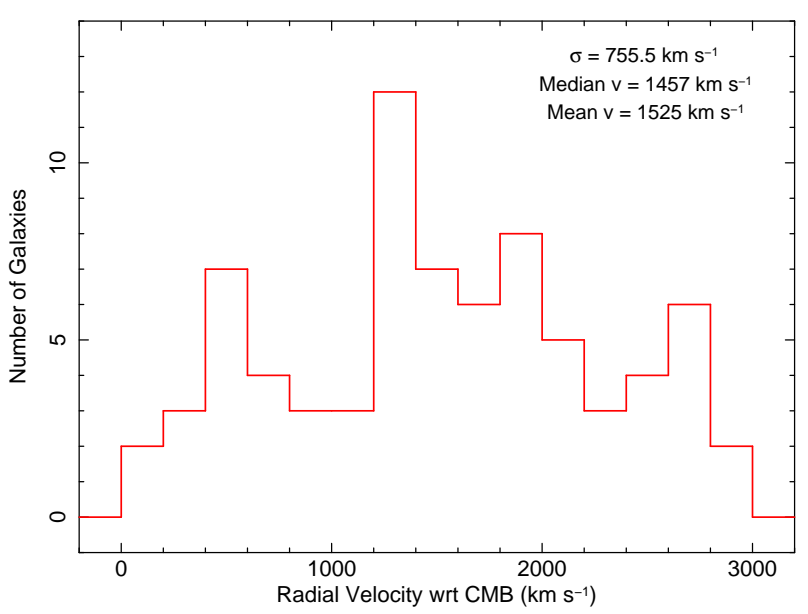

Figure 2. Distribution of radial velocities with respect to the cosmic microwave background, for the galaxies in our sample. The mean and median velocities, and the Gaussian standard deviation, are also labelled in the plot.

of such issues, and Graham 2019 for a comprehensive discussion of morphological types). For the off-nuclear X-ray sources, Virgo spirals contain two populations of stellar-mass objects. First, a sample of LMXBs in spirals; the number and luminosity of such sources as a function of stellar mass may not be the same as for the LMXBs in ellipticals. Second, a sample of young high mass $\mathrm{X}$-ray binaries (HMXBs), in the galaxies with a higher SFR. Third, for centrally-located, i.e., nuclear, BHs, a Chandra study can reveal, for example, the difference in the Eddington ratio distribution between galaxies dominated by hot gas (early-type) or cold gas (late-type). It can also reveal the presence of candidate X-ray-bright intermediate-mass $\mathrm{BHs}$ in the nuclei of spiral galaxies with a small bulge or no bulge (as predicted by galaxy/BH scaling relations), which can then be the subject of subsequent multiband investigations (Chilingarian et al. 2018; Mezcua et al. 2018). Furthermore, the unsurpassed sub-arcsec astrometric accuracy and sharp pointspread function of Chandra enables a search for slightly off-centre, "wandering" nuclear BHs (Khan et al. 2020; Ricarte et al. 2021). In this paper, we will mostly focus on off-nuclear sources, and we will leave the analysis of the nuclear BHs and candidate intermediatemass BHs to separate work.

By contrast with the early-type galaxies, the archival Chandra coverage of late-type Virgo galaxies was, until recently, surprisingly scant. Less than 30 Virgo spirals had been observed before 2017, an insufficient number for population studies. Thus, we planned a larger survey and successfully applied for $550 \mathrm{ks}$ of Chandra time (Cycle 18 Large Program, PI R. Soria). In the next Section, we describe the population properties of the Chandra sample. In Section 3, we outline our X-ray data analysis. In Section 4, we present the results for the off-nuclear source population, and place it in the context of the properties of their host galaxies. A census of the nuclear sources, a study of the diffuse X-ray emission in the most star-forming galaxies, and an investigation of the multiband counterparts will be presented in further work.

\section{A CHANDRA SAMPLE OF VIRGO SPIRALS}

\subsection{Selection criteria}

The selection of targets for our study resulted from a necessary compromise between various ideal requirements: a large number 
of galaxies, particularly with high SFR (because that is the other extreme of the range of galaxy activity compared to ellipticals; that is where we expect to find the most numerous and luminous X-ray binaries), but also a good sampling of all morphological types from early-type (large bulge) to late-type (small or no bulge) spirals and with a sufficiently large range of specific SFR (so as to differentiate between galaxies dominated by LMXBs, and those dominated by HMXBs). Each target should be observed deep enough to be complete at least down to $10^{39} \mathrm{erg} \mathrm{s}^{-1}$ (in most cases, we required a detection limit of $\approx 3 \times 10^{38} \mathrm{erg} \mathrm{s}^{-1}$ ). Finally, the total exposure time has to be kept within plausible limits.

There are about 70 Virgo spirals with SFR $\gtrsim 0.3 M_{\odot} \mathrm{yr}^{-1}$, but the precise list depends on the various alternative proxies for the SFR (H $\alpha$ luminosity, or radio continuum, or far-IR, or far-UV plus 24- $\mu \mathrm{m}$ luminosity) as well as the adopted distance to the galaxies within Virgo. Moreover, some of the SFR studies we use for the analysis in this paper were not available at the time of our sample selection. With these caveats, we selected a representative sample ${ }^{2}$ of about 60 galaxies with $\mathrm{SFR} \gtrsim 0.3 M_{\odot} \mathrm{yr}^{-1}$, plus about 15 galaxies with a lower SFR, but which were either already in the Chandra archives or were in the same field of view of another target with a higher SFR. In total, we compiled a list of 75 spirals: a sample large enough to be representative of the population of actively starforming galaxies in Virgo, and to provide a sizeable population of ULXs. In particular, we included all the $\approx 20$ spirals with SFR $\gtrsim 1$ $M_{\odot} \mathrm{yr}^{-1}$. Of the 75 galaxies in the sample, 46 were observed for the first time by Chandra through our Large Program. For another six galaxies, new observations were combined with a previous snapshot observation in order to reach the required detection limit, though any detected sources were subsequently analyzed separately for each observation. Finally, the remaining 23 galaxies already had sufficiently deep archival observations. In the rest of this Section we summarize the main properties of our sample. We will then describe our data analysis and main results, in particular for the ULX census. A more detailed analysis of the most interesting or peculiar individual sources will be presented in follow-up papers.

\subsection{Cluster membership}

Seventy-four out of our 75 galaxies (Figure 1) are included in the Virgo Cluster Catalog (VCC) of Binggeli et al. (1984), which is often used as the standard reference for a galaxy census in this cluster. The only galaxy in our sample that falls outside the VCC footprint is NGC 4713. However, it is included in the Extended Virgo Cluster Catalog (Kim et al. 2014), and it is a likely cluster member based on its recession speed and redshift-independent distance.

A VCC classification does not of course guarantee that a galaxy is physically associated with Virgo. This is especially true for spirals, which are more spread out around the edges of the cluster, while ellipticals are more concentrated in the central region, around M 87 and M 49. As a test that all 75 galaxies are at least plausible cluster members, we inspected their radial velocity distribution with respect to the cosmic background, using the values of $v_{3 \mathrm{~K}}$ reported in

\footnotetext{
2 We do not claim that the sample is complete within a certain threshold; simply that it is well representative of most spirals with high SFR. All the $\approx 20$ spirals with SFR $\gtrsim 1 M_{\odot} \mathrm{yr}^{-1}$ are included. Among those with a SFR between $\approx 0.4-1 M_{\odot} \mathrm{yr}^{-1}$, we are aware of the following six Virgo galaxies missing from the sample: NGC 4294, NGC 4634, NGC 4409, IC 3476, NGC 4630, and NGC 4207. A case can be made also for NGC 4383 (with an uncertain classification between S0 and Sa peculiar), and NGC 4651 (located at the northern edge of Virgo, probably a member)
}

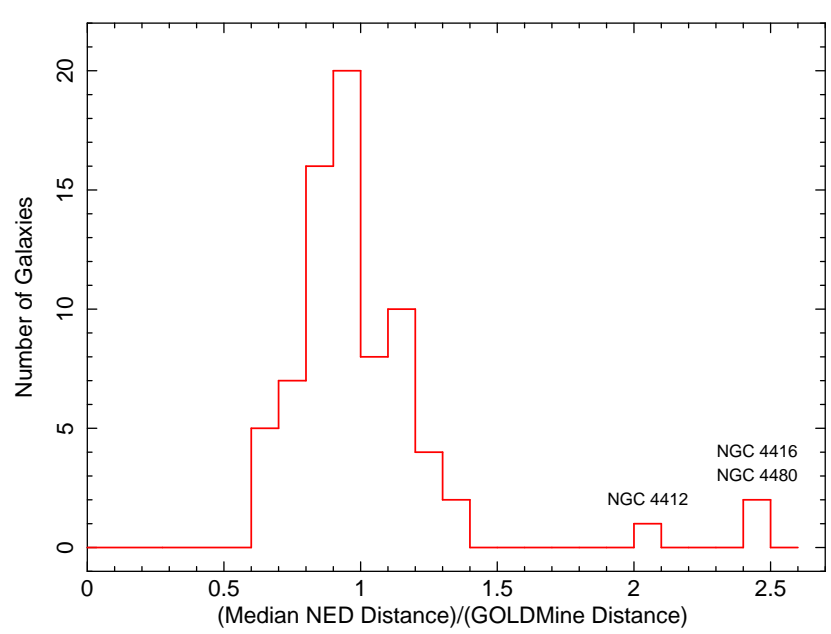

Figure 3. Ratio between the median redshift-independent distance (from NED) and the conventional Virgo sub-structure distance (17, 23 or $32 \mathrm{Mpc}$ ), for the 75 galaxies in our sample. Three galaxies with very discrepant distance measurements are labelled in the plot and discussed in Section 2.3.

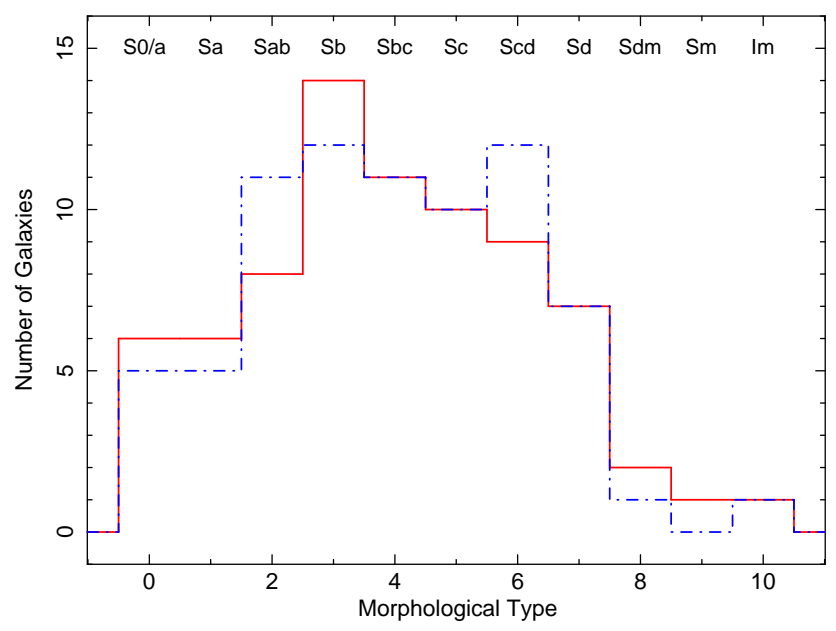

Figure 4. Morphological type distribution of the galaxies in our sample, expressed with the "Hubble stage" index $T$ (horizontal axis) (de Vaucouleurs 1959; de Vaucouleurs et al. 1991) and with the corresponding HubbleSandage-de Vaucouleurs classification (label over each histogram bin). The solid red histogram is for the galaxy morphologies reported in NED; the dash-dotted blue histogram is for the morphological classification of HyperLEDA.

HyperLEDA $^{3}$. We obtain a broad, structured, tri-modal distribution (Figure 2), which spans $\approx 3000 \mathrm{~km} \mathrm{~s}^{-1}$, consistent with the findings of Binggeli et al. (1987) from the whole VCC. A few additional galaxies with Chandra archival data are physically located inside the VCC footprint (thus, they do have a VCC number) but their radial velocity is $>3000 \mathrm{~km} \mathrm{~s}^{-1}$, which locates them most likely behind the cluster: we did not include those outliers in our sample of 75 candidate members.

\subsection{Distances}

Recession speed is not a useful proxy for the distance of individual Virgo galaxies, because it is comparable to the proper motion inside

3 http://leda.univ-lyon1.fr 
Table 1. Main properties of the sample of 75 Virgo spirals

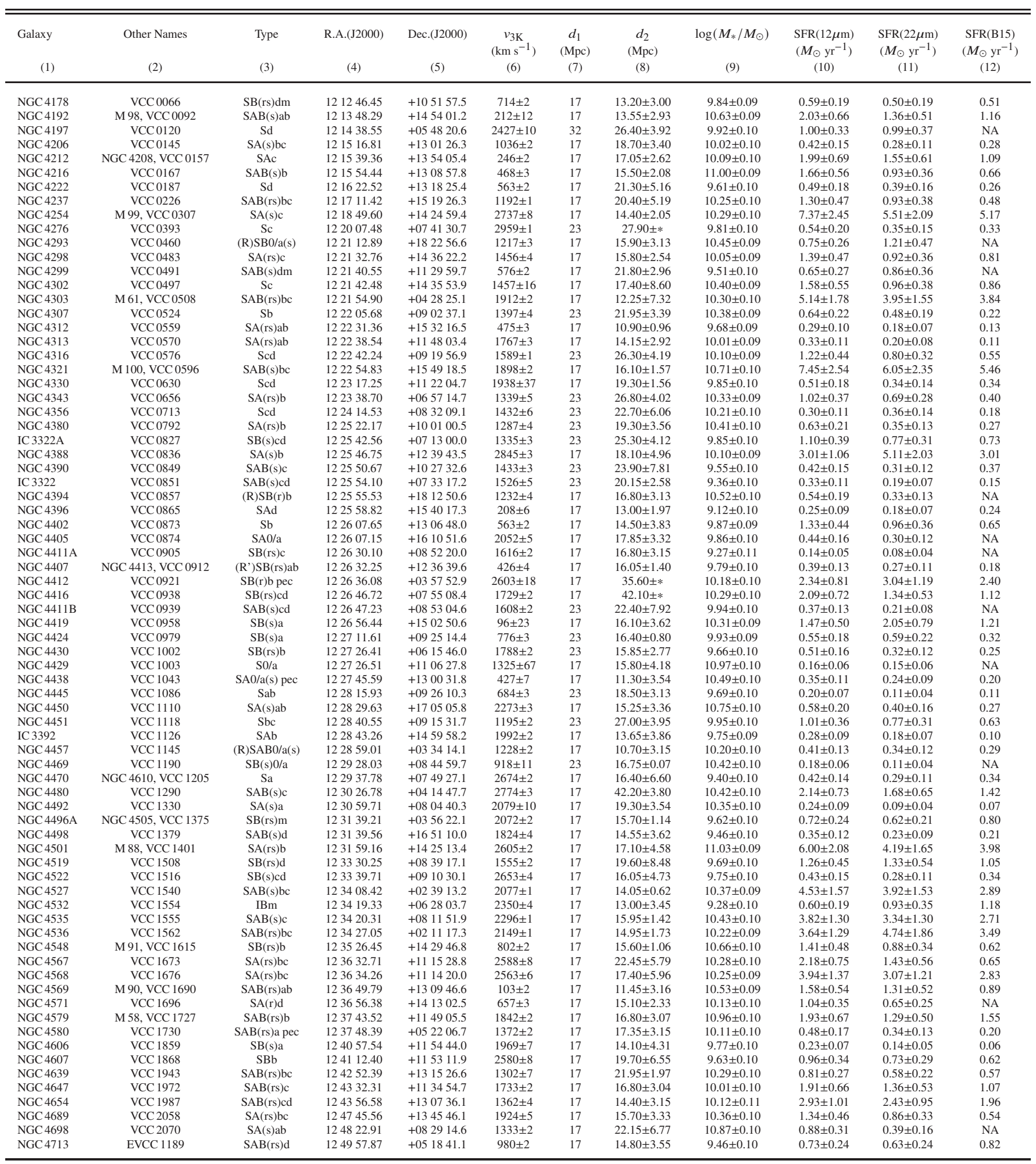

Col. (3): from NED. Col. (6): radial velocity with respect to the cosmic microwave background (from HyperLeda). Col. (7): galaxy distance assuming the conventional structure of the Virgo Cluster, that is: $17 \mathrm{Mpc}$ for the Virgo A subclump and the N, S and E clouds; $23 \mathrm{Mpc}$ for the Virgo B subclump; $32 \mathrm{Mpc}$ for the W and M clouds (as adopted, for example, by Gavazzi et al. 1999, Boselli et al. 2015, and the GOLDMine database). Col. (8): median

redshift-independent distance from NED, and standard deviation of the published values. When a galaxy has Cepheid distance measurements, we used the median and standard deviation of those values only. Col. (9): stellar mass, derived from WISE W1 and W2 fluxes, as calibrated by Cluver et al. (2014) (see also Jarrett et al. 2019). The median NED distances were adopted. Col. (10): SFR from the WISE W3 flux, derived with the calibration from Cluver et al. (2017) (see Eq. 2), and assuming median NED distances. Col. (11): SFR from the WISE W4 flux, derived with the calibration from Cluver et al. (2017) (see Eq. 3). Col (12): average SFR computed by Boselli et al. (2015), from four different proxies. We have rescaled their published values (originally based on sub-structure distances) to our median NED distances. The $1-\sigma$ errors for the SFRs in Col (12) are $\approx 20 \%$ of the values. 


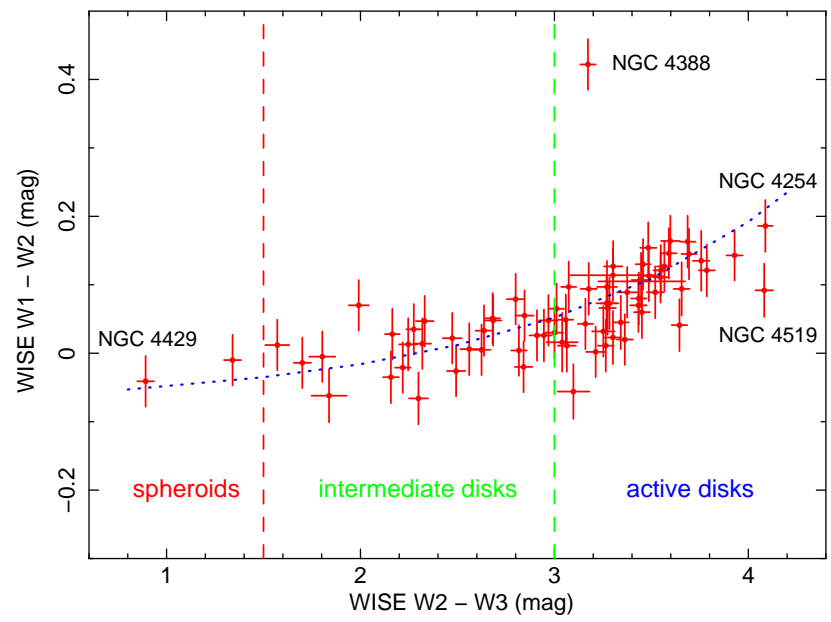

Figure 5. WISE classification of the galaxies in our sample, following Jarrett et al. (2019). All the galaxies follow the "star formation sequence" (dotted blue line) of normal galaxies, except for NGC 4388 (a Seyfert 2 galaxy), in which the $W 1$ colour is contaminated by the active nucleus. Spiral galaxies on the left of the sequence are more bulge-dominated, while those on the right have a younger and more active disk. The most bulge-dominated galaxy in our sample is NGC 4429 (classified as S0/a in HyperLEDA), while the most disky are NGC 4519 and NGC $4254=$ M 99 .

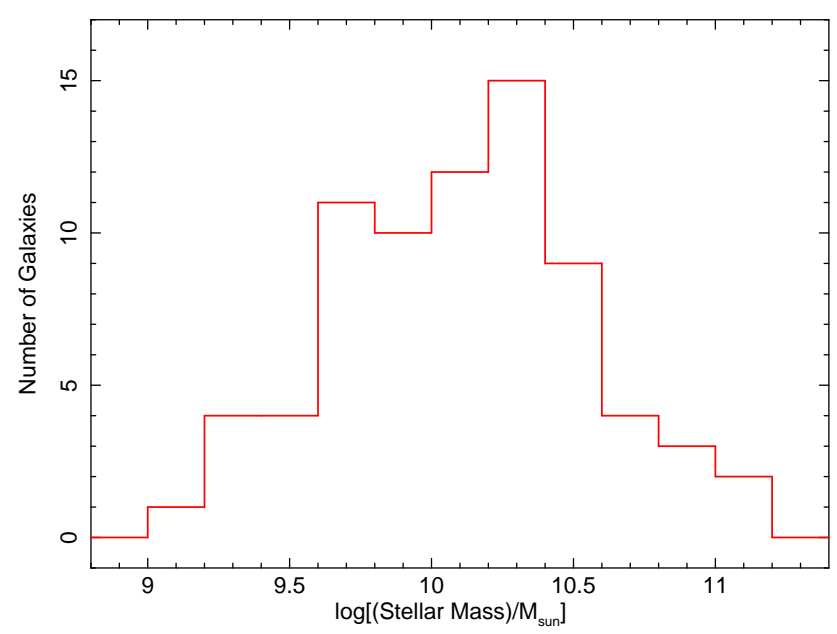

Figure 6. Stellar mass distribution, from the mid-infrared luminosity (WISE colours), assuming the median redshift-independent distances from NED (Section 2.3).

the cluster. There are two common alternative ways of allocating distances in Virgo cluster surveys. One method is to divide the cluster into sub-clusters and "clouds", determine an average distance for each substructure, and attribute that distance to all the galaxies in the substructure. The most commonly used subdivision (Gavazzi et al. 1999, 2002; Boselli et al. 2015) consists of a cluster A around M 87, located at an assumed (partly conventional) distance of $17 \mathrm{Mpc}$; a cluster B around the position of M 49, at 23 Mpc (but M 49 itself is only projected in front of cluster $\mathrm{B}$, and is allocated a distance of $17 \mathrm{Mpc}$ ); clouds N, S, E at $17 \mathrm{Mpc}$; clouds W, M at $32 \mathrm{Mpc}$ (see Fig. 8 in Gavazzi et al. 1999 for a definition of the conventional sky boundaries of those regions). This is also the convention adopted in the Galaxy On-Line Database MIlano NEtwork (GOLDMine ${ }^{4}$, Gavazzi et al. 2003).

In terms of our sample, 29 out of our 75 spirals belong to cluster A, 15 to cluster B, another 15 to cloud S, 8 to cloud N, 7 to cloud $\mathrm{E}$ and 1 to cloud W. Thus, in this classification, 59 of our galaxies have a distance of $17 \mathrm{Mpc}, 15$ are at $23 \mathrm{Mpc}$, and one (NGC 4197) is at $32 \mathrm{Mpc}$ (see Table 1, Col. 7). The relative high number of targets in clouds $\mathrm{S}$ (in particular, at its southern end, for Declination $\lesssim 5^{\circ}$ ) and $\mathrm{N}$ is not surprising, as those two infalling subgroups consist of spirals for $\approx 80 \%$ of their members (Gavazzi et al. 1999), while the ellipticals are more concentrated near the centre.

While attractive for its simplicity, the main shortcoming of this classification is that the projected sky location inside a subgroup boundary is no guarantee that a particular galaxy is not in the foreground or background: the clouds and sub-clusters themselves may be several Mpc thick. An example of this issue is discussed in Boselli et al. (2002), who note the presence of gas-rich spirals in cluster A alongside the more common (and expected) gas-deficient ones. They suggest that the gas-rich galaxies are located $\approx 10 \mathrm{Mpc}$ behind the core of Virgo, at the outskirts of the cluster, where they have not yet been ram-pressure stripped of their gas by the intracluster medium. One of the best examples of such gas-rich galaxies is NGC 4480 .

The alternative choice, adopted here, for the galaxy distances is to use the database of redshift-independent measurements in the literature, as reported in the NASA/IPAC Extragalactic Database $\left(\mathrm{NED}^{5}\right)$. Such distances come from a variety of methods, and it is usually impossible to check the various assumptions or limitations that affected the original analyses. When Cepheid distances were available (for 9 of the 75 galaxies, namely NGC 4321, NGC 4424, NGC 4496A, NGC 4527, NGC 4535, NGC 4536, NGC 4548, NGC 4571 and NGC 4639), we used the median and standard deviation of those measurements. Otherwise, we adopted the median and standard deviation of all NED values (mostly Tully-Fisher measurements) after first inspecting the histogram of redshift-independent distances, removing outliers and re-deriving the median and its uncertainty. These are recorded in Table 1, Col. 8. Finally, we compared the median NED distances with the substructure distance (Figure 3 ). The scatter around a ratio of 1 is expected, given the typical standard deviation of several Mpc in the measured distances for each galaxy, and the intrinsic thickness of the substructures (also several Mpc). There are only three outliers (NGC 4412, NGC 4416, NGC 4480, labelled in Figure 3) which have Tully-Fisher distances ( $\sim 40 \mathrm{Mpc}$ ) much larger than their substructure distances $(17 \mathrm{Mpc})$. We do not have elements to decide at this stage which set of distances is more reliable, and whether those three galaxies are Virgo cluster members. The uncertainty in the distance to NGC 4480, in particular, affects our census of the most luminous ULXs, because the most luminous point-like source in that galaxy has either an X-ray luminosity $\sim 10^{40} \mathrm{erg} \mathrm{s}^{-1}$ or barely above $10^{39} \mathrm{erg} \mathrm{s}^{-1}$ depending on the choice of distance.

\subsection{Morphology and stellar masses}

Our sample includes a good balance of early- and late-type spirals (Figure 4). About half of the galaxies are of type S0/a to Sbc, and the other half from Sc to Im (with a little discrepancy between the classification in NED and HyperLEDA). There are eight Messier

\footnotetext{
4 http://goldmine.mib.infn.it

5 https://ned.ipac.caltech.edu
} 


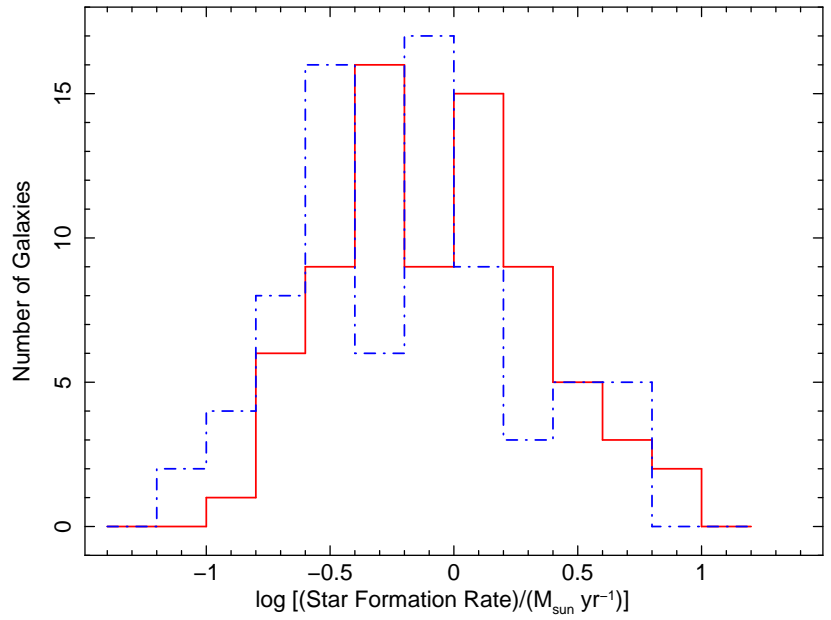

Figure 7. Solid red histogram: SFR distribution, inferred from the WISE 12- $\mu \mathrm{m}$ luminosity (Jarrett et al. 2019). Dash-dotted blue histogram: SFR distribution from the WISE 22- $\mu$ m luminosity with the scaling of Jarrett et al. (2019).

galaxies (Messier 1781) in our sample: M 58, M 61, M 88, M 90, M91, M 98, M99, M 100.

An alternative classification of spiral galaxies is based on their mid-infrared colours, as measured by the Wide-field Infrared Survey Explorer (WISE; Wright et al. 2010). All 75 galaxies in our sample have been imaged and characterized with WISE: this is part of a larger project called the WISE Extended Source Catalog 6 (WXSC). WISE photometry and global properties for the 100 largest galaxies in the sky (first data release of the WXSC) was published by Jarrett et al. (2019): that sample already included a few of our 75 Virgo targets. For all other galaxies, we used WISE results that will be published in follow-up WXSC data releases. For a detailed technical description of how the total WISE luminosity of each galaxy was measured, see Jarrett et al. (2019). In short, the steps followed by the WSXC team were the following. First, Galactic foreground stars projected onto a galaxy were identified and removed. Then, a local background was computed from an elliptical annulus, with inner and outer radii located $\approx 30 \%$ and $\approx 50 \%$ beyond the maximum extent of the galactic disk. Foreground stars were also masked from the background annulus. Each galaxy was assumed to have a constant ellipticity and to be axisymmetric. To estimate the total flux of the galaxies, Jarrett et al. (2019) simultaneously fit two Sérsic functions (bulge + disk) to the surface brightness profile. See Jarrett et al. (2019) for details.

WISE has four channels: W1, W2, W3 and W4, corresponding to the $3.4-\mu \mathrm{m}, 4.6-\mu \mathrm{m}, 12-\mu \mathrm{m}$ and $22-\mu \mathrm{m}$ bands, respectively. Plotting the $W 1-W 2$ colours against the $W 2-W 3$ colours defines a "star formation sequence" of normal galaxies (Jarrett et al. 2017, 2019). All our galaxies are located along that sequence (Figure 5), except for NGC 4388 , which is $\approx 0.3$ mag above the sequence, in a region typically occupied by Seyfert and low-luminosity AGN (Mingo et al. 2016; Huang et al. 2017), which is indeed the case for NGC 4388. The reddest galaxy along the mid-infrared star formation sequence is NGC 4429 (classified as S0/a in HyperLEDA); the bluest one is NGC $4254=$ M 99 (an Sc galaxy).

Stellar masses $\left(M_{*}\right)$ were derived from the mid-infrared luminosity. Specifically, we used the relation from Cluver et al. (2014),

6 https://vislab.idia.ac.za/research-wxsc

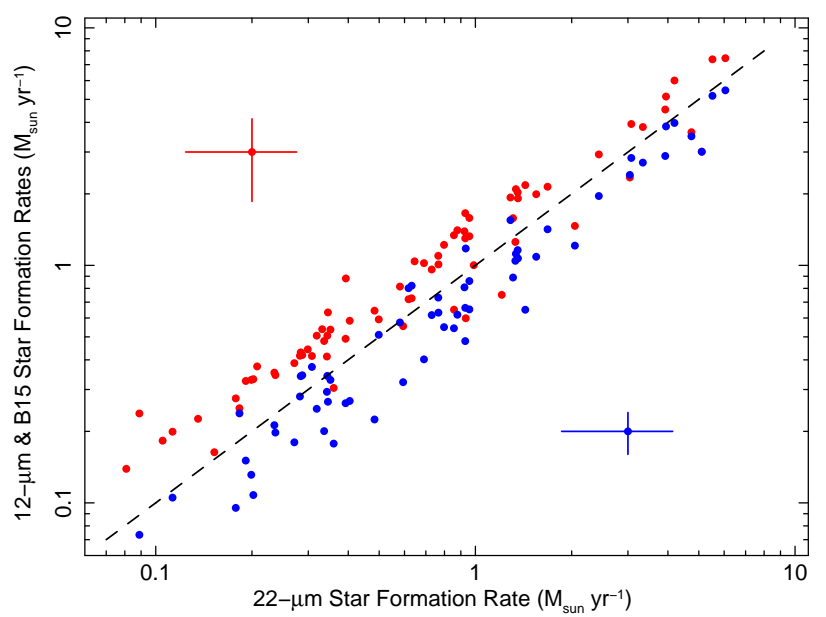

Figure 8. Comparison between the SFRs derived from the $12-\mu \mathrm{m}$ luminosities, the 22- $\mu \mathrm{m}$ luminosities, and the average SFRs from Boselli et al. (2015), B15. The dashed line is the one-to-one relation. On average, the $22-\mu \mathrm{m}$ SFRs are $\approx 20 \%$ higher than the B15 values (plotted in blue) and $\approx 20 \%$ lower than the $12-\mu$ m values (plotted in red). The difference between the three estimates is relatively more prominent for low SFRs. Typical uncertainties on the WISE SFRs from Equations 2 and 3 are around 33-38 percent (see Table 1), and around 20 percent for the B15 values. The red datapoint in the top left sector of the plot and the blue datapoint in the bottom right sector are labels, displayed to represent the typical size of the error bars.

based on the WISE photometry; see also Jarrett et al. (2013, 2019):

$\log \left(M_{*} / L_{\mathrm{W} 1}\right)=-2.54(\mathrm{~W} 1-\mathrm{W} 2)-0.17$,

where $M_{*}$ is in solar units, W1 and $\mathrm{W} 2$ are the magnitudes in the 3.4- $\mu \mathrm{m}$ and 4.6- $\mu \mathrm{m}$ bands, respectively, and $L_{W 1}$ is the luminosity in the W1 band in units of solar luminosity. (The absolute magnitude of the Sun in the W1 band is $M_{\mathrm{W} 1, \odot}=3.24 \mathrm{mag}$ ). This definition of $M_{*}$ is in excellent agreement (better than $10 \%$ ) with the mass relation used by Graham et al. (2019) in their study of Virgo spirals, namely $M_{*} / L_{K}=0.6$, where $L_{K}$ is the $K$-band luminosity. (The good agreement between the $K$-band and WISE mass values holds also for NGC 4388, the galaxy where the WISE photometry was moderately affected by an AGN, as noted earlier). The main reason we adopt the WISE scaling rather than the $K$-band scaling in this paper is because we have also used the WISE band luminosities as a proxy for the SFR (Section 2.5).

From the mass values listed in Table 1 (Column 9), we see that $\approx 3 / 4$ of our sample galaxies have masses between $\approx 5 \times 10^{9} M_{\odot}$ and $\approx 5 \times 10^{10} M_{\odot}$ (Figure 6 ). The four most massive galaxies are NGC 4216, NGC 4429, NGC 4501 (= M 88) and NGC 4579 (= M 58): all four of them have $M_{*} \approx 1 \times 10^{11} M_{\odot}$. The mass values scale with the square of the assumed distances. The values listed in Table 1 and adopted for subsequent analysis are for the median NED distances, after correcting for outliers. The error range for each galaxy includes only the photometric error in WISE W1 and W1 W2, not the systematic uncertainty in the distance; it also does not include the scatter around the best-fitting relation, which we estimate as \pm 0.15 dex, from Fig. 6 of Cluver et al. (2014). In total, the stellar mass of our galaxy sample is $M_{* \text {,tot }} \approx 1.5 \times 10^{12} M_{\odot}$, if we assume the redshift-independent NED distances, or $M_{*, \text { tot }} \approx 1.7 \times 10^{12} M_{\odot}$ if we use the substructure distances. The main reason for computing a total stellar mass is that we shall estimate the number of LMXB ULXs per unit stellar mass in our sample of star-forming galaxies (Section 4.4, Section 5), and compare it both with the value found in 
the early-type galaxies of the AMUSE sample (Plotkin et al. 2014) and with theoretical predictions of X-ray binary populations.

\subsection{Star formation rates}

To characterize the star formation properties of the sample, we looked for SFR proxies that were available for all 75 galaxies, for consistency. The luminosity in the WISE bands W3 $(12 \mu \mathrm{m})$ and $\mathrm{W} 4(22 \mu \mathrm{m})$ can be used as a proxy for the total infrared luminosity and, therefore, for the SFR (Jarrett et al. 2013; Cluver et al. 2014, 2017; Jarrett et al. 2019). The relations calibrated by Cluver et al. (2017) are:

$$
\begin{aligned}
\log \left(\frac{\mathrm{SFR}}{M_{\odot} \mathrm{yr}^{-1}}\right)= & (0.873 \pm 0.021) \log \left(\frac{v L_{12 \mu \mathrm{m}}}{L_{\odot}}\right) \\
\log \left(\frac{\mathrm{SFR}}{M_{\odot} \mathrm{yr}^{-1}}\right)= & (0.900 \pm 0.027) \log \left(\frac{v L_{22 \mu \mathrm{m}}}{L_{\odot}}\right) \\
& -(7.87 \pm 0.24)
\end{aligned}
$$

for the W3 and W4 bands, respectively. In Equations (2-3), $v$ is the central frequency of the band, and the luminosity $v L_{v}$ is normalized to the bolometric luminosity of the Sun $\left(L_{\odot}=3.83 \times 10^{33} \mathrm{erg}\right.$ $\mathrm{s}^{-1}$ ), not its band-limited luminosity. The contribution of the stellar continuum was subtracted from $L_{12 \mu \mathrm{m}}$ and $L_{22} \mu \mathrm{m}$ as described in Cluver et al. (2017). A Kroupa initial mass function (IMF) was adopted (Kroupa 2001).

The WISE SFRs are presented in Table 1 (columns 10 and 11) and are graphically shown in the upper panel of Figure 9. The total $\mathrm{SFR}_{12 \mu \mathrm{m}}$ of the sample is $(104 \pm 6) M_{\odot} \mathrm{yr}^{-1}$, if we adopt redshiftindependent distances, or $(115 \pm 8) M_{\odot} \mathrm{yr}^{-1}$ for substructure distances. The total $\mathrm{SFR}_{22 \mu \mathrm{m}}$ is $(86 \pm 6) M_{\odot} \mathrm{yr}^{-1}$ or $(95 \pm 7) M_{\odot} \mathrm{yr}^{-1}$ in the two cases. (Note that the two sets of WISE-derived SFRs scale as $d^{1.75}$ and $d^{1.80}$, respectively, as a function of assumed galaxy distances, while stellar masses scale as $d^{2}$ ). It is clear (Figure 7) that there is a systematic offset in the distribution of $\mathrm{SFR}_{12 \mu \mathrm{m}}$ compared with the corresponding values of $\mathrm{SFR}_{22 \mu \mathrm{m}}$. This is evident in particular at lower SFRs $\left(\leqslant 0.3 M_{\odot} \mathrm{yr}^{-1}\right)$, where $\mathrm{SFR}_{12 \mu \mathrm{m}}$ tends to be $\sim 1.5-2$ times higher than $\operatorname{SFR}_{22 \mu \mathrm{m}}$; at higher $\operatorname{SFRs}\left(\gtrsim 2 M_{\odot} \mathrm{yr}^{-1}\right)$, the excess of SFR $12 \mu \mathrm{m}$ over SFR $22 \mu \mathrm{m}$ is only $\sim 10 \%$. Cluver et al. (2017) and Jarrett et al. (2019) argue that, for normal galaxies (excluding extreme dust-obscured starbursts, which are not a concern in our sample), $\mathrm{SFR}_{12 \mu \mathrm{m}}$ is a better proxy of total infrared luminosity and SFR than SFR $22 \mu \mathrm{m}$, because it is less contaminated by polycyclic aromatic hydrocarbon emission and less dependent on metallicity.

In addition to the far-infrared luminosity, the most common empirical proxies for the SFR in the literature are the $\mathrm{H} \alpha$ luminosity (corrected for extinction), the far-UV plus $24-\mu \mathrm{m}$ luminosity, and the 20-cm radio continuum luminosity; see Kennicutt (1998) for a wellknown review. Boselli et al. (2015) (B15) collected a large database of SFR measurements in nearby spirals, determined with those three methods, as part of their Herschel Reference Survey; some of the SFR values in $\mathrm{B} 15$ came from their own $\mathrm{H} \alpha$ observations and data analysis, others were taken from published literature. When more than one proxy was available for a galaxy, B15 also included the average between the different values. Their SFR catalog includes 64 of our 75 galaxies (Table 1, Column 12).

The list of average SFRs in Boselli et al. (2015) does not include errors. We estimated an approximate error from the dispersion of the SFR values reported for the individual proxies around their respective average SFRs, for all galaxies with more than one SFR
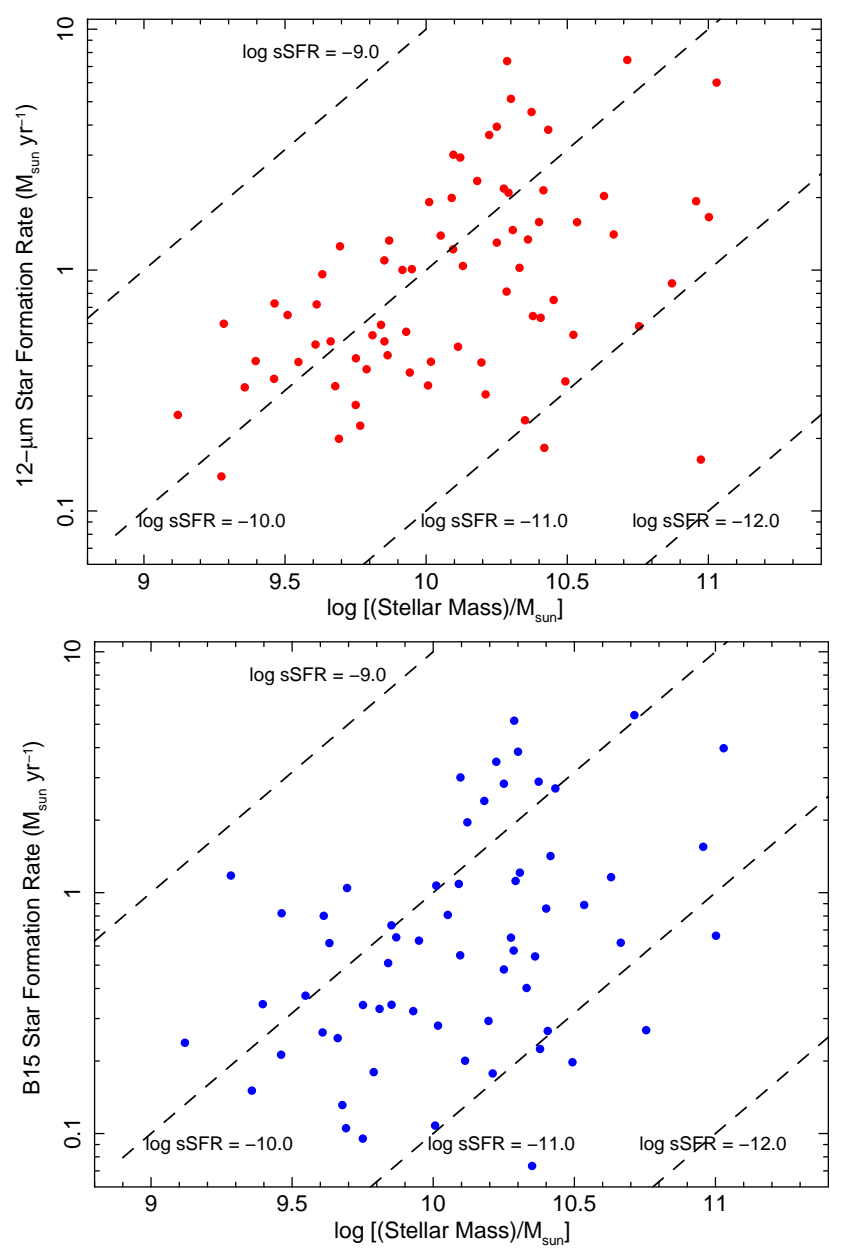

Figure 9. Top panel: $12-\mu \mathrm{m}$ SFR versus $M_{*}$. Loci of constant sSFR are marked with dashed lines. Bottom panel: as in the top panel, for the average B15 SFR.

proxy. The SFRs from the different proxies have an approximately Gaussian distribution around the (normalized) average values, with a standard deviation $\sigma$ of about $20 \%$ of the mean. Thus, we take this standard deviation as an estimate of the $1-\sigma$ error for each B15 average SFR value.

We compared the B15 average values with the WISE values (scaled to the same redshift-independent distance). The B15 values are lower than both the $12-\mu \mathrm{m}$ and $22-\mu \mathrm{m}$ values (Figure 8). For the 64 galaxies that are in common between the WISE catalog and the $\mathrm{B} 15$ catalog, we determine a total $\mathrm{SFR}_{\mathrm{B} 15} \approx 66 M_{\odot} \mathrm{yr}^{-1}$, compared with $\mathrm{SFR}_{22 \mu \mathrm{m}} \approx 81 M_{\odot} \mathrm{yr}^{-1}$ and $\mathrm{SFR}_{12 \mu \mathrm{m}} \approx 98 M_{\odot}$ $\mathrm{yr}^{-1}$. The discrepancy occurs at all values of SFRs, although it is more accentuated at the low end (Figure 7). With the plausible assumption that the same fractional difference would be found in the remaining 11 galaxies, we conclude that the three SFR proxies used by $\mathrm{B} 15$ suggest a total $\mathrm{SFR} \approx 70 M_{\odot} \mathrm{yr}^{-1}$ for our full sample. The large difference cannot be explained with the different choice of IMF (a Salpeter IMF in B15, Salpeter 1955); a conversion to a Kroupa IMF in B15 would reduce rather than increase the amount of stellar mass required to produce the same ionizing flux. An investigation into the different calibrations of the various SFR proxies is beyond the scope of this paper.

Knowing the mass and the SFR, we can then determine the distribution of our sample galaxies in terms of their sSFR. This is 


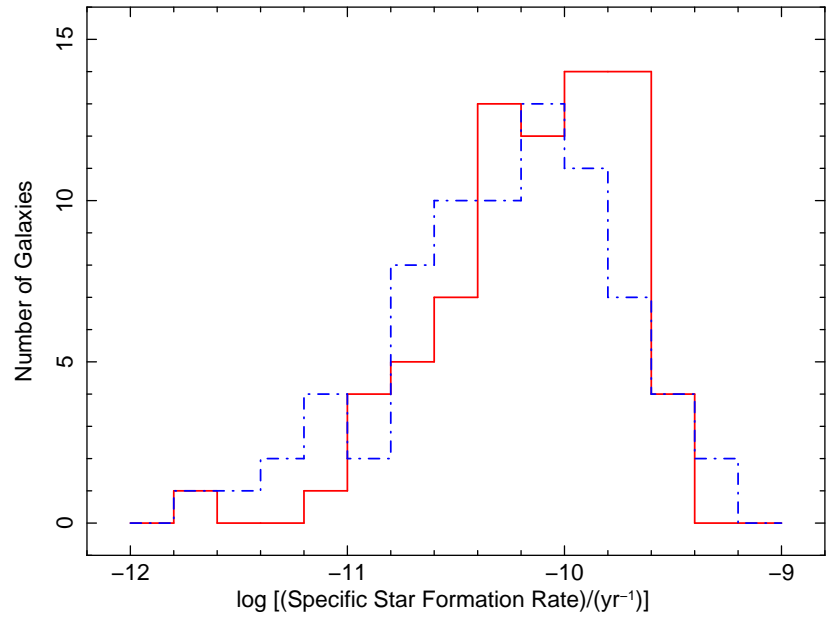

Figure 10. Solid red histogram: sSFR distribution, inferred from the WISE $12-\mu \mathrm{m}$ luminosity. Dash-dotted blue histogram: sSFR distribution from the WISE $22-\mu \mathrm{m}$ luminosity.

a parameter that will be particularly useful later, when we interpret the X-ray luminosity of the galaxies. In the mass versus $\mathrm{SFR}_{12 \mu \mathrm{m}}$ plane (Figures 9,10), about half of our galaxies have sSFR $<-10$, which characterizes the "blue cloud" of star-forming galaxies (compare with Fig. 2 in Lehmer et al. 2019), and the other half can be described as "green valley" galaxies. If we adopt the B15 SFR, about two thirds of the galaxies are in the green valley. The sSFR distribution inferred from $\mathrm{SFR}_{22 \mu \mathrm{m}}$ is of course intermediate between the other two. From this rough classification, we can already speculate that on average, we should find comparable contributions to the X-ray luminosity from galaxies dominated by young HMXBs and those dominated by intermediate-age or old LMXBs (see Fig. 6 in Lehmer et al. 2019 for the predicted transition between the two classes). We investigate this point with our Chandra study.

\section{X-RAY DATA ANALYSIS}

We selected and downloaded from the Chandra archives all the Advanced CCD Imaging Spectrometer (ACIS) observations that included our sample galaxies. That is a total of 110 observations from 2000 February to 2020 March: 106 observations placed the target on the back-illuminated ACIS-S3 chip, and the other 4 on the front-illuminated ACIS-I chips. Fifty-two of the observations were specifically obtained for this project (Proposal 18620568, PI R. Soria, observing cycle 18), with exposure times between 8 and $15 \mathrm{ks}$, for a total exposure time of $551 \mathrm{ks}$; they all placed the target on $\mathrm{S} 3$. The other 58 were gathered from the archives, with various durations, from as short as $1.4 \mathrm{ks}$ to as long as $148 \mathrm{ks}$, adding another 1,413 ks (Table 2, Columns 7-10).

We reprocessed and analysed each dataset with the Chandra Interactive Analysis of Observations (CIAO) software version 4.12 (Fruscione et al. 2006), with calibration database version 4.9.1. In particular, we created new level-2 event files with the ciao tasks chandra_repro. For galaxies with multiple observations, we applied reproject_obs to align and create stacked images. We created individual and stacked images in the soft band $(0.3-1.0 \mathrm{keV})$, medium band (1.0-2.0 keV), hard band (2.0-7.0) and full band (0.3-7.0 keV). We used the Ds9 imaging tool (Joye \& Mandel 2003) for displaying images, defining source and background regions, and performing various other tasks (e.g., smoothing, creating contour plots, align- ing and overplotting X-ray contours over optical images, finding centroids of point-like sources, etc.).

\subsection{Integrated galaxy luminosities}

First, we measured or constrained the total X-ray luminosity of the $\mathrm{X}$-ray binary population of their host galaxies (inside their $D_{25}$ ), including the contribution from faint, unresolved sources. The main hurdles to overcome for such analysis were the choice of suitable background regions (especially in cases when the $D_{25}$ of a galaxy occupied most of the S3 chip) and the potential additional contribution to the X-ray emission from diffuse hot gas and/or from an active nucleus.

We have taken the $B$-band $25 \mathrm{mag} \operatorname{arcsec}^{-2}$ isophotal radii, and thus diameters $\left(D_{25}\right)$, from de Vaucouleurs et al. (1991). We drew corresponding elliptical regions with Ds9, in a format suitable to CIAO analysis. We then ran the CIAO task specextract to create spectra and associated response and ancillary response files. More specifically, because we were extracting spectra of extended regions, we built spatially weighted ancillary response functions (specextract parameter "weight = yes") without a point-source aperture correction ("correctpsf = no"). For each target galaxy, background regions on the same CCD were chosen as large as possible, outside the $D_{25}$ of the galaxy, but we avoided regions of low transmission (caused by molecular contamination) near the edges of the CCD array. Each of the six ACIS-S chips is $8^{\prime} \times 8^{\prime}$, providing enough galaxy-free fieldof-view. As noted previously, the bulk of the galaxy sample had their centre positioned on the S3 chip. We also built response and ancillary response files for the background regions with specextract.

We adopted two methods to subtract the background and model the net galaxy emission. The first method consists of first modelling the background spectrum, scaling that model to the area of the galaxy, then holding this background model component fixed and adding one or more spectral components representing the emission from the galaxy. The second method simply subtracts the background spectrum (suitably scaled) directly from the galaxy-plusbackground spectrum.

In the first method, the background model consists of a celestial $\mathrm{X}$-ray background (which includes unresolved cosmic background from distant AGN, and possible intracluster emission from diffuse hot gas in Virgo) and a particle-induced instrumental background. The celestial X-ray background is modelled as a two-temperature thermal plasma plus a power law with spectral parameter values given by the Athena Wide Field Imager (Meidinger et al. 2020) background preparation document ${ }^{7}$, provided by Arne Rau (Max Planck Institute for Extraterrestrial Physics). We scaled the normalization of this component by the area of the background region on the sky. The instrumental background was modelled as a blackbody and three power-law continua superposed with three Gaussians. All parameters for the black body and the power-law components were allowed to vary in the fitting; however, the Gaussian energies and widths were fixed following the analytical particle background model of Bartalucci et al. (2014), and only their normalizations were allowed to vary. The best-fitting background model was then applied to the source spectrum, with all its parameters frozen, and a global normalization factor scaled by the ratio of the source area to the background area.

Models were fitted to the spectra with the XSPEC (Arnaud 1986)

7 https://www.mpe.mpg.de/ATHENA-WFI/public/resources/background/WFIMPE-ANA-0010_i7.1_Preparation-of-Background-Files.pdf 
package, version 12.11.0. The Cash statistics (Cash 1979) was used as the fit statistics (cstat in XSPEC). An absorbed power-law component was introduced to represent the galactic emission from (resolved and unresolved) point-like sources. Initially, the absorption column density $N_{\mathrm{H}}$ of the galactic power-law component was fixed to the Milky Way line-of-sight value, and the power-law photon index was fixed to $\Gamma=1.7$. In the event of an unacceptable fit, $N_{\mathrm{H}}$ was allowed to vary first, and then, if necessary, the power-law photon index was also thawed.

The second method was a standard, direct subtraction of the background photon spectrum from the source spectrum (i.e., with the source and background spectra loaded in XSPEC as data file and bkg file, respectively). In this case, we did not need to assume any model for the background. We simply modelled the residual emission with an absorbed power-law, with free $N_{\mathrm{H}}$ and $\Gamma$ when we had $\gtrsim 100$ net counts, or a column density fixed to the Galactic line-of-sight value and $\Gamma=1.7$ for galaxies with very few net counts.

We compared the results of the two methods, and found that they were consistent within the uncertainties. In this paper, we have reported the results from the first method. All fits spanned the $0.5-$ $6 \mathrm{keV}$ energy range, because the detected photons are strongly dominated by background above those energies. Observed (model) fluxes and de-absorbed emitted luminosities were then extrapolated and quoted over the $0.5-8 \mathrm{keV}$ range, for consistency with the range chosen by Lehmer et al. (2019), which we will use extensively in comparison to our results.

\subsubsection{Caveat 1: galaxies larger than the S3 chip}

In three cases, a sector at the outskirts of the $D_{25}$ region was outside the detector's field of view (namely, about half of the $D_{25}$ of NGC 4178 and NGC 4579, and about $10 \%$ of NGC 4501). In those cases, the net galaxy flux and luminosity were scaled under the assumption of a uniform surface brightness. The resulting flux may be overestimated by this assumption, because the surface brightness typically decreases towards larger galactic radii. However, given the small number of galaxies affected by this issue, this likely overestimation in flux does not significantly affect our population results; in fact, the uncertainty caused by the rescaling is within the overall model flux uncertainties for each of those galaxies. In another four galaxies with a large apparent size, the central part of the $D_{25}$ falls onto the ACIS-S3 chip but a small sector falls onto either of the two adjoining chips (S2 or S4). In those cases, we defined local background regions that also spanned two chips, with the same fractional area as the source regions. We compared this method with the alternative possibility of computing the galaxy emission only over the fraction of the $D_{25}$ inside the $\mathrm{S} 3$ chip, and then rescaling the total flux as explained above. The two approaches give the same result within the model uncertainties.

\subsubsection{Caveat 2: galaxies with a nuclear X-ray source}

A few of our galaxies contain a bright AGN, with $0.5-8 \mathrm{keV}$ luminosity $\sim 10^{39}-10^{41} \mathrm{erg} \mathrm{s}^{-1}$ : they are classified in the literature as Seyferts (NGC 4388, NGC 4569, NGC 4579, NGC 4639, NGC 4698) or LINERs (NGC 4438, NGC 4450, NGC 4548). Those strong nuclear X-ray sources were immediately excluded from the extraction of the $D_{25}$ spectra of their host galaxies, with the use of "exclude" circular regions around the nucleus. In few cases, bright AGN readout streaks were visible on a chip and were also excluded from our source extraction. In all other cases, when we detected candidate nuclear sources but with an X-ray luminosity $\lesssim 10^{39} \mathrm{erg}$ $\mathrm{s}^{-1}$, we included the nuclear region in the $D_{25}$ source extraction and subsequent model fitting. The emission of those faint, candidate nuclear point sources was extracted and modelled separately, and subtracted from the total $D_{25}$ luminosity of their host galaxies $a$ posteriori. This is because modelling a weak nuclear source on its own and then subtracting its luminosity gives a more accurate result than simply placing an exclusion circle around the nuclear position. In total, 39 of the 75 galaxies (including the 8 mentioned above) have a point-like Chandra source within $\approx 1^{\prime \prime} .5$ of the position of the optical nucleus reported in the literature. A separate paper on the nuclear X-ray properties of those galaxies suspected of hosting an intermediate-mass black hole can be found in Graham et al. (2021), and we have already presented preliminary results for candidate intermediate-mass nuclear BHs (based on archival Chandra data prior to our Large Program observations) in Graham et al. (2019).

\subsubsection{Caveat 3: galaxies with strong thermal-plasma emission}

In a few cases, a simple power law model was statistically unacceptable. Inspection of the fit residuals (and, sometimes, direct inspection of the images) indicated that a soft thermal component was also present in the $D_{25}$ emission, with typical emission features around 1 $\mathrm{keV}, 1.3 \mathrm{keV}$ and $1.8 \mathrm{keV}$; not surprisingly, this happened in galaxies with a high SFR (a beautiful example is NGC 4303). For those galaxies, an additional apec model component was added. The flux from this thermal component was excluded from the galaxy flux reported here, because for this work we are specifically looking at the X-ray binary contribution (both the individually detected sources and the faint, unresolved ones). We are aware that part of the unresolved emission from a galaxy can be in the form of thermal plasma but come from point-like sources; for example, from accretion columns in magnetic Cataclysmic Variables (e.g., Revnivtsev et al. 2007; Li \& Wang 2007; Li et al. 2011; Hong et al. 2016). However, such contribution is negligible compared with the power-law component produced by the X-ray binary population; it is also typically characterized by a temperature $\gtrsim 10 \mathrm{keV}$, so that its spectrum is indistinguishable from a hard power-law component, given the small number of counts for most of our galaxies, and the low Chandra sensitivity above $5 \mathrm{keV}$. It is only the thermal plasma component at temperatures $\sim 0.3-1 \mathrm{keV}$, mostly from gas shock-heated by young SNRs, that (occasionally) stands out in a galaxy spectrum and can be separated from the power-law component. We leave a discussion of the hot gas emission to follow-up work.

\subsubsection{Caveat 4: background AGN projected onto the $D_{25}$}

Area-scaling for background subtraction assumes that the cosmic background emission is uniform between source and background regions. This applies in principle both to unresolved emission and (on a statistical level) to point-like sources (background AGN), leaving aside the issue of cosmic variance. This may not be true for individual galaxies, if their $D_{25}$ ellipse includes one or few bright AGN projected through the galaxy, which may not be exactly balanced by a scaled number of similar AGN in the background region, given the small-number statistics. We will illustrate the expected relative contribution of resolved background AGN in Section 4.2, when we discuss the ULX LF. Here we can anticipate that such a contamination is not a significant problem. We will show that most galaxies have $0.5-8 \mathrm{keV}$ X-ray luminosities $\gtrsim 10^{39}$, and we expect to find a total of only $\sim 10$ AGN projected inside the $D_{25}$ of the 75 sample 
galaxies, at a flux greater than this apparent luminosity (using the median NED distance for each galaxy).

\subsection{Point-source $\mathrm{X}$-ray luminosities}

For this part of the analysis, we used again standard CIAO tasks. In particular, we used wavdetect to identify point-like sources. We then checked and improved the centroid position with dmstat. In a few cases, especially when a source was observed far from the aimpoint, we further refined its position using a 2-D Gaussian fit with the sHERPA package (Freeman et al. 2001). For regions with significant diffuse emission from hot gas, we used the hard band or the $1.5-7 \mathrm{keV}$ band to filter out the thermal plasma emission and identify accreting point-like sources. This includes also the candidate nuclear sources, which will be discussed in a follow-up paper. In this work, we present the results for the off-nuclear ULX population.

We measured absorbed and absorption-corrected fluxes of every moderately bright point-like source (1.e., every source with an observed flux $\gtrsim 10^{-14} \mathrm{erg} \mathrm{cm}^{-2} \mathrm{~s}^{-1}$, corresponding to a luminosity of about a few $\times 10^{38} \mathrm{erg} \mathrm{s}^{-1}$ ) with srcflux, over the $0.5-8 \mathrm{keV}$ band. In doing so, we used the ancillary response function to determine the shape of the point spread function (PSF) at the location of the source (i.e., "PSF Method = arfcorr" in srcflux). As usual for Chandra/ACIS studies, the size of the source extraction region depended on how far from the aimpoint each particular source was. For isolated sources near the aimpoint, the typical source extraction radius was $2^{\prime \prime} .5$, or $2^{\prime \prime} .0$ for sources in more crowded regions. For background subtraction, we defined local background regions at least four times larger that the corresponding source regions.

We made two assumptions in our initial srcflux analysis of the ULX population: a) that the spectrum was a power law with a photon index $\Gamma=1.8$; b) that the absorbing column density $N_{\mathrm{H}}$ was limited to the line-of-sight Galactic value, obtained from HI4PI Collaboration et al. (2016) via NASA's High Energy Astrophysics Science Archive Research Center (HEASARC). The rationale for the first assumption is that $\Gamma \approx 1.8$ is the mean photon index over the $0.5-8 \mathrm{keV}$ band determined from the Swartz et al. (2004)'s Chandra survey of ULXs in nearby galaxies. It is also the slope used for the conversion from count rates to luminosities in the follow-up work of Swartz et al. (2011). The effect of different choices of $\Gamma$ is small, over a plausible range of values. For example, for Cycle-18 observations, with $N_{\mathrm{H}}=3 \times 10^{20} \mathrm{~cm}^{-2}$, the choice of $\Gamma=1.6$ would increase the inferred unabsorbed luminosity by about $6 \%$; the choice of $\Gamma=2.0$ would decrease it by about $3 \%$. The reason for the second assumption (line-of-sight Galactic absorption) is that we wanted to start from a ULX list as simple and model-independent as possible.

In the second step of our point-source analysis (Section 4.2.1), we selected all sources with $\gtrsim 50$ counts. We used specextract to create spectra and associated response and ancillary response files for those sources. We then rebinned the spectra with the FTools (Blackburn 1995) task grppha, to 1 count per bin, and modelled them with XSPEC (Arnaud 1986), using the Cash statistics (Cash 1979). We assumed a power-law model whenever possible, but unlike for our initial srcflux estimate, this time we left the photon index and the intrinsic $N_{\mathrm{H}}$ as free parameters. We used the cflux convolution model in XSPEC to measure absorbed and de-absorbed fluxes in the $0.5-8 \mathrm{keV}$ and $0.3-10 \mathrm{keV}$ bands.
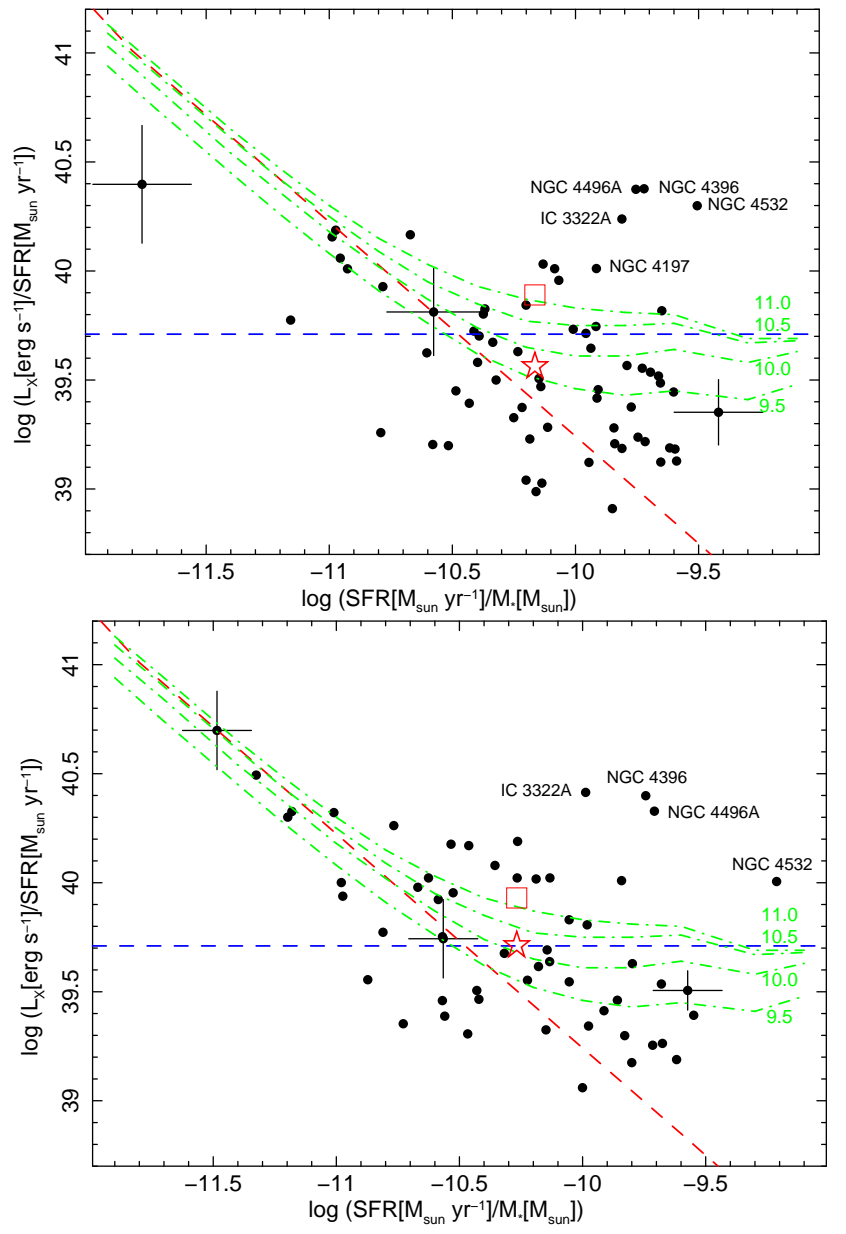

Figure 11. Luminosity of the X-ray binary population $(0.5-8 \mathrm{keV}$ band) of each galaxy, normalized to the SFR of the galaxy, plotted versus the specific SFR. Error bars are omitted for clarity, except for three galaxies in each plot, which visualize representative values of the error bars for low, intermediate and high sSFRs. Errors for all individual galaxies can be derived from the values of $M_{*}$, SFR and $L_{X}$ listed in Table 1, Columns 9-12, and Table 2, Column 2. Top panel: SFR values from the WISE 12$\mu \mathrm{m}$ band; bottom panel: SFR values from B15 (hence, not all 75 galaxies have a datapoint in the bottom panel). The dashed diagonal red line is the theoretical luminosity expected for a pure LMXB population (i.e., scaling with the stellar mass), in the limit of perfect sampling of the LF(Lehmer et al. 2019). The dashed horizontal blue line is the theoretical luminosity for a pure HMXB population, again in the limit of perfect sampling (Lehmer et al. 2019). The red star marks the total value of $L_{X} / S F R$ versus sSFR for all the sample galaxies. The red square is the expected value of $L_{X} / S F R$ versus sSFR for the whole sample (i.e., the sum of the LMXB and HMXB luminosities predicted by Lehmer et al. 2019 at that value of sSFR). The green dashdotted curves are the predicted median values of the observed luminosity, for a population of galaxies of various SFRs and stellar masses, based on the Monte Carlo simulations of Lehmer et al. (2019); the number next to each curve represents the stellar mass along that track. The green curves differ from a simple sum of LMXB plus HMXB luminosity, because of the incomplete sampling of the LF in each galaxy, especially those of lower masses. A few outliers with an exceptionally high $L_{X}$ in relation to their SFR and stellar mass are labelled in both panels and will be discussed individually in follow-up work. 


\section{MAIN RESULTS}

We summarize our main results for the de-absorbed X-ray luminosity of all the sample galaxies, and the fluxes and luminosities of their ULXs, in Table 2.

\subsection{Galaxy luminosities}

The galaxy luminosities (Table 2, Column 2) include the ULXs but exclude the thermal plasma emission and the nuclear sources. This is because here we want to study the relation between the galaxy properties (stellar mass and SFR) and their X-ray binary populations. For galaxies with multiple observations of comparable duration, the luminosity values provided in Column 2 are an average luminosity. For galaxies with multiple observations of very uneven duration, only the deepest observations were used. When a galaxy contains ULXs, the ULX luminosity itself is a significant fraction of the total luminosity (i.e., the total point-source luminosity is usually dominated by the sources at the upper end of the LF).

We stress that for this paper, our main interest is in the population properties of the sample rather than in individual galaxies: specifically, whether the Virgo spirals are dominated by LMXBs or $\mathrm{HMXBs}^{8}$. The combined X-ray luminosity of LMXBs is proportional to the stellar mass (Gilfanov 2004; Lehmer et al. 2010; Boroson et al. 2011; Zhang et al. 2012; Lehmer et al. 2019), although other factors such as population age and specific frequency of globular clusters also affect the correlation (Zhang et al. 2012). The combined HMXB luminosity is proportional to SFR (Grimm et al. 2003; Ranalli et al. 2003; Lehmer et al. 2010; Mineo et al. 2012a; Lehmer et al. 2019). Regardless of the precise value of the normalization constants, we expect a total X-ray luminosity $L_{\mathrm{X}}=$ $\alpha_{\text {LMXB }} M_{*}+\beta_{\text {HMXB }}$ SFR (Lehmer et al. 2010, 2019). Hence, if a population of galaxies are dominated by LMXBs, we expect $L_{\mathrm{X}} / \mathrm{SFR} \propto M_{*} / \mathrm{SFR}=1 / \mathrm{sSFR}$, that is we expect to find them along a line of slope -1 in the $\log \left(L_{X} / \mathrm{SFR}\right)$ versus $\log \mathrm{sSFR}$ plane. Instead, if the galaxies are dominated by HMXBs, we expect them scattered along a horizontal line, because $L_{\mathrm{X}} / \mathrm{SFR} \approx$ constant. In the analysis of Lehmer et al. (2019), the transition from LMXB to HMXB domination occurs at $\mathrm{SSFR} \approx 10^{-10.5} \mathrm{yr}^{-1}$. Their bestfitting relation (which we will use for comparison with our data) is

$L_{\mathrm{X}}=\left(5.1_{-0.9}^{+2.0} \times \mathrm{SFR}+1.8_{-0.3}^{+0.3} \times M_{*, 10}\right) \times 10^{39} \mathrm{erg} \mathrm{s}^{-1}$,

where the SFR is in units of $M_{\odot} \mathrm{yr}^{-1}$ and $M_{*, 10}$ is the stellar mass in units of $10^{10} M_{\odot}$. We have ignored here the small dependence of the SFR coefficient on the metal abundance (Lehmer et al. 2021); we leave this discussion to further work.

Our analysis shows (Figure 11) that there is indeed a (1/sSFR) trend in the distribution of our galaxies at the lower end of the SSFR scale (where we expect LMXBs to dominate). This is more evident when we use the B15 values of SFR (Figure 11, bottom panel): in this case, about $1 / 3$ of the 75 galaxies are in the region of the diagram in which the X-ray luminosity is dominated by LMXBs. This does not mean that $1 / 3$ of the total X-ray luminosity of the Virgo spirals comes from LMXBs, because the galaxies dominated by HMXBs are also the most luminous and the most likely to contain ULXs.

For sSFR $\gtrsim 10^{-10.5} \mathrm{yr}^{-1}$, the galaxy distribution is very scattered and flatter than the $(1 / \mathrm{sSFR})$ trend, but most galaxies fall

8 The possibility of off-centre, wandering intermediate-mass BHs among the point-source population will be discussed in further work. below the median tracks predicted by the Monte Carlo simulations of Lehmer et al. (2019) (despite a few exceptions with higherthan-expected X-ray luminosity, namely NGC 4532, NGC 4396A, NGC 4396 and NGC 4197). We had previously shown (Figure 6) that about two thirds of our sample galaxies have a stellar mass in the range $9.5 \lesssim \log \left(M_{*} / M_{\odot}\right) \lesssim 10.5$. However, the median point-source luminosity of our sample of galaxies in the high sSFR region (Figure 11) falls below the simulated median line for $\log \left(M_{*} / M_{\odot}\right)=-9.5$ in Lehmer et al. (2019). Thus, the X-ray luminosity of several of the most actively star-forming disks appears underestimated, compared with the scaling relations of Lehmer et al. (2019). Those scaling relations were derived from galaxies with discs orientated close to face-on, with low or intermediate inclination; however, our sample contains several galaxies seen at high inclination, with much higher column densities through their discs (particularly in the regions where HMXBs are preferentially located).

On the same $\log \left(L_{X} / \mathrm{SFR}\right)$ versus $\log$ sSFR plane we can also plot the total values from the whole sample, knowing that $L_{\mathrm{X} \text {,tot }} \approx 3.6 \times 10^{41} \mathrm{erg} \mathrm{s}^{-1}$ (from a simple addition of the $0.5-8$ $\mathrm{keV}$ luminosities of each galaxy) and the total SFR $\approx 70-104 M_{\odot}$ $\mathrm{yr}^{-1}$. The total observed value of $L_{\mathrm{X}} / \mathrm{SFR}$ (marked by a red star in the two panels of Figure 11) is lower than predicted by the model of Lehmer et al. (2019) (red squares in the two panels of Figure 11), when we include both the predicted HMXB contribution (dashed blue line) and the predicted LMXB contribution (dashed red line). In particular, the observed luminosity is a factor of 2 lower than predicted if we adopt the $12 \mu \mathrm{m}$ set of SFRs (Figure 11, top panel), or a factor of 1.6 lower if we use the B15 set of SFRs (Figure 11, bottom panel).

At this stage of our analysis, there is no glaring single reason for this discrepancy between inferred and predicted luminosities. We have already hinted at an underestimation of the de-absorbed luminosity from galaxies seen at higher inclination as the most likely cause. We will investigate this possibility further when we fit the individual spectra of the most luminous ULXs and model their luminosity distribution (Sections 4.2.1, 4.3). We will show there that the median absorption column density of the most luminous ULXs is $N_{\mathrm{H}} \approx 3 \times 10^{21} \mathrm{~cm}^{-2}$. If we assume this column density, instead of line-of-sight Galactic absorption, our inferred luminosities would be increased by a factor of $\approx 1.5$ (for observations in Cycle 4 or earlier), a factor $\approx 1.4$ (observations in Cycles 5-14) and a smaller factor for more recent observations ( $\approx 1.2$ for Cycle 18 ). This would bring our total $L_{\mathrm{X}} / S F R$ in line with the prediction from Lehmer et al. (2019), within the errors. (For comparison, Lehmer et al. 2019 found a median intrinsic column density $N_{\mathrm{H}}=2 \times 10^{21}$ for their brighter sources, and applied that value to convert count rates to luminosities for the fainter sources). Even the choice of a higher but constant $N_{\mathrm{H}}$ would be an over-simplification (which is why we avoided it here): galaxies with a high sSFR contain more cold gas and should have an absorption column density above the median value (thus requiring a larger luminosity correction in Figure 11), while galaxies with low sSFR may be better approximated with $N_{\mathrm{H}}$ close to the line-of-sight Galactic value.

Moreover, the relation between $L_{\mathrm{X}}$, SFR and $M_{*}$ may be different for the galaxies in the Virgo sample and those in the Lehmer et al. (2019) sample. There are only 5 Virgo spirals (NGC 4254, NGC 4321, NGC 4450, NGC 4536, NGC 4569) in the sample of 38 galaxies used by Lehmer et al. (2019) to fit their scaling parameters. Even for those 5 galaxies, we cannot directly compare our and their adopted values of $L_{\mathrm{X}}, \mathrm{SFR}$ and $M_{*}$, because Lehmer et al. (2019) use only the inner part of the $D_{25}$ of those 


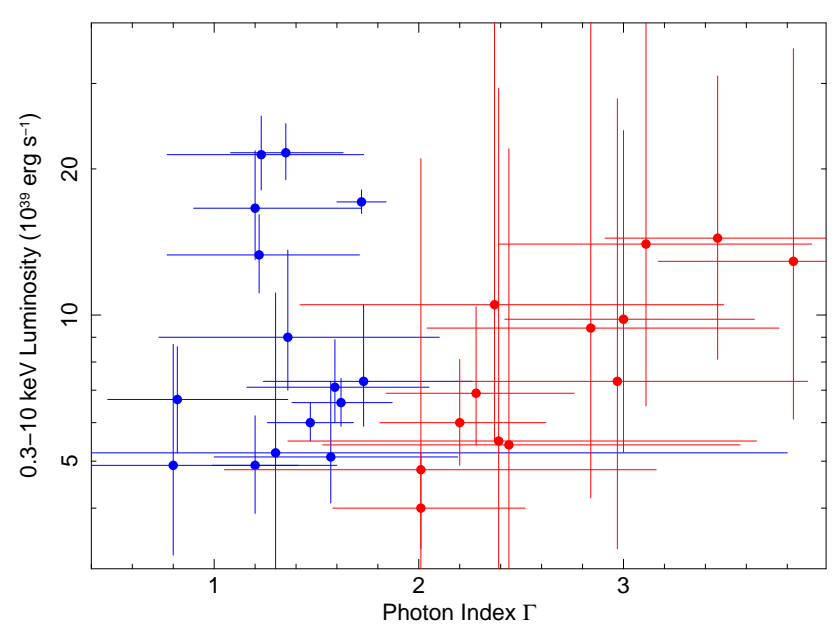

Figure 12. Unabsorbed $0.3-10 \mathrm{keV}$ luminosity of the most luminous ULXs with individual spectral modelling (Table 3) as a function of their spectral hardness (power-law photon index). Blue datapoints correspond to ULXs in the hard ultraluminous state $(\Gamma<2)$; red datapoints correspond to the soft ultraluminous state $(\Gamma>2)$. We omitted from the plot the unusual thermal source (young SN candidate) for which we cannot define a photon index (Section 4.2.2).

spirals (about half of the projected $D_{25}$ area). More specifically, Lehmer et al. (2019) consider only the portion of a galaxy inside the $K_{S}=20 \mathrm{mag}(\operatorname{arcsec})^{-2}$ surface brightness isophote (Jarrett et al. 2003), while we have used the traditional $D_{25}$ definition of galactic sizes. This may introduce a small bias, for example if the outskirts of spiral disks have a lower number of X-ray binaries and lower $L_{X}$ for a given stellar mass or SFR, compared with the denser inner regions. Furthermore, we used different proxies for SFR and different proxies for stellar mass, compared with Lehmer et al. (2019). Finally, we used a different method for determining the total point-source luminosity of a galaxy. We estimated $L_{X}$ from a spectral analysis (Section 3.1). Instead, Lehmer et al. (2019) estimate $L_{X}$ by integrating the $\mathrm{X}$-ray binary luminosity functions. Considering all these methodological differences, the small discrepancy between our empirical estimates and the predicted scaling relations of Lehmer et al. (2019) is not surprising. A more detailed discussion of this issue is beyond the scope of this work.

\subsection{ULX population}

\subsubsection{Spectral modelling for the ULXs}

In the first step of our ULX search (Section 3.2), we had assumed a fixed spectral model (power-law with photon index $\Gamma=1.8$ ) and Galactic line-of-sight column density to each host galaxy (typically, $N_{\mathrm{H}} \approx 2-3 \times 10^{20} \mathrm{~cm}^{-2}$; values from HI4PI Collaboration et al. 2016 and Kalberla et al. 2005). Under those two assumptions, we identified 85 off-nuclear sources that exceed a $0.3-10 \mathrm{keV}$ unabsorbed luminosity of $10^{39} \mathrm{erg} \mathrm{s}^{-1}$ in at least one observation. We report their positions, observed $0.5-8 \mathrm{keV}$ fluxes and unabsorbed $0.3-10 \mathrm{keV}$ luminosities in Table 2, including the fluxes and luminosities in the observations in which the same sources were below the ULX threshold. Table 2 provides a useful working list of interesting point-like sources; however, all luminosity values are likely to be under-estimated, because we have not yet accounted for the loss of X-ray photons due to intrinsic absorption in the host galaxies and around the compact objects. We will now assess the effect of intrinsic absorption, and then improve on our ULX catalogue by using individual spectral modelling at least for the brightest sources.

We obtained reliable fits with free photon index and column density for all the sources with unabsorbed luminosities $\gtrsim 4 \times 10^{39}$ $\mathrm{erg} \mathrm{s}^{-1}$ (Table 3). Instead, the majority of sources with $L_{\mathrm{X}} \sim 1-4$ $\times 10^{39} \mathrm{erg} \mathrm{s}^{-1}$ do not have enough counts below $1 \mathrm{keV}$ to constrain $N_{\mathrm{H}}$ and $\Gamma$ (and therefore the unabsorbed luminosity) independently. This is particularly the case for sources observed in later Chandra cycles, with a much degraded ACIS sensitivity in the soft band. Thus, we limit our spectral analysis to the most luminous ULXs. At the signal-to-noise available for the majority of our sources, an absorbed power-law model provides an adequate fit, with plausible photon indices $1 \lesssim \Gamma \lesssim 3$. The only exception is a ULX (CXOU J122541.70+071338.9) in the disk plane of the perfectly edge-on galaxy IC 3322A, which appears dominated by thermal plasma emission, as we discuss later (Section 4.2.2).

Based on our spectral modelling results, we found at least 25 ULXs that reach a $0.3-10 \mathrm{keV}$ luminosity of $\gtrsim 4 \times 10^{39} \mathrm{erg} \mathrm{s}^{-1}$ at least in one observation (Table 3); 9 of them reached a luminosity of $10^{40} \mathrm{erg} \mathrm{s}^{-1}$. In addition, a 10th ULX exceeded $10^{40} \mathrm{erg} \mathrm{s}^{-1}$, in NGC 4480, if its Tully-Fisher distance of $42 \mathrm{Mpc}$ is correct; however, in that case, NGC 4480 would most likely not be a member of the Virgo cluster. Thus, we omitted that source from subsequent plots and modelling of the cumulative and differential luminosity distributions. For consistency, we also subtracted the SFR and stellar mass of NGC 4480 when we calculated the predicted contribution of HMXBs and LMXBs, and subtracted the projected area of its $D_{25}$ when we calculated the predicted contribution of foreground and background sources. Moreover, Table 3 includes three sources whose identification as stellar-mass accreting compact objects is less clear: the peculiar thermal-plasma source in IC 3322A (Section 4.2.2), and two sources that may be the nuclei of smaller galaxies behind or near NGC 4492 and NGC 4567 (Section 4.2.3).

We examined the hardness distribution as a function of unabsorbed luminosity (Figure 12), for 28 individual observations (as listed in Table 3) of 25 different ULXs above a luminosity of $4 \times 10^{39}$ $\mathrm{erg} \mathrm{s}^{-1}$. We omitted from that plot the four above-mentioned sources with less clear identification, in the $D_{25}$ of IC 3322A, NGC 4480, NGC 4492 and NGC 4567. In 15 out of 28 cases, the sources can be classified in the hard ultraluminous state (using the classification scheme of Sutton et al. 2013), with a photon index $\Gamma<2$ (which means that the spectral luminosity $E L_{E}$ rises with energy, in the Chandra band), and the other 13 are in the soft ultraluminous state, with $\Gamma>2$. The hardness-luminosity distribution (Figure 12) is also consistent with the alternative ULX classification of Pintore et al. (2014), into group 1 (harder) and group 2 (softer) sources. In both ULX classification schemes, the difference between between harder and softer sources is mostly attributed to the scattering optical depth of the super-critical disk outflow along our line of sight. In addition, a harder ULX spectrum may be the signature of a neutron star rather than BH accretor (Pintore et al. 2017; Walton et al. 2018).

In our sample, there is no statistical difference (at least below $10^{40} \mathrm{erg} \mathrm{s}^{-1}$ ) between the luminosity of hard and soft sources, although the four most luminous sources (with $L_{\mathrm{X}}>1.5 \times 10^{40}$ $\mathrm{erg} \mathrm{s}^{-1}$ ) are all in the hard state. The median photon index is $\approx 1.8$, which also justifies our initial assumption of the photon index. The median total absorption column density is $\approx 3.0 \times 10^{21} \mathrm{~cm}^{-2}$, that is a factor of 10 higher than what we had assumed in our preliminary luminosity estimates based on srcflux results (Col. 6 in Table 2).

To correct (at least on a statistical level) the likely underestimation of the preliminary luminosity estimates for the sources that are too faint for individual spectral modelling, we re-estimated 
Table 2. Host-galaxy X-ray binary luminosities and ULX census

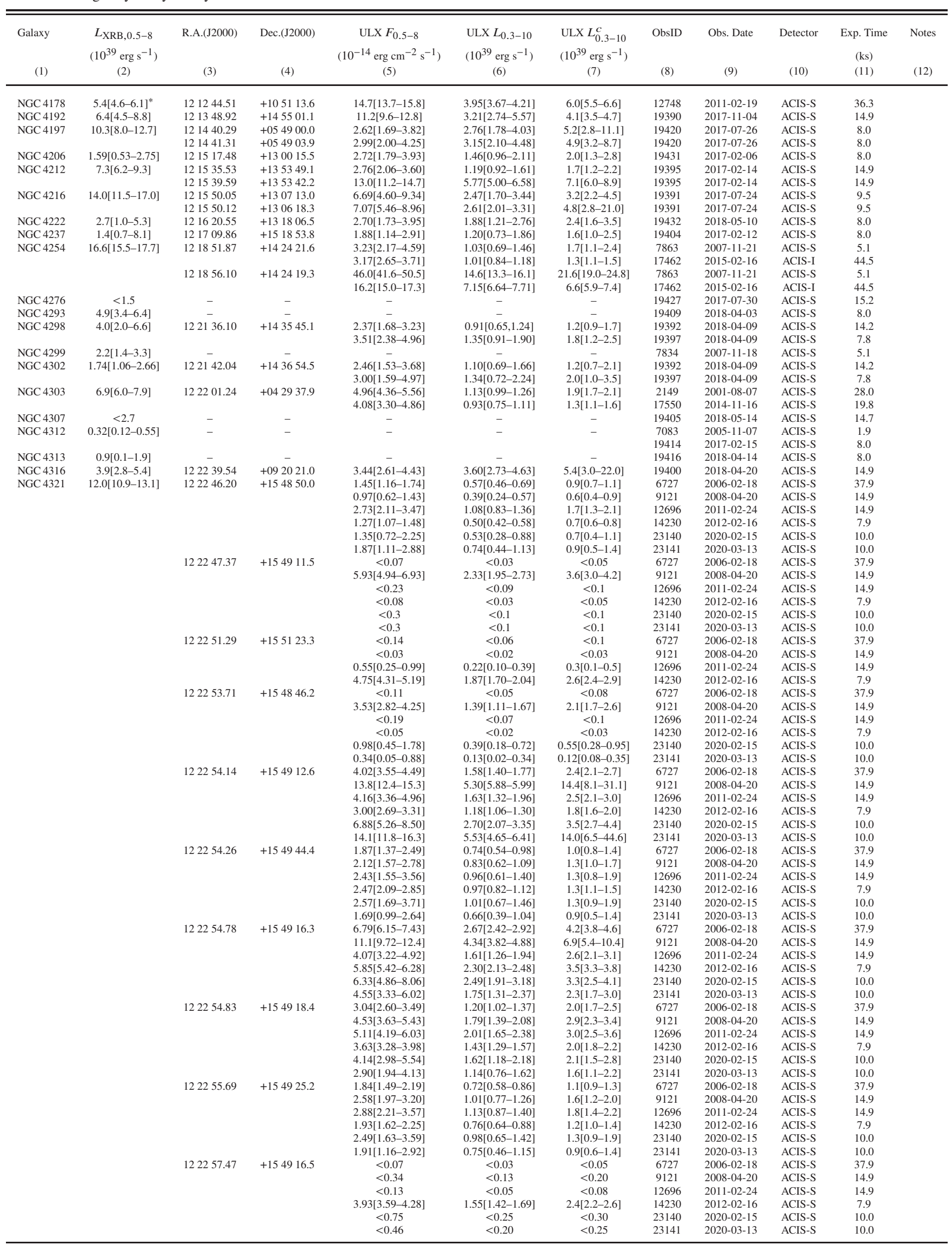


Table 2 - continued Host-galaxy X-ray binary luminosities and ULX census

\begin{tabular}{|c|c|c|c|c|c|c|c|c|c|c|c|}
\hline Galaxy & $\begin{array}{c}L_{\mathrm{XRB}, 0.5-8} \\
\left(10^{39} \mathrm{erg} \mathrm{s}^{-1}\right) \\
(2)\end{array}$ & $\begin{array}{l}\text { R.A.(J2000) } \\
\text { (3) }\end{array}$ & $\begin{array}{l}\text { Dec.(J2000) } \\
\text { (4) }\end{array}$ & $\begin{array}{c}\text { ULX } F_{0.5-8} \\
\left(10^{-14} \mathrm{erg} \mathrm{cm}^{-2} \mathrm{~s}^{-1}\right) \\
(5)\end{array}$ & $\begin{array}{c}\text { ULX } L_{0.3-10} \\
\left(10^{39} \mathrm{erg} \mathrm{s}^{-1}\right) \\
(6)\end{array}$ & $\begin{array}{l}\text { ULX } L_{0.3-10}^{c} \\
\left(10^{39} \mathrm{erg} \mathrm{s}^{-1}\right) \\
(7)\end{array}$ & $\begin{array}{l}\text { ObsID } \\
(8)\end{array}$ & $\begin{array}{l}\text { Obs. Date } \\
\text { (9) }\end{array}$ & $\begin{array}{l}\text { Detector } \\
\text { (10) }\end{array}$ & $\begin{array}{l}\text { Exp. Time } \\
\qquad \begin{array}{l}\text { (ks) } \\
(11)\end{array}\end{array}$ & $\begin{array}{r}\text { Notes } \\
\text { (12) }\end{array}$ \\
\hline NGC 4321 & $12.0[10.9-13.1]$ & 122257.52 & +154847.5 & $<0.08$ & $<0.03$ & $<0.05$ & 6727 & 2006-02-18 & ACIS-S & 37.9 & \\
\hline & & & & $<0.13$ & $<0.05$ & $<0.08$ & 9121 & $2008-04-20$ & ACIS-S & 14.9 & \\
\hline & & & & $<0.13$ & $<0.05$ & $<0.08$ & 12696 & 2011-02-24 & ACIS-S & 14.9 & \\
\hline & & & & $<0.06$ & $<0.03$ & $<0.05$ & 14230 & $2012-02-16$ & ACIS-S & 7.9 & \\
\hline & & & & $4.78[3.55-6.27]$ & $1.88[1.40-2.47]$ & $2.4[1.8-3.2]$ & 23140 & $2020-02-15$ & ACIS-S & 10.0 & \\
\hline & & & & $5.01[3.74-6.54]$ & $1.97[1.47-2.56]$ & $2.5[1.9-3.3]$ & 23141 & 2020-03-13 & ACIS-S & 10.0 & \\
\hline & & 122258.64 & +15 5124.0 & $<0.13$ & $<0.05$ & $<0.08$ & 6727 & $2006-02-18$ & ACIS-S & 37.9 & \\
\hline & & & & $<0.02$ & $<0.01$ & $<0.02$ & 9121 & 2008-04-20 & ACIS-S & 14.9 & \\
\hline & & & & $<0.1$ & $<0.05$ & $<0.08$ & 12696 & $2011-02-24$ & ACIS-S & 14.9 & \\
\hline & & & & $3.28[2.92-3.65]$ & $1.26[1.15-1.44]$ & $1.8[1.6-2.0]$ & 14230 & $2012-02-16$ & ACIS-S & 7.9 & \\
\hline & & & & $<0.38$ & $<0.15$ & $<0.20$ & 23141 & $2020-03-13$ & ACIS-S & 10.0 & \\
\hline & & 122258.68 & +15 4751.9 & $3.20[2.79-3.60]$ & $1.29[1.13-1.46]$ & $2.1[1.8-2.4]$ & 6727 & $2006-02-18$ & ACIS-S & 37.9 & A \\
\hline & & & & $3.70[2.98-4.42]$ & $1.46[1.18-1.74]$ & $2.4[2.0-2.8]$ & 9121 & $2008-04-20$ & ACIS-S & 14.9 & A \\
\hline & & & & $2.40[1.84-3.06]$ & $0.94[0.73-1.20]$ & $1.5[1.2-1.9]$ & 12696 & 2011-02-24 & ACIS-S & 14.9 & A \\
\hline & & & & $3.17[2.86-3.47]$ & $1.25[1.12-1.36]$ & $1.9[1.7-2.1]$ & 14230 & $2012-02-16$ & ACIS-S & 7.9 & A \\
\hline & & & & $1.98[1.21-3.01]$ & $0.78[0.48-1.18]$ & $1.0[0.6-1.5]$ & 23140 & $2020-02-15$ & ACIS-S & 10.0 & A \\
\hline & & & & $1.62[0.93-2.58]$ & $0.64[0.37-1.01]$ & $0.7[0.4-1.2]$ & 23141 & $2020-03-13$ & ACIS-S & 10.0 & A \\
\hline NGC 4330 & $1.62[0.46-3.66]$ & 122313.26 & +112126.0 & $3.63[2.51-5.05]$ & $2.06[1.42-2.86]$ & $2.8[1.9-3.8]$ & 19419 & $2018-04-16$ & ACIS-S & 8.0 & \\
\hline NGC 4343 & $0.5[0.3-1.6]$ & - & - & - & - & - & 4687 & 2005-02-11 & ACIS-S & 38.3 & \\
\hline & & & & & & & 7129 & $2006-07-16$ & ACIS-S & 4.6 & \\
\hline & & & & & & & 12955 & $2011-02-17$ & ACIS-S & 72.8 & \\
\hline NGC 4356 & $<1.0$ & - & - & - & - & - & 19435 & $2017-04-16$ & ACIS-S & 14.9 & \\
\hline NGC 4380 & $2.7[2.0-4.0]$ & 122522.21 & +100103.4 & $2.92[2.16-3.83]$ & $1.65[1.21-2.16]$ & $2.2[1.6-2.9]$ & 19406 & $2018-05-11$ & ACIS-S & 14.7 & \\
\hline IC $3322 \mathrm{~A}$ & $6.4[4.6-8.5]$ & 122541.70 & +071338.9 & $5.46[4.26-6.67]$ & $5.23[4.01-6.39]$ & $62[23-132]$ & 19401 & 2018-04-24 & ACIS-S & 13.9 & \\
\hline & & 122542.25 & +071319.8 & $1.19[0.71-1.84]$ & $1.15[0.68-1.78]$ & $1.6[0.9-2.4]$ & 19401 & 2018-04-24 & ACIS-S & 13.9 & \\
\hline NGC 4388 & $4.6[3.7-5.6]$ & 122538.98 & +124000.8 & $2.89[2.37-3.41]$ & $(1.45[1.19-1.71])$ & $(1.7[1.3-2.2])$ & 1619 & 2001-06-08 & ACIS-S & 20.0 & B \\
\hline & & & & $<0.073$ & $(<0.037)$ & $(<0.05)$ & 12291 & 2011-12-07 & ACIS-S & 27.6 & B \\
\hline & & 122539.14 & +124009.4 & $4.05[3.44-4.67]$ & $2.03[1.72-2.35]$ & $3.4[2.9-3.9]$ & 1619 & $2001-06-08$ & ACIS-S & 20.0 & \\
\hline & & & & $1.48[1.13-1.88]$ & $0.75[0.57-0.94]$ & $1.1[0.8-1.4]$ & 12291 & 2011-12-07 & ACIS-S & 27.6 & \\
\hline & & 122554.06 & +124004.2 & $1.67[1.29-2.07]$ & $0.86[0.66-1.06]$ & $1.5[1.1-1.8]$ & 1619 & 2001-06-08 & ACIS-S & 20.0 & $\mathrm{C}$ \\
\hline & & & & $2.98[2.48-3.48]$ & $1.53[1.28-1.79]$ & $2.4[2.0-2.8]$ & 12291 & 2011-12-07 & ACIS-S & 27.6 & $\mathrm{C}$ \\
\hline NGC 4390 & $0.3[0.1-1.1]$ & - & - & - & - & - & 19425 & 2018-04-14 & ACIS-S & 15.4 & \\
\hline IC 3322 & $0.6[0.16-1.6]$ & - & - & - & - & - & 19424 & $2018-04-22$ & ACIS-S & 14.9 & \\
\hline NGC 4394 & $1.0[0.5-2.0]$ & - & - & - & - & - & 7864 & $2007-11-11$ & ACIS-S & 5.1 & \\
\hline NGC 4396 & $3.8[2.9-4.7]$ & 122601.54 & +153941.4 & $4.17[2.97-5.66]$ & $1.08[0.77-1.46]$ & $1.5[1.1-2.0]$ & 19417 & $2018-05-25$ & ACIS-S & 8.1 & \\
\hline & & 122601.81 & +153941.8 & $12.3[10.1-14.6]$ & $3.18[2.58-3.76]$ & $9.4[4.2-40.4]$ & 19417 & $2018-05-25$ & ACIS-S & 8.1 & \\
\hline NGC 4402 & $2.3[1.7-3.4]$ & - & - & - & - & - & 15149 & 2013-07-24 & ACIS-S & 148.1 & \\
\hline NGC 4405 & $1.0[0.6-2.2]$ & - & - & - & - & - & 19410 & 2018-04-09 & ACIS-S & 8.0 & \\
\hline NGC $4411 \mathrm{~A}$ & $1.49[0.88-2.25]$ & 122627.86 & +085145.03 & $3.34[2.29-4.67]$ & $1.43[0.98-2.00]$ & $2.3[1.6-3.2]$ & 7837 & $2007-11-13$ & ACIS-S & 5.1 & \\
\hline & & 122631.62 & +085228.1 & $3.84[2.55-5.50]$ & $1.63[1.09-2.34]$ & $2.5[1.7-3.6]$ & 7840 & $2007-02-13$ & ACIS-S & 4.9 & \\
\hline & & & & $1.06[0.51-1.90]$ & $0.45[0.21-0.81]$ & $0.7[0.3-1.3]$ & 7837 & $2007-11-13$ & ACIS-S & 5.1 & \\
\hline & & & & $3.61[2.49-5.02]$ & $1.54[1.06-2.13]$ & $1.9[1.3-2.6]$ & 19429 & 2017-02-09 & ACIS-S & 9.9 & \\
\hline NGC 4412 & $3.6[1.8-6.6]$ & 122635.16 & +035815.0 & $0.85[0.36-1.62]$ & $1.67[0.70-3.20]$ & $2.4[1.1-4.5]$ & 19418 & $2017-11-23$ & ACIS-S & 8.0 & D \\
\hline NGC 4411B & $2.4[1.7-3.6]$ & 122648.76 & +085338.1 & $1.68[0.96-2.70]$ & $1.27[0.72-2.05]$ & $2.0[1.1-3.2]$ & 7840 & $2007-02-13$ & ACIS-S & 4.9 & \\
\hline & & & & $2.84[1.98-3.91]$ & $2.12[1.50-2.01]$ & $2.8[2.0-3.9]$ & 19429 & 2017-02-09 & ACIS-S & 9.9 & \\
\hline & & 122649.48 & +08 5218.9 & $2.83[1.85-4.10]$ & $2.14[1.40-3.10]$ & $3.4[2.2-4.8]$ & 7840 & $2007-02-13$ & ACIS-S & 4.9 & \\
\hline & & & & $3.80[2.79-5.03]$ & $2.88[2.08-3.74]$ & $5.5[2.8-29.3]$ & 19429 & 2017-02-09 & ACIS-S & 9.9 & \\
\hline NGC 4407 & $2.7[0.9-4.9]$ & _- & _- & - & $2.001-1$ & - & 19434 & 2018-05-10 & ACIS-S & 7.8 & \\
\hline NGC 4416 & $<1$ & _- & _- & _- & _- & _- & 19436 & 2018-05-06 & ACIS-S & 7.8 & \\
\hline NGC 4419 & $4.3[3.2-6.5]$ & - & - & - & - & - & 19394 & $2016-12-24$ & ACIS-S & 9.9 & \\
\hline NGC 4424 & $0.9[0.5-1.5]$ & - & - & - & - & - & 19408 & 2017-04-17 & ACIS-S & 14.9 & \\
\hline NGC 4429 & $4.1[2.0-6.3]$ & - & - & - & - & - & 19430 & 2018-05-10 & ACIS-S & 7.8 & \\
\hline NGC 4430 & $2.6[1.9-3.7]$ & 122727.09 & +061553.9 & $5.53[4.04-7.35]$ & $2.10[1.53-2.79]$ & $2.9[2.1-3.8]$ & 19426 & 2018-05-05 & ACIS-S & 7.8 & \\
\hline NGC 4438 & $3.9[2.9-5.1]$ & - & - & - & - & - & 2883 & $2002-01-29$ & ACIS-S & 25.1 & \\
\hline & & & & & & & 8042 & $2008-02-11$ & ACIS-S & 4.9 & \\
\hline & & & & & & & 21376 & $2020-03-20$ & ACIS-S & 29.7 & \\
\hline & & & & & & & 23189 & $2020-03-20$ & ACIS-S & 19.8 & \\
\hline & & & & & & & 23037 & $2020-03-27$ & ACIS-S & 19.8 & \\
\hline & & & & & & & 23200 & $2020-03-28$ & ACIS-S & 25.7 & \\
\hline NGC 4445 & $1.0[0.4-2.2]$ & 122816.38 & +092608.4 & $2.86[2.12-3.76]$ & $1.48[1.09-1.94]$ & $1.9[1.4-2.5]$ & 19433 & $2018-05-13$ & ACIS-S & 14.9 & \\
\hline NGC 4450 & $8.4[5.6-11.3]$ & 122831.37 & +170426.8 & $3.68[2.58-5.04]$ & $1.30[0.92-1.79]$ & $1.8[1.3-2.5]$ & 19399 & 2017-04-06 & ACIS-S & 8.0 & \\
\hline NGC 4451 & $1.3[0.7-2.1]$ & - & - & 2000 & - & - & 19412 & 2018-05-13 & ACIS-S & 14.7 & \\
\hline IC 3392 & $<0.6$ & - & - & - & - & - & 19421 & 2018-04-11 & ACIS-S & 7.6 & \\
\hline NGC 4457 & $0.7[0.4-0.9]$ & - & - & - & - & - & 3150 & $2002-12-03$ & ACIS-S & 38.9 & \\
\hline NGC 4469 & $1.1[0.6-1.6]$ & - & - & - & - & - & 19415 & $2018-06-23$ & ACIS-S & 14.9 & \\
\hline NGC 4470 & $1.0[0.8-1.2]$ & 122938.03 & +074932.3 & $3.11[2.46-3.78]$ & $1.30[1.02-1.58]$ & $2.0[1.6-2.4]$ & 12978 & $2010-11-20$ & ACIS-S & 19.8 & \\
\hline & & & & $4.23[3.12-5.35]$ & $1.74[1.28-2.20]$ & $2.7[1.9-3.5]$ & 15756 & 2014-04-16 & ACIS-I & 32.1 & \\
\hline & & & & $2.57[1.84-3.44]$ & $1.06[0.76-1.42]$ & $1.5[1.1-1.9]$ & 15760 & $2014-04-26$ & ACIS-I & 29.4 & \\
\hline & & & & $1.86[1.35-2.47]$ & $0.76[0.56-1.02]$ & $1.1[0.8-1.5]$ & 16260 & 2014-08-04 & ACIS-S & 24.7 & \\
\hline & & & & $2.10[1.57-2.71]$ & $0.87[0.65-1.11]$ & $1.2[0.9-1.6]$ & 16261 & $2015-02-24$ & ACIS-S & 22.8 & \\
\hline & & & & $2.27[1.51-3.24]$ & $0.94[0.62-1.34]$ & $1.4[1.0-1.8]$ & 16262 & 2016-04-30T & ACIS-S & 24.7 & \\
\hline & & & & $2.78[2.11-3.56]$ & $1.15[0.87-1.46]$ & $1.4[1.1-1.8]$ & 21647 & 2019-04-17 & ACIS-S & 29.7 & \\
\hline NGC 4480 & $11.0[6.8-16.6]$ & 123027.86 & +04 1459.9 & $1.14[5.89-1.96]$ & $3.08[1.60-5.30]$ & $4.3[2.2-7.4]$ & 19423 & $2016-12-06$ & ACIS-S & 8.0 & $\mathrm{D}$ \\
\hline & & 123028.14 & +041440.9 & $4.02[2.89-5.42]$ & $10.9[7.9-14.7]$ & $13.0[8.3-35.4]$ & 19423 & $2016-12-06$ & ACIS-S & 8.0 & $\mathrm{D}$ \\
\hline NGC 4492 & $3.7[2.6-5.2]$ & 123057.83 & +08 0435.2 & $0.83[0.33-1.65]$ & $0.46[0.18-0.93]$ & $0.6[0.2-1.3]$ & 7845 & $2007-02-22$ & ACIS-S & 4.9 & E \\
\hline & & & & $7.01[6.05-7.99]$ & $3.94[3.40-4.50]$ & $8.3[6.2-18.1]$ & 15759 & $2014-04-25$ & ACIS-I & 29.7 & $\mathrm{E}$ \\
\hline & & 123058.88 & +08 0452.2 & $4.18[2.98-5.67]$ & $2.35[1.68-3.18]$ & $3.7[2.6-5.0]$ & 7845 & $2007-02-22$ & ACIS-S & 4.9 & \\
\hline & & & & $2.54[1.94-3.15]$ & $1.43[1.10-1.77]$ & $2.0[1.5-2.4]$ & 15759 & $2014-04-25$ & ACIS-I & 29.7 & \\
\hline NGC 4496A & $17.1[12.0-23.3]$ & 123138.08 & +035642.2 & $35.5[29.3-41.8]$ & $13.4[10.9-15.8]$ & $16.6[13.0-21.8]$ & 16995 & $2015-07-26$ & ACIS-S & 2.7 & \\
\hline NGC 4498 & $0.9[0.5-1.4]$ & - & - & - & - & - & 19422 & 2018-04-07 & ACIS-S & 8.1 & \\
\hline NGC 4501 & $12.7[9.5-15.9]^{*}$ & 123200.39 & +142442.4 & $8.05[7.09-9.02]$ & $3.61[3.18-4.04]$ & $12.9[6.1-35.4]$ & 2922 & 2002-12-09 & ACIS-S & $\begin{array}{l}0.1 \\
17.9\end{array}$ & \\
\hline & & 123200.94 & +142502.6 & $9.16[8.15-10.2]$ & $4.10[3.64-4.59]$ & $6.0[4.9-8.1]$ & 2922 & $2002-12-09$ & ACIS-S & 17.9 & \\
\hline & & 123206.19 & +142321.5 & $2.36[1.76-3.05]$ & $1.06[0.79-1.36]$ & $1.6[1.2-2.1]$ & 2922 & 2002-12-09 & ACIS-S & 17.9 & \\
\hline NGC 4519 & $1.9[0.9-2.9]$ & - & - & - & _ & - & 19411 & 2018-05-05 & ACIS-S & 8.5 & \\
\hline NGC 4522 & $<0.9$ & - & - & - & - & - & 19428 & 2018-05-05 & ACIS-S & 7.8 & \\
\hline
\end{tabular}


Table 2 - continued Host-galaxy X-ray binary luminosities and ULX census

\begin{tabular}{|c|c|c|c|c|c|c|c|c|c|c|c|}
\hline Galaxy & $\begin{array}{c}L_{\mathrm{XRB}, 0.5-8} \\
\left(10^{39} \mathrm{erg} \mathrm{s}^{-1}\right) \\
(2)\end{array}$ & R.A.(J2000) & Dec.(J2000) & $\begin{array}{c}\text { ULX } F_{0.5-8} \\
\left(10^{-14} \mathrm{erg} \mathrm{cm}^{-2} \mathrm{~s}^{-1}\right) \\
(5)\end{array}$ & $\begin{array}{c}\text { ULX } L_{0.3-10} \\
\left(10^{39} \mathrm{erg} \mathrm{s}^{-1}\right) \\
(6)\end{array}$ & $\begin{array}{c}\text { ULX } L_{0.3-10}^{c} \\
\left(10^{39} \mathrm{erg} \mathrm{s}^{-1}\right) \\
\text { (7) }\end{array}$ & $\begin{array}{l}\text { ObsID } \\
(8)\end{array}$ & $\begin{array}{l}\text { Obs. Date } \\
\text { (9) }\end{array}$ & Detector & $\begin{array}{l}\text { Exp. Time } \\
\text { (ks) } \\
(11)\end{array}$ & $\begin{array}{l}\text { Notes } \\
\text { (12) }\end{array}$ \\
\hline \multirow[t]{8}{*}{ NGC 4527} & $7.5[5.6-9.6]$ & 123410.94 & +023925.2 & $24.7[21.6-27.9]$ & $7.4[6.5-8.4]$ & $21.4[18.1-25.7]$ & 4017 & 2003-03-09 & ACIS-S & 4.9 & \\
\hline & & & & $0.43[0.13-0.95]$ & $0.13[0.04-0.29]$ & $0.18[0.06-0.40]$ & 19386 & 2016-12-18 & ACIS-S & 9.9 & \\
\hline & & 123411.43 & +023928.9 & $18.0[15.4-20.7]$ & $5.4[4.6-6.2]$ & $13.3[11.1-16.1]$ & 4017 & 2003-03-09 & ACIS-S & 4.9 & \\
\hline & & & & $3.11[2.20-4.23]$ & $0.94[0.66-1.27]$ & $1.3[0.9-1.8]$ & 19386 & $2016-12-18$ & ACIS-S & 9.9 & \\
\hline & & 123411.76 & +023957.7 & $<0.34$ & $<0.10$ & $<0.17$ & 4017 & 2003-03-09 & ACIS-S & 4.9 & \\
\hline & & & & $3.94[2.91-5.18]$ & $1.18[0.87-1.56]$ & $1.6[1.2-2.1]$ & 19386 & $2016-12-18$ & ACIS-S & 9.9 & \\
\hline & & 123414.87 & +024005.3 & $<0.34$ & $<0.10$ & $<0.17$ & 4017 & 2003-03-09 & ACIS-S & 4.9 & \\
\hline & & & & $5.28[4.08-6.72]$ & $1.58[1.22-2.01]$ & $2.2[1.7-2.7]$ & 19386 & $2016-12-18$ & ACIS-S & 9.9 & \\
\hline \multirow[t]{2}{*}{ NGC 4532} & $11.9[10.6-13.4]$ & 123418.89 & +062815.4 & $20.0[17.1-23.0]$ & $5.13[4.38-5.91]$ & $7.3[5.9-10.5]$ & 19407 & 2018-05-15 & ACIS-S & 8.0 & \\
\hline & & 123420.34 & +062737.5 & $11.4[9.22-13.7]$ & $2.93[2.36-3.51]$ & $7.3[3.3-27.9]$ & 19407 & 2018-05-15 & ACIS-S & 8.0 & \\
\hline NGC 4535 & $3.1[1.2-7.5]$ & 123429.94 & +08 1035.4 & $7.68[6.35-9.02]$ & $(2.98[2.47-3.50])$ & $(4.0[3.3-4.7])$ & 19388 & $2017-04-21$ & ACIS-S & 14.9 & $\mathrm{~F}$ \\
\hline \multirow{6}{*}{ NGC 4536} & $12.0[10.8-13.1]$ & 123426.16 & +021122.3 & $9.31[7.89-10.7]$ & $3.15[2.67-3.64]$ & $5.1[4.1-7.3]$ & 19387 & $2017-07-01$ & ACIS-S & 14.9 & \\
\hline & & 123426.83 & +021118.8 & $3.08[2.30-4.02]$ & $1.08[0.80-1.40]$ & $1.4[1.0-1.9]$ & 19387 & $2017-07-01$ & ACIS-S & 14.9 & \\
\hline & & 123431.81 & +021121.9 & $3.99[3.05-4.93]$ & $1.35[1.04-1.66]$ & $1.8[1.4-2.3]$ & 19387 & 2017-07-01 & ACIS-S & 14.9 & \\
\hline & & 123432.56 & +021054.5 & $7.89[6.57-9.21]$ & $2.67[2.23-3.12]$ & $3.7[3.1-4.3]$ & 19387 & 2017-07-01 & ACIS-S & 14.9 & \\
\hline & & 123436.93 & +020851.4 & $13.1[11.3-15.0]$ & $4.44[3.80-5.06]$ & $9.8[5.2-24.0]$ & 19387 & 2017-07-01 & ACIS-S & 14.9 & \\
\hline & & 123438.81 & +021028.5 & $5.57[4.41-6.74]$ & $1.89[1.49-2.28]$ & $2.5[2.0-3.0]$ & 19387 & 2017-07-01 & ACIS-S & 14.9 & \\
\hline NGC 4548 & $2.2[1.7-3.4]$ & 123531.33 & +143048.8 & $4.72[3.37-6.37]$ & $1.77[1.25-2.38]$ & $2.4[1.7-3.2]$ & 19402 & 2018-04-11 & ACIS-S & 7.6 & \\
\hline NGC 4567 & $9.6[7.2-13.4]$ & 123633.75 & +111610.6 & $4.96[3.62-6.59]$ & $3.87[2.83-5.14]$ & $(4.9[3.4-7.4])$ & 19396 & $2018-05-10$ & ACIS-S & 7.8 & $\mathrm{E}$ \\
\hline \multirow[t]{8}{*}{ NGC 4568} & $12.1[9.0-15.1]$ & 123632.32 & +111306.3 & $3.18[2.10-4.54]$ & $1.44[0.95-2.05]$ & $1.6[1.1-2.1]$ & 19389 & 2018-03-01 & ACIS-S & 13.7 & \\
\hline & & & & $3.41[1.74-5.82]$ & $1.58[0.81-2.72]$ & $2.0[1.0-3.5]$ & 19396 & 2018-05-10 & ACIS-S & 7.8 & \\
\hline & & 123633.04 & +11 1405.6 & $12.1[9.72-14.4]$ & $5.43[4.38-6.50]$ & $6.7[5.2-8.6]$ & 19389 & 2018-03-01 & ACIS-S & 13.7 & \\
\hline & & & & $17.3[13.6-20.8]$ & $7.81[6.14-9.43]$ & $9.0[7.0-13.6]$ & 19396 & 2018-05-10 & ACIS-S & 7.8 & \\
\hline & & 123633.22 & +11 1437.1 & $9.87[7.82-11.9]$ & $4.45[3.43-5.36]$ & $4.9[3.9-6.2]$ & 19389 & 2018-03-01 & ACIS-S & 13.7 & \\
\hline & & & & $1.90[0.92-3.42]$ & $0.86[0.42-1.55]$ & $1.6[1.0-2.3]$ & 19396 & 2018-05-10 & ACIS-S & 7.8 & \\
\hline & & 123634.48 & +111440.2 & $2.20[1.54-3.03]$ & $1.03[0.72-1.14]$ & $1.4[0.9-1.9]$ & 19389 & 2018-03-01 & ACIS-S & 13.7 & \\
\hline & & & & $1.86[1.08-2.93]$ & $0.87[0.51-1.37]$ & $1.2[0.7-1.8]$ & 19396 & 2018-05-10 & ACIS-S & 7.8 & \\
\hline \multirow[t]{2}{*}{ NGC 4569} & $7.4[6.3-8.5]$ & 123653.70 & +131154.0 & $4.93[3.03-7.50]$ & $1.00[0.61-1.52]$ & $1.8[1.1-2.7]$ & 405 & $2000-02-17$ & ACIS-S & 1.7 & \\
\hline & & & & $2.53[2.16-2.89]$ & $0.51[0.44-0.59]$ & $0.82[0.70-0.94]$ & 5911 & $2005-11-13$ & ACIS-S & 39.1 & \\
\hline NGC 4571 & $2.0[0.3-5.6]$ & _- & _- & - & - & - & 7858 & $2008-02-14$ & ACIS-S & 4.7 & \\
\hline \multirow[t]{3}{*}{ NGC 4579} & $28.3[26.5-30.2]^{*}$ & 123740.31 & +114727.8 & $28.9[27.8-30.1]$ & $12.6[12.1-13.1]$ & $17.1[16.2-18.1]$ & 807 & $2000-05-02$ & ACIS-S & 33.9 & \\
\hline & & 123743.21 & +114901.5 & $3.34[2.53-4.15]$ & $1.41[1.07-1.82]$ & $2.4[2.1-2.7]$ & 807 & $2000-05-02$ & ACIS-S & 33.9 & \\
\hline & & 123753.88 & +115020.4 & $9.47[8.14-10.8]$ & $4.12[3.53-4.74]$ & $4.0[2.8-4.7]$ & 807 & $2000-05-02$ & ACIS-S & 33.9 & \\
\hline NGC 4580 & $1.2[0.6-2.1]$ & - & - & - & - & - & 10413 & $2017-12-01$ & ACIS-S & 8.0 & \\
\hline NGC 4606 & $1.2[0.4-2.1]$ & - & - & - & - & - & 19437 & $2017-04-22$ & ACIS-S & 7.7 & \\
\hline \multirow[t]{2}{*}{ NGC 4607} & $3.2[2.4-4.7]$ & 124112.61 & +115350.2 & $1.49[0.75-2.60]$ & $0.89[0.44-1.54]$ & $1.2[0.6-2.2]$ & 19437 & 2017-04-22 & ACIS-S & 7.7 & \\
\hline & & & & $7.98[6.12-9.85]$ & $4.74[3.64-5.87]$ & $10.5[5.5-50.6]$ & 19403 & 2018-05-09 & ACIS-S & 8.0 & \\
\hline \multirow[t]{2}{*}{ NGC 4639} & $5.2[3.8-7.8]$ & 124251.20 & +131440.3 & $3.52[1.82-6.05]$ & $2.58[1.33-4.45]$ & $4.8[2.5-16.8]$ & 408 & $2000-02-05$ & ACIS-S & 1.4 & \\
\hline & & & & $4.70[3.66-5.75]$ & $3.45[2.68-4.22]$ & $4.5[3.5-5.5]$ & 19398 & 2018-04-14 & ACIS-S & 14.7 & \\
\hline \multirow{6}{*}{ NGC 4647} & $6.9[5.1-8.8]$ & - & - & - & - & - & 785 & $2000-04-20$ & ACIS-S & 38.1 & \\
\hline & & & & & & & 8182 & $2007-01-30$ & ACIS-S & 52.3 & \\
\hline & & & & & & & 8507 & 2007-02-01 & ACIS-S & 17.5 & \\
\hline & & & & & & & 12975 & 2011-08-08 & ACIS-S & 84.9 & \\
\hline & & & & & & & 12976 & 2011-02-24 & ACIS-S & 101.0 & \\
\hline & & & & & & & 14328 & 2011-08-12 & ACIS-S & 14.0 & \\
\hline \multirow[t]{2}{*}{ NGC 4654} & $3.9[3.0-4.9]$ & _- & _- & - & _- & _- & 3325 & $2002-05-03$ & ACIS-S & 4.9 & \\
\hline & & & & & & & 19393 & $2016-12-14$ & ACIS-S & 9.9 & \\
\hline NGC 4689 & $5.7[4.0-7.4]$ & 124747.71 & +134617.3 & $7.93[6.09-9.79]$ & $2.99[2.29-3.68]$ & $4.7[3.6-5.8]$ & 7865 & 2007-05-07 & ACIS-S & 4.9 & \\
\hline NGC 4698 & $9.0[6.8-11.5]$ & 124825.87 & +083020.8 & $14.7[13.8-15.7]$ & NA & NA & 3008 & $2002-06-16$ & ACIS-S & 29.7 & G \\
\hline NGC 4713 & $2.0[1.5-3.4]$ & - & - & - & - & - & 4019 & $2003-01-28$ & ACIS-S & 4.9 & \\
\hline
\end{tabular}

Col. (2): total unabsorbed $0.5-8 \mathrm{keV}$ luminosity of the point-source (X-ray binaries) population (resolved plus unresolved) in each galaxy, estimated with a power-law model fit. The range of values in brackets represents the $90 \%$ confidence range. When a galaxy was observed multiple times, the luminosity was determined from a stack of all the observations (combined with the epicspeccombine task). An asterisk (on 3 galaxies) denotes that they are larger than the detector field of view in one direction. See Section 3.1 for details. Cols. (3),(4): coordinates of each ULX, defined as each source that exceeded a 0.3-10 keV luminosity of $10^{39} \mathrm{erg} \mathrm{s}^{-1}$ in at least one of the observations. Col. (5): absorbed $0.5-8 \mathrm{keV}$ flux (with $90 \%$ confidence range) of each ULX in each observation, measured with srcflux; here, we assumed a power-law model, with fixed photon index $\Gamma=1.8$ and only Galactic line-of-sight column density. Col. (6): unabsorbed $0.3-10 \mathrm{keV}$ luminosity (with $90 \%$ confidence range) of each ULX in each observation (including those in which they were in a faint state), assuming $\Gamma=1.8$ and Galactic line-of-sight column density. The conversion from fluxes to luminosities is based on the median redshift-independent NED distances. The error range in the luminosity includes only the flux measurement uncertainty, not the systematic uncertainty in the distance to each galaxy. Col. (7): "corrected" $0.3-10 \mathrm{keV}$ ULX luminosity (with $90 \%$ confidence range), based on individual spectral models for the most luminous sources, and, for all the others, adopting the median values of photon index and column density inferred from the modelled subsample $(\Gamma=1.8$ and total

$N_{\mathrm{H}}=3.0 \times 10^{21}$, respectively). We regard these luminosities as our best estimate, and use them for further analysis. Col. $(12)$ : Notes. A $=\mathrm{SN} 1979 \mathrm{C} ; \mathrm{B}=$ likely background galaxy at $z \approx 0.156 ; \mathrm{C}=$ likely foreground star; $\mathrm{D}=\mathrm{NED}$ Tully-Fisher distance possibly overestimated; $\mathrm{E}=$ candidate nuclear source of a small (satellite?) galaxy; $\mathrm{F}=$ likely quasar; $\mathrm{G}=$ known $\mathrm{BL}$ Lac at $z \approx 0.43$.

all their fluxes again with srcflux, and $\Gamma=1.8$, but this time with the assumption of $N_{\mathrm{H} \text {,tot }}=3.0 \times 10^{21} \mathrm{~cm}^{-2}$, for all the sources not already included in Table 3. In summary, the list of "corrected" 0.3-10 keV luminosities provided in Table 2, Col. (7), is our best estimate, combining the spectral fitting results for the most luminous sources and the revised srcflux estimates for all the others. On average, the corrected unabsorbed luminosities for sources observed in earlier Chandra cycles (before the soft-energy degradation) are a factor of 1.6-1.7 times higher than estimated when only Galactic line-of-sight absorption is assumed. In Cycle 18, the difference between $N_{\mathrm{H}}=3.0 \times 10^{21} \mathrm{~cm}^{-2}$ and $N_{\mathrm{H}}=3.0 \times 10^{20} \mathrm{~cm}^{-2}$ was reduced to a factor of $\approx 1.3$.

\subsubsection{Candidate Type IIn SN in IC 3322A}

CXOU J122541.70+071338.9, detected in IC 3322A (distance of $\approx 25 \mathrm{Mpc}$ ) on 2018 April 24 , is the most luminous ULX in the whole sample. Its X-ray spectrum (Table 3) suggests multi-temperature thermal plasma emission and an intrinsic column density of $\approx 2$ $\times 10^{22} \mathrm{erg} \mathrm{s}^{-1}$, for an intrinsic luminosity of $\approx 6 \times 10^{40} \mathrm{erg} \mathrm{s}^{-1}$. A 
spectrum dominated by X-ray thermal plasma at such a high luminosity is very unusual for a ULX. The X-ray spectrum is consistent with that of a young SN; more specifically, of a Type IIn, which explodes in a denser circumstellar medium. Based on the Supernova X-ray Database online catalog 9 (Ross \& Dwarkadas 2017), there are at least five core-collpase SNe (SN 1998Z, SN 1995N, SN 2005ip, SN 2005kd, SN 2006jd and SN 2010jl), detected at X-ray luminosities of a few $10^{40} \mathrm{erg} \mathrm{s}^{-1}$, even up to a few $10^{41} \mathrm{erg} \mathrm{s}^{-1}$, for several years after the explosion. See also Chandra et al. (2012, 2015) and Chandra (2018) for detailed discussions of the X-ray and multi-band properties of Type IIn SNe. The possibility that some of the brightest ULXs may be young $\mathrm{SNe}$ in a dense environment was discussed in other cases, for example for the brightest ULX in the Cartwheel galaxy (Pizzolato et al. 2010).

No core-collapse SNe have ever been reported in IC 3322A, but that is not strong evidence against our suggested interpretation. The peak absolute optical brightness of Type IIn SNe is broadly distributed between about $-17 \mathrm{mag}$ and $-21 \mathrm{mag}$, with a median value around -19 mag (Nyholm et al. 2020). The distance modulus of IC $3322 \mathrm{~A}$ is $\approx 32 \mathrm{mag}$. To that, we may add $10 \mathrm{mag}$ of visual extinction, based on the best-fitting value of $N_{\mathrm{H}} \approx 2 \times 10^{22} \mathrm{~cm}^{-2}$ (Predehl \& Schmitt 1995; Güver \& Özel 2009; Willingale et al. 2013). Thus, it is likely that the presumed SN was fainter than 20 mag in the optical, and would have been easily missed by large-area sky searches.

We used the Open SN Catalog ${ }^{10}$ catalogs (Guillochon et al. 2017 ) to check whether any of the point-like sources (even those fainter than $10^{39} \mathrm{erg} \mathrm{s}^{-1}$, not listed in Table 2) may correspond to a historical SN. There are 39 optically-identified SNe reported to occur in the 75 sample galaxies prior to at least one of the Chandra observations used for this study. Only three of them are detected in X-rays (Table 4). The most luminous of them is SN 1979C (a Type II L) in NGC 4321, for which we infer an X-ray luminosity of $\approx 6 \times 10^{38} \mathrm{erg} \mathrm{s}^{-1}$ when its spectrum (stacked over the six Chandra observations of this galaxy) is fitted with a two-temperature thermal plasma model. The best-fitting temperatures are $k T_{1}=0.27_{-0.08}^{+0.20}$ $\mathrm{keV}$ and $k T_{2}=1.3_{-0.2}^{+0.2} \mathrm{keV}$, and the best-fitting intrinsic column density is $\left(5.8_{-2.9}^{+2.7}\right) \times 10^{21} \mathrm{~cm}^{-2}$.

\subsubsection{Likely interlopers from other projected galaxies}

Two bright sources (Table 3) are associated with the nuclei of (apparently) small galaxies projected within the $D_{25}$ of a larger spiral (NGC 4492 and NGC 4567). We found no measurements for the redshifts of the two small companion galaxies. If the host galaxies are dwarf satellites at approximately the same distance as NGC 4492 and NGC 4567, respectively, the two X-ray sources would have a luminosity of several times $10^{39} \mathrm{erg} \mathrm{s}^{-1}$ (Table 3). However, they would not be classified as ULXs in the most common definition of this class, because they are nuclear sources. They would still be interesting as candidate intermediate-mass BHs in the nuclei of dwarf satellites. Instead, the bright source projected inside the $D_{25}$ of NGC 4698 (Tables 2, 3), known in the literature as XMMU $\mathrm{J} 124825.9+083020$ or WISE J124825.85+083020.4, is a BL Lac at redshift 0.43 , confirmed by optical spectroscopy (Foschini et al. 2002).
Another
curious
source
(Table
3) is
CXOU

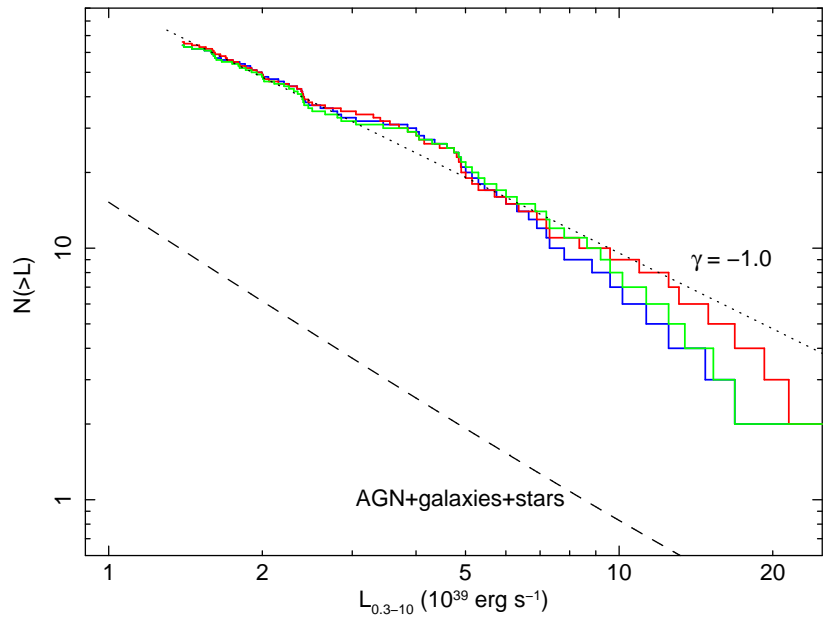

Figure 13. Cumulative LF in the $0.3-10 \mathrm{keV}$ band. See Col. (7), Table 2 for the data values. The datapoints include a subsample of ULXs modelled with free $N_{\mathrm{H}}$ and $\Gamma$ (Table 3), while for the fainter sources we fixed $\Gamma=1.8$ and $N_{\mathrm{H}, \text { tot }}=3.0 \times 10^{21} \mathrm{~cm}^{-2}$. The red histogram represents the luminosities inferred from the earliest exposure available for each galaxy with multiple observations; the green histogram shows the luminosities inferred from the latest available exposures for each galaxy; the blue histogram is for the longest available exposures. The small excess at the higher end of the earliest-look LF is the result of small-number statistics (namely, two ULXs with $L_{\mathrm{X}}>10^{40} \mathrm{erg} \mathrm{s}^{-1}$ in NGC 4527, detected only in the first observation of that galaxy). For graphical purposes only, the most luminous source (candidate SN) in the Virgo sample (Section 4.2.2) is falling outside the plotted region, at $L_{X} \approx 6 \times 10^{40} \mathrm{erg} \mathrm{s}^{-1}$. The black dashed line is the predicted contribution from the cosmic background plus foreground stars (Lehmer et al. 2012). The black dotted line shows that the lower end of the cumulative LF is consistent with a power-law of index -1 , with a downturn at the high-luminosity end.

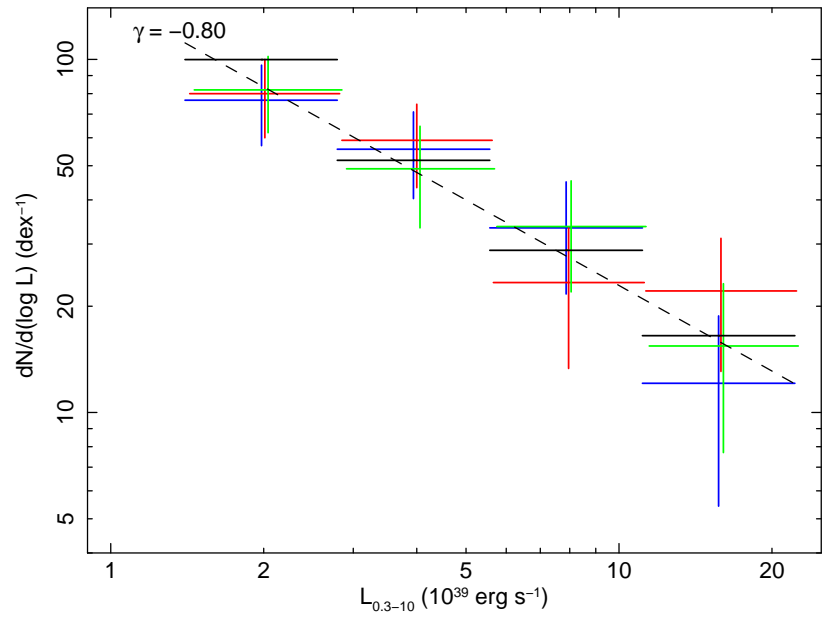

Figure 14. Differential LF in the $0.3-10 \mathrm{keV}$ band. For each of the four luminosity bins, of equal width $\Delta \log \left(L_{\mathrm{X}} / \mathrm{erg} \mathrm{s}^{-1}\right)=0.3$, the colours are defined as in Figure 13 (red, green and blue for the earliest, latest and longest observation, respectively). The relative position of the three coloured datapoints in each bin is offset by a very small amount along the $\mathrm{X}$ axis for visual clarity. The predicted background and foreground contributions have already been subtracted from each luminosity bin. The dashed black line is an indicative power-law model, with the form $d N / d(\log L) \propto L_{39}^{\gamma}$ with $\gamma=-0.80$; values of $\gamma \approx-0.8 \pm 0.2$ are consistent with the observed datapoints. For each luminosity bin, a black line marks the value predicted for that bin by the LF models of Lehmer et al. (2019) (LMXBs plus HMXBs).

9 https://kronos.uchicago.edu/snax/

$10 \mathrm{https}: / /$ sne.space/ 


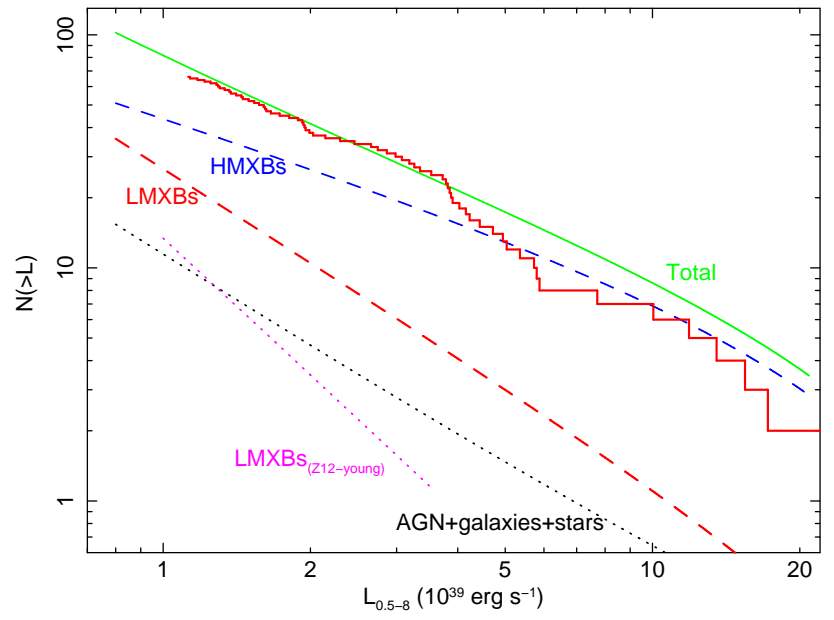

Figure 15. Comparison between the empirical cumulative LF from our survey, and the model components from Lehmer et al. (2019). Here, we have plotted all functions in the $0.5-8 \mathrm{keV}$ band, to facilitate the comparison. Conversions between $0.3-10 \mathrm{keV}$ and $0.5-8 \mathrm{keV}$ bands were done according to the best-fitting spectral models of the sources. Red histogram: cumulative ULX LF for the Virgo spiral sample (here we chose the distribution derived from the earliest observations). Dashed blue line: predicted contribution from HMXBs, for a star formation rate of $70 M_{\odot} \mathrm{yr}^{-1}$ (similar to the SFR estimated by B15). Dashed red line: predicted contribution from LMXBs in spiral disks, for a stellar mass of $1.5 \times 10^{12} M_{\odot}$. Dotted black line: predicted contribution from background galaxies, AGN, and foreground stars. Solid green line: sum of the previous three terms (HMXBs, LMXBs and bg/fg sources), which represents the expected observed luminosity function from our Virgo sample. Dotted magenta line: predicted contribution from LMXBs also for a stellar mass of $1.5 \times 10^{12} M_{\odot}$, but calibrated on the younger population of elliptical galaxies (Zhang et al. 2012). For graphical purposes only, the most luminous source (candidate $\mathrm{SN}$ ) in the Virgo sample (Section 4.2.2) is falling outside the plotted region, at $L_{X} \approx 6 \times 10^{40} \mathrm{erg} \mathrm{s}^{-1}$.

J122538.98+124000.8, projected inside the $D_{25}$ of NGC 4388, but also projected onto the spiral arm (not the nucleus) of an anonymous background galaxy at redshift $z \approx 0.156$, seen behind the Virgo galaxy. If this transient source belongs to NGC 4388, it is a run-of-the-mill ULX with $L_{\mathrm{X}} \approx 1.5 \times 10^{39} \mathrm{erg} \mathrm{s}^{-1}$; instead, if it belongs to the background spiral, it is a hyperluminous $\mathrm{X}$-ray source with $L_{\mathrm{X}} \approx 2.6 \times 10^{42} \mathrm{erg} \mathrm{s}^{-1}$ (in the 2001 June 8 observation), a strong intermediate-mass $\mathrm{BH}$ candidate. In a subsequent observation of the same galaxy a decade later (2011 December 7), the source was no longer detected (Table 2), which implies a decline of a factor of at least 40 .

\subsection{Properties of the ULX luminosity function}

LFs are ideally defined and quantified as instantaneous snapshots of an X-ray population. Collecting and including all sources (including transient ones) detected in several successive observations of the same galaxy leads to an over-estimate of the luminosity distribution. For this reason, when plotting the $0.3-10 \mathrm{keV}$ cumulative LF (Figure 13), we included only sources from one observation per galaxy. We compared the distribution obtained by selecting the earliest observation for each galaxy, the latest observation, and the longest observation. The three alternative histograms are consistent with each other at the low luminosity end. At the high end, the small excess of the earliest-look LF is the result of small-number statistics: two ULXs with $L_{\mathrm{X}}>10^{40} \mathrm{erg} \mathrm{s}^{-1}$ in NGC 4527, detected only in the first observation of that galaxy.
We used the $\log N-\log S$ curves in the Chandra Deep Field study of Lehmer et al. (2012) to estimate the number of contaminating sources (background galaxies, AGN, quasars, and foreground stars) in our survey. To do this, we calculated the expected number of such sources projected inside the $D_{25}$ of each of our sample galaxies, above a flux corresponding to a $0.3-10 \mathrm{keV}$ luminosity of $10^{39} \mathrm{erg} \mathrm{s}^{-1}$ for that galaxy (at its median NED distance, given in Table 1, Column 8). For most of the galaxies, the expected number is $<1$. Adding those numbers for all the galaxies, we estimate a total of $\approx 15$ contaminating sources above $10^{39} \mathrm{erg} \mathrm{s}^{-1}$ (Figure 13). In fact, the total number of 15 is likely to be an over-estimate, because it assumes that the count rates and fluxes of background AGN suffer no attenuation from photoelectric absorption passing through the halo or disk of the Virgo galaxies. With a similar method, we estimate the expected contamination at other luminosity levels; for example, only about 2 contaminating sources are expected above $\approx 5 \times 10^{39}$ $\mathrm{erg} \mathrm{s}^{-1}$. (A few candidate background/foreground sources have already been flagged in Table 2 and Table 3 ).

We divided the energy range between $1.4 \times 10^{39} \mathrm{erg} \mathrm{s}^{-1}$ and $2.2 \times 10^{40} \mathrm{erg} \mathrm{s}^{-1}(0.3-10 \mathrm{keV}$ band) into four equally spaced bins (in log scale), and computed the contribution of the three alternative LFs (earliest, latest and longest) in each bin. We then subtracted the expected background and foreground contributions in each bin from each of three observed distributions. This gives three alternative realizations of the differential LF of the point-like sources, corresponding to three different snapshots in time (Figure 14). As expected, there is a lot of scatter at the high-luminosity end, due to small-number statistics. A power-law with an index $\gamma=-0.80 \pm 0.20$ (defined as $d N / d(\log L) \propto L^{\gamma}$ ) is a good approximation of the slope of the differential LF. This is intermediate (as expected, given the mix of the two populations) between the slopes of $\approx-0.6$ for a pure HMXB population, and $\approx-1.3$ for LMXBs in that energy range (Lehmer et al. 2019). The normalization is also consistent (Figure 14) with the spiral galaxy models of Lehmer et al. (2019), for a total SFR $\approx 70 M_{\odot} \mathrm{yr}^{-1}$ (B15 value) and a total stellar mass $M_{* \text {,tot }}=1.5 \times 10^{12} M_{\odot}$. This does not imply that the SFR of $\approx 100$ $M_{\odot} \mathrm{yr}^{-1}$ inferred from the WISE photometry is wrong. It simply means that the SFR proxies (far-UV GALEX plus $24 \mu \mathrm{m}$ Spitzer luminosity) used by Lehmer et al. (2019) to calibrate the normalization of the HMXB population provide SFR values more similar to the $\mathrm{B} 15$ values than to the WISE-derived values.

Our empirical differential LF falls slightly below the model predictions at the low-luminosity end (Figure 14). This suggests that we are missing a few sources. This is expected. Sources with a $0.3-10 \mathrm{keV}$ luminosity of $\sim 1.5 \times 10^{39} \mathrm{erg} \mathrm{s}^{-1}$ and an absorbing column $N_{\mathrm{H}} \gtrsim$ a few times $10^{21} \mathrm{~cm}^{-2}$ have a substantial chance of missing our first selection cut (Table 2, Col. 6), depending on their photon index and on the Chandra observing cycle. For this work, we are not particularly concerned by this issue: ULXs in the luminosity range $\sim(1-1.5) \times 10^{39} \mathrm{erg} \mathrm{s}^{-1}$ are the upper end of the ordinary X-ray binary population; we are more interested in the high-luminosity end of the ULX population.

The total $0.3-10 \mathrm{keV}$ luminosity of the resolved $D_{25}$ population above $1.4 \times 10^{39} \mathrm{erg} \mathrm{s}^{-1}$ is $\sim(3.5-3.7) \times 10^{41} \mathrm{erg} \mathrm{s}^{-1}$ (depending on the choice of observation for galaxies with multiple exposures). About $10 \%$ of this luminosity comes from background AGN projected inside the $D_{25}$ regions. An extrapolation of the differential LF (Figure 14) with a power-law slope of $\gamma=-0.8 \pm 0.2$ down to $10^{36} \mathrm{erg} \mathrm{s}^{-1}$ predicts an additional contribution of $\approx 2-3 \times 10^{41} \mathrm{erg}$ $\mathrm{s}^{-1}$ from unresolved point sources. This value is not very sensitive to the choice of the lower limit, as most of the integrated emission comes from the high-luminosity sources. Thus, our best estimate for 
Table 3. Spectral parameters of the most luminous ULXs in Virgo $\left(L_{X} \gtrsim 4 \times 10^{39} \mathrm{erg} \mathrm{s}^{-1}\right)$

\begin{tabular}{|c|c|c|c|c|c|c|c|c|c|c|c|}
\hline Host Galaxy & R.A.(J2000) & Dec.(J2000) & ObsID & Obs. Date & $\begin{array}{c}N_{\mathrm{H}, \mathrm{Gal}} \\
\left(10^{22} \mathrm{~cm}^{-2}\right)\end{array}$ & $\begin{array}{c}N_{\mathrm{H}, \mathrm{int}} \\
\left(10^{22} \mathrm{~cm}^{-2}\right)\end{array}$ & $\Gamma$ & $N_{\mathrm{pl}}^{a}$ & C-stat/dof & $\begin{array}{c}F_{0.5-8}^{b} \\
\left(10^{-14} \mathrm{erg} \mathrm{cm}^{-2} \mathrm{~s}^{-1}\right)\end{array}$ & $\begin{array}{c}L_{0.3-10}^{c} \\
\left(10^{39} \mathrm{erg} \mathrm{s}^{-1}\right)\end{array}$ \\
\hline \multicolumn{12}{|c|}{ Most likely ULXs } \\
\hline NGC 4178 & 121244.51 & +1051 13.6 & 12748 & 2011-02-19 & {$[0.026]$} & $0.24_{-0.09}^{+0.09}$ & $1.47_{-0.21}^{+0.21}$ & $3.3_{-0.7}^{+0.9}$ & $206.9 / 235$ & $19.7_{-2.0}^{+2.2}$ & $6.0_{-0.5}^{+0.6}$ \\
\hline \multirow[t]{2}{*}{ NGC 4197} & 121440.29 & +054900.0 & 19420 & $2017-07-26$ & {$[0.015]$} & $0.23_{-0.23}^{+1.21}$ & $1.3_{-*}^{+2.5}$ & $0.6_{-0.4}^{+5.8}$ & $11.5 / 14$ & $4.3_{-2.6}^{+5.8}$ & $5.2_{-2.4}^{+5.9}$ \\
\hline & 121441.31 & +05 4903.9 & 19420 & $2017-07-26$ & {$[0.015]$} & $<0.8$ & $0.8_{-0.6}^{+0.8}$ & $0.30_{-0.16}^{+0.44}$ & $30.8 / 17$ & $4.6_{-2.0}^{+2.6}$ & $4.9_{-1.7}^{+3.8}$ \\
\hline NGC 4212 & 121539.59 & +135342.2 & 19395 & $2017-02-14$ & {$[0.026]$} & $0.19_{-0.19}^{+0.25}$ & $1.59_{-0.43}^{+0.46}$ & $2.7_{-1.0}^{+1.8}$ & $92.3 / 104$ & $14.0_{-2.5}^{+3.1}$ & $7.1_{-1.1}^{+1.8}$ \\
\hline NGC 4216 & 121550.12 & +1306 18.3 & 19391 & 2017-07-24 & {$[0.028]$} & $0.81_{-0.77}^{+1.13}$ & $2.01_{-0.96}^{+1.15}$ & $3.0_{-2.2}^{+10.2}$ & $26.8 / 36$ & $7.4_{-3.1}^{+3.0}$ & $4.8_{-2.0}^{+16.2}$ \\
\hline \multirow[t]{2}{*}{ NGC 4254} & 121856.10 & +14 2419.3 & 7863 & $2007-11-21$ & {$[0.026]$} & $0.10_{-0.09}^{+0.10}$ & $1.35_{-0.27}^{+0.28}$ & $8.8_{-2.2}^{+3.1}$ & $137.4 / 170$ & $64.3_{-8.8}^{+10.3}$ & $21.6_{-2.6}^{+3.2}$ \\
\hline & & & 17462 & 2015-02-16 & {$[0.026]$} & $0.27_{-0.14}^{+0.16}$ & $1.62_{-0.24}^{+0.25}$ & $3.6_{-0.9}^{+1.3}$ & $185.4 / 237$ & $17.3_{-1.7}^{+1.9}$ & $6.6_{-0.7}^{+0.8}$ \\
\hline NGC 4316 & 122239.54 & +09 2021.0 & 19400 & 2018-04-20 & {$[0.014]$} & $0.27_{-0.27}^{+0.53}$ & $2.44_{-0.91}^{+1.13}$ & $1.3_{-0.8}^{+2.7}$ & $22.9 / 34$ & $2.9_{-0.9}^{+1.3}$ & $5.4_{-2.4}^{+16.6}$ \\
\hline \multirow[t]{3}{*}{ NGC 4321} & 122254.14 & +154912.6 & 9121 & 2008-04-20 & {$[0.018]$} & $0.28_{-0.11}^{+0.12}$ & $3.46_{-0.55}^{+0.61}$ & $7.3_{-2.3}^{+3.6}$ & $101.5 / 112$ & $8.8_{-1.1}^{+1.2}$ & $14.4_{-6.3}^{+16.7}$ \\
\hline & & & 23141 & 2020-03-13 & {$[0.018]$} & $0.32_{-0.27}^{+0.33}$ & $3.11_{-0.72}^{+0.81}$ & $8.4_{-4.1}^{+9.1}$ & $54.7 / 75$ & $10.9_{-2.2}^{+3.0}$ & $14.0_{-7.5}^{+30.6}$ \\
\hline & 122254.78 & +154916.3 & 9121 & 2008-04-20 & {$[0.018]$} & $0.31_{-0.14}^{+0.16}$ & $2.28_{-0.44}^{+0.48}$ & $4.5_{-1.5}^{+2.4}$ & $87.2 / 115$ & $10.6_{-1.8}^{+2.3}$ & $6.9_{-1.5}^{+3.5}$ \\
\hline IC 3322A & 122541.70 & +071338.9 & 19401 & 2018-04-24 & {$[0.017]$} & $2.10_{-0.74}^{+1.27}$ & \multicolumn{2}{|c|}{ (NA-thermal source) $^{d}$} & $30.9 / 46$ & $3.9_{-0.9}^{+1.3}$ & $62.2_{-38.8}^{+69.8}$ \\
\hline NGC 4396 & 122601.81 & +153941.8 & 19417 & $2018-05-25$ & {$[0.020]$} & $0.73_{-0.48}^{+0.61}$ & $2.84_{-0.80}^{+0.92}$ & $9.6_{-5.7}^{+17.3}$ & $55.0 / 61$ & $10.2_{-2.2}^{+2.8}$ & $9.4_{-5.2}^{+31.0}$ \\
\hline NGC 4411B & 122649.48 & +085218.9 & 19429 & 2017-02-09 & {$[0.013]$} & $0.54_{-0.51}^{+0.72}$ & $2.39_{-1.03}^{+1.26}$ & $1.9_{-1.3}^{+5.2}$ & $31.6 / 28$ & $3.5_{-1.2}^{+1.7}$ & $5.5_{-2.7}^{+23.8}$ \\
\hline NGC 4496A & 123138.08 & +035642.2 & 16995 & $2015-07-26$ & {$[0.023]$} & $<0.20$ & $1.20_{-0.30}^{+0.52}$ & $4.8_{-1.1}^{+3.5}$ & $61.4 / 80$ & $44.2_{-10.3}^{+11.8}$ & $16.6_{-3.6}^{+5.2}$ \\
\hline \multirow[t]{2}{*}{ NGC 4501} & 123200.39 & +142442.4 & 2922 & 2002-12-09 & {$[0.027]$} & $0.31_{-0.12}^{+0.14}$ & $3.83_{-0.66}^{+0.77}$ & $4.7_{-1.6}^{+2.7}$ & $91.5 / 91$ & $4.7_{-0.7}^{+0.7}$ & $12.9_{-6.8}^{+22.5}$ \\
\hline & 123200.94 & +142502.6 & 2922 & $2002-12-09$ & {$[0.027]$} & $0.26_{-0.11}^{+0.12}$ & $2.20_{-0.39}^{+0.42}$ & $3.3_{-1.0}^{+1.5}$ & $130.2 / 127$ & $8.9_{-1.5}^{+1.8}$ & $6.0_{-1.1}^{+2.1}$ \\
\hline \multirow[t]{2}{*}{ NGC 4527} & 123410.94 & +02 3925.2 & 4017 & 2003-03-09 & {$[0.020]$} & $0.84_{-0.35}^{+0.46}$ & $1.23_{-0.46}^{+0.50}$ & $7.9_{-3.6}^{+7.6}$ & $99.1 / 126$ & $55.3_{-19.9}^{+22.1}$ & $21.4_{-3.3}^{+4.3}$ \\
\hline & 123411.43 & +02 3928.9 & 4017 & 2003-03-09 & {$[0.020]$} & $0.55_{-0.27}^{+0.35}$ & $1.22_{-0.45}^{+0.49}$ & $4.9_{-2.1}^{+4.2}$ & $71.0 / 99$ & $36.4_{-7.2}^{+8.8}$ & $13.3_{-2.2}^{+2.8}$ \\
\hline \multirow[t]{2}{*}{ NGC 4532} & 123418.89 & +06 2815.4 & 19407 & 2018-05-15 & {$[0.016]$} & $0.40_{-0.30}^{+0.37}$ & $1.73_{-0.49}^{+0.53}$ & $5.4_{-2.4}^{+4.8}$ & $89.3 / 99$ & $21.7_{-4.1}^{+4.9}$ & $7.3_{-1.4}^{+3.2}$ \\
\hline & 123420.34 & +06 2737.5 & 19407 & 2018-05-15 & {$[0.016]$} & $0.43_{-0.33}^{+0.43}$ & $2.97_{-0.79}^{+0.93}$ & $7.0_{-3.8}^{+10.1}$ & $37.7 / 55$ & $8.7_{-2.0}^{+3.5}$ & $7.3_{-4.0}^{+20.6}$ \\
\hline \multirow[t]{2}{*}{ NGC 4536} & 123426.16 & +021122.3 & 19387 & 2017-07-01 & {$[0.016]$} & $0.52_{-0.39}^{+0.49}$ & $1.57_{-0.57}^{+0.62}$ & $2.4_{-1.2}^{+2.8}$ & $86.9 / 89$ & $11.4_{-2.3}^{+3.0}$ & $5.1_{-1.0}^{+2.2}$ \\
\hline & 123436.93 & +020851.4 & 19387 & 2017-07-01 & {$[0.016]$} & $0.29_{-0.23}^{+0.28}$ & $3.00_{-0.58}^{+0.64}$ & $7.1_{-3.0}^{+5.9}$ & $64.9 / 92$ & $10.2_{-8.4}^{+2.3}$ & $9.8_{-4.6}^{+14.2}$ \\
\hline \multirow[t]{3}{*}{ NGC 4568} & 123633.04 & +111405.6 & 19389 & 2018-03-01 & {$[0.024]$} & $0.05_{-0.05}^{+0.37}$ & $0.82_{-0.34}^{+0.54}$ & $0.88_{-0.30}^{+0.87}$ & $68.6 / 75$ & $13.0_{-2.9}^{+3.2}$ & $6.7_{-1.5}^{+1.9}$ \\
\hline & & & 19396 & 2018-05-10 & {$[0.024]$} & $0.47_{-0.47}^{+0.72}$ & $1.36_{-0.63}^{+0.74}$ & $2.5_{-1.5}^{+4.2}$ & $52.0 / 66$ & $15.5_{-3.6}^{+4.6}$ & $9.0_{-2.0}^{+4.6}$ \\
\hline & 123633.22 & +111437.1 & 19389 & 2018-03-01 & {$[0.024]$} & $<0.16$ & $1.20_{-0.21}^{+0.21}$ & $1.09_{-0.29}^{+0.36}$ & $71.4 / 78$ & $10.2_{-2.0}^{+2.4}$ & $4.9_{-1.0}^{+1.3}$ \\
\hline \multirow[t]{2}{*}{ NGC 4579} & 123740.31 & +114727.8 & 807 & $2000-05-02$ & {$[0.031]$} & $0.11_{-0.03}^{+0.03}$ & $1.72_{-0.12}^{+0.12}$ & $7.4_{-0.8}^{+0.9}$ & $275.4 / 300$ & $35.7_{-2.4}^{+2.5}$ & $17.1_{-0.9}^{+1.0}$ \\
\hline & 123753.88 & +11 5020.4 & 807 & 2000-05-02 & {$[0.031]$} & $0.10_{-0.09}^{+0.11}$ & $2.01_{-0.43}^{+0.51}$ & $2.1_{-0.7}^{+1.1}$ & $92.5 / 92$ & $7.8_{-1.6}^{+2.0}$ & $4.0_{-0.7}^{+1.2}$ \\
\hline NGC 4607 & 124112.61 & +115350.2 & 19403 & 2018-05-09 & {$[0.025]$} & $0.82_{-0.62}^{+0.88}$ & $2.37_{-0.95}^{+1.12}$ & $4.6_{-3.1}^{+12.7}$ & $36.9 / 40$ & $7.6_{-2.1}^{+3.0}$ & $10.5_{-5.0}^{+40.1}$ \\
\hline \multicolumn{12}{|c|}{ Likely ULX but very uncertain distance } \\
\hline NGC 4480 & 123028.14 & +04 1440.9 & 19423 & 2016-12-06 & {$[0.021]$} & $0.17_{-0.17}^{+0.58}$ & $1.8_{-0.8}^{+1.2}$ & $0.96_{-0.55}^{+2.32}$ & $26.3 / 19$ & $4.1_{-1.5}^{+2.3}$ & $13.0_{-4.7}^{+22.4}$ \\
\hline \multicolumn{12}{|c|}{ Nuclei of apparently small galaxies: either dwarf satellites in Virgo, or unrelated background galaxies at unknown redshift } \\
\hline NGC 4492 & 123057.83 & +08 0435.2 & 15759 & $2014-04-25$ & [0.014] & $0.96_{-0.45}^{+0.54}$ & $1.87_{-0.53}^{+0.57}$ & $3.1_{-1.5}^{+3.4}$ & $89.6 / 108$ & $8.7_{-1.5}^{+1.8}$ & $\left(8.3_{-2.1}^{+5.8}\right)^{e}$ \\
\hline NGC 4567 & 123633.75 & +11 1610.6 & 19396 & 2018-05-10 & {$[0.024]$} & $<0.67$ & $1.22_{-0.51}^{+0.94}$ & $2.5_{-1.4}^{+4.2}$ & $29.6 / 28$ & $0.70_{-0.29}^{+1.57}$ & $\left(4.9_{-1.5}^{+2.5}\right)^{f}$ \\
\hline \multirow[b]{2}{*}{ NGC 4388} & & & Either a & LX in Virgo, & off-nuclear $\mathrm{h}$ & erluminous X-ray & source in a bacl & ground galaxy & $z \approx 0.155$ & & \\
\hline & 122538.98 & +124000.8 & 1619 & 2001-06-08 & {$[0.026]$} & $<0.08$ & $1.62_{-0.27}^{+0.28}$ & $0.57_{-0.09}^{+0.11}$ & $51.8 / 71$ & $3.4_{-0.8}^{+1.0}$ & $\begin{array}{l}\left(1.67_{-0.38}^{+0.51}\right)^{g} \\
\left(2652_{-565}^{+743}\right)^{h}\end{array}$ \\
\hline \multicolumn{12}{|c|}{ Background BL Lac at $z \approx 0.43$} \\
\hline NGC 4698 & 124825.87 & +083020.8 & 3008 & 2002-06-16 & {$[0.016]$} & $0.07_{-0.04}^{+0.04}$ & $2.05_{-0.19}^{+0.21}$ & $3.8_{-0.5}^{+0.7}$ & $164.5 / 210$ & $14.2_{-1.3}^{+1.5}$ & $\left(1.4_{-0.1}^{+0.2} \times 10^{5}\right)$ \\
\hline \multicolumn{12}{|c|}{ 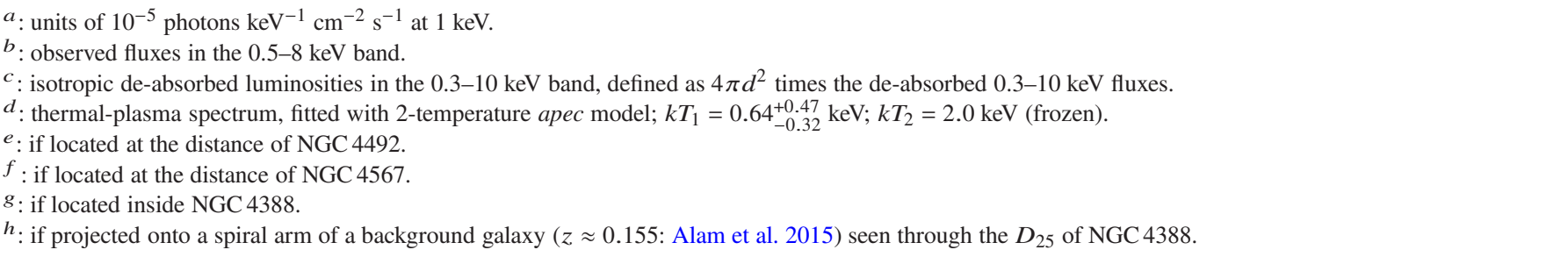 } \\
\hline
\end{tabular}

MNRAS 000, 1-24 (2021) 
Table 4. Historical SNe observed in the sample

\begin{tabular}{|c|c|c|c|c|c|c|c|}
\hline Host Galaxy & SN & R.A.(J2000) & Dec.(J2000) & Type & Peak Date & $\begin{array}{c}F_{0.5-8}^{a} \\
\left(10^{-16} \mathrm{erg} \mathrm{cm}^{-2} \mathrm{~s}^{-1}\right)\end{array}$ & $\begin{array}{c}L_{0.5-8}^{b} \\
\left(10^{37} \mathrm{erg} \mathrm{s}^{-1}\right)\end{array}$ \\
\hline NGC 4178 & $1963 \mathrm{I}$ & 121245.61 & +105131.0 & Ia & 1963-05-05 & $<7$ & $<1.5$ \\
\hline \multirow[t]{4}{*}{ NGC 4254} & $1967 \mathrm{H}$ & 121855.12 & +142440.3 & II & $1967-07-01$ & $<8$ & $<2.0$ \\
\hline & 1972Q & 121850.64 & +142636.3 & II P & $1972-12-17$ & $<8$ & $<2.0$ \\
\hline & 1986I & 121852.04 & +142444.1 & II P & $1986-05-17$ & $<8$ & $<2.0$ \\
\hline & $2014 \mathrm{~L}$ & 121848.68 & +142443.5 & Ic & $2014-01-26$ & $\left(20_{-15}^{+20}\right)^{c}$ & $\left(5_{-3}^{+5}\right)^{c}$ \\
\hline NGC 4302 & $1986 \mathrm{E}$ & 122141.21 & +143755.0 & II L & 1986-04-13 & $38_{-17}^{+26}$ & $16_{-7}^{+12}$ \\
\hline \multirow[t]{7}{*}{ NGC 4303} & $1926 \mathrm{~A}$ & 122154.15 & +042934.3 & II L & 1926-05-09 & $<10$ & $<2$ \\
\hline & $1961 \mathrm{I}$ & 122200.45 & +042813.5 & II & 1961-06-03 & $<10$ & $<2$ \\
\hline & $1964 \mathrm{~F}$ & 122153.02 & +042824.3 & II & $1964-06-13$ & $<10$ & $<2$ \\
\hline & $1999 \mathrm{gn}$ & 122157.02 & +042745.6 & II P & $1999-12-17$ & $<10$ & $<2$ \\
\hline & $2006 \mathrm{ov}$ & 122155.30 & +042916.7 & II P & $2006-11-24$ & $<15$ & $<3$ \\
\hline & 2008in & 122201.77 & +042847.5 & II P & $2008-12-26$ & $<15$ & $<3$ \\
\hline & $2014 \mathrm{dt}$ & 122157.57 & +042818.5 & Ia pec & 2014-10-29 & $<15$ & $<3$ \\
\hline NGC 4316 & 2003bk & 122242.14 & +092001.2 & II & $2003-02-28$ & $<37$ & $<33$ \\
\hline \multirow[t]{6}{*}{ NGC 4321} & 1901B & 122247.60 & +154925.0 & I & $1901-02-10$ & $<10$ & $<1.4$ \\
\hline & $1914 \mathrm{~A}$ & 122257.00 & +154730.0 & $?$ & 1914-03-02 & $<7$ & $<1$ \\
\hline & $1959 \mathrm{E}$ & 122258.91 & +154901.3 & I & $1959-02-21$ & $<7$ & $<1$ \\
\hline & $1979 \mathrm{C}$ & 122258.63 & +154751.7 & II L & $1979-04-15$ & $160_{-20}^{+20}$ & $63_{-11}^{+13}$ \\
\hline & $2006 \mathrm{X}$ & 122253.99 & +154833.1 & Ia & 2006-02-19 & $<7$ & $<1$ \\
\hline & $2020 \mathrm{oi}$ & 122254.96 & +154925.0 & Ic & $2020-01-07$ & $<100^{d}$ & $<15^{d}$ \\
\hline NGC 4402 & 1976B & 122611.91 & +130644.8 & I & 1976-04-05 & $<17$ & $<5$ \\
\hline NGC $4411 \mathrm{~b}$ & 1992ad & 122649.59 & +085238.2 & II & 1992-07-01 & $<30$ & $<7$ \\
\hline \multirow[t]{2}{*}{ NGC 4419} & $1984 \mathrm{~A}$ & 122655.71 & +150317.7 & Ia & $1984-01-17$ & $<30$ & $<10$ \\
\hline & $2012 \mathrm{cc}$ & 122656.81 & +150245.5 & II & 2012-04-29 & $50_{-30}^{+50}$ & $17_{-10}^{+17}$ \\
\hline \multirow[t]{2}{*}{ NGC 4424} & $1895 \mathrm{~A}^{e}$ & 122716.90 & +092505.0 & $?$ & 1895-03-16 & $<25$ & $<8$ \\
\hline & $2012 \mathrm{cg}$ & 122712.83 & +092513.2 & Ia & $2012-05-17$ & $<25$ & $<8$ \\
\hline NGC 4451 & $1985 \mathrm{G}$ & 122840.40 & +091540.0 & II P & $1985-03-17$ & $<20$ & $<17$ \\
\hline NGC 4496a & $1960 \mathrm{~F}$ & 123142.06 & +035647.9 & Ia & $1960-04-20$ & $<80$ & $<25$ \\
\hline NGC 4501 & $1999 \mathrm{cl}$ & 123156.01 & +142535.3 & Ia & 1999-06-15 & $<15$ & $<6$ \\
\hline \multirow[t]{3}{*}{ NGC 4527} & $1915 \mathrm{~A}$ & 123410.95 & +023903.5 & $?$ & $1915-03-20$ & $<20$ & $<5$ \\
\hline & $1991 \mathrm{~T}$ & 123410.17 & +023956.4 & Ia pec & $1991-04-28$ & $<20$ & $<5$ \\
\hline & 2004gn & 123412.10 & +023934.4 & Ic & 2004-12-01 & $<30$ & $<8$ \\
\hline NGC 4536 & 1981B & 123429.58 & +021159.3 & Ia & 1981-03-09 & $<30$ & $<8$ \\
\hline \multirow[t]{2}{*}{ NGC 4568} & 1990B & 123633.83 & +111429.8 & Ic & $1990-01-18$ & $<30$ & $<10$ \\
\hline & $2004 \mathrm{cc}$ & 123634.40 & +111432.8 & Ic & $2004-06-10$ & $<30$ & $<10$ \\
\hline \multirow[t]{2}{*}{ NGC 4579} & $1988 \mathrm{~A}$ & 123743.54 & +114819.4 & II P & 1988-01-19 & $<5$ & $<2$ \\
\hline & $1989 \mathrm{M}$ & 123740.72 & +114926.0 & Ia & $1989-06-28$ & $<5$ & $<2$ \\
\hline NGC 4639 & $1990 \mathrm{~N}$ & 124256.70 & +131523.7 & Ia & $1990-07-10$ & $<20$ & $<10$ \\
\hline NGC 4647 & $1979 \mathrm{~A}$ & 124329.14 & +113527.1 & I & 1979-01-15 & $<3$ & $<1$ \\
\hline
\end{tabular}

$a$ : observed fluxes in the $0.5-8 \mathrm{keV}$ band. To estimate the non-detection upper limits, we assumed a thermal-plasma apec model, with fixed temperature $k T=1 \mathrm{keV}$ and line-of-sight Galactic $N_{\mathrm{H}}$. For SN 2014L, SN 1986E, SN 2012cc, apec temperatures and column densities were left as free parameters in the fit, with Cash statistics. For SN 1979C, a two-temperature apec model was used (Section 4.2.2).

$b$ : de-absorbed luminosities in the $0.5-8 \mathrm{keV}$ band, based on the models mentioned above.

$c$ : likely due to extended thermal emission

$d$ : located in a region of strong diffuse emission

$e$ : first historical SN discovered outside the Local Group 
the background-subtracted point-source $0.3-10 \mathrm{keV}$ luminosity in the Virgo sample is $\sim 5-6 \times 10^{41} \mathrm{erg} \mathrm{s}^{-1}$, corresponding to $\sim 4.5-5$ $\times 10^{41} \mathrm{erg} \mathrm{s}^{-1}$ in the $0.5-8 \mathrm{keV}$ band.

First, we compare this extrapolated value with the integrated background-subtracted point-source galaxy luminosities estimated in Section 4.1. There, we had obtained a luminosity of $\approx 3.6 \times 10^{41}$ $\mathrm{erg} \mathrm{s}^{-1}$ in the $0.5-8 \mathrm{keV}$ band. However, for many of the galaxies with moderate or low emission (or short exposures) we had simply assumed a Galactic line-of-sight absorption in the conversion from count rates to fluxes and luminosities. Consequently, that value must be considered a lower limit. We have already seen (Section 4.2.1) that increasing the column density from $3 \times 10^{20} \mathrm{~cm}^{-2}$ to $3 \times 10^{21}$ $\mathrm{cm}^{-2}$ increases the inferred luminosity by 20 to 50 per cent (for a given count rate). Therefore, we conclude that the total luminosity estimates from the integration of the $D_{25}$ source regions and from the (extrapolated) point-source LF are consistent with each other. We had already noted (Section 4.1 ) that the value of $\approx 3.6 \times 10^{41}$ $\mathrm{erg} \mathrm{s}^{-1}$ appeared under-estimated for the given mass and SFR of the sample, and suggested that this under-estimation particularly affected galaxies with dusty disks, seen at high inclination.

Second, we compare the measured point-source luminosity with the model predictions (Lehmer et al. 2019), in the energy range where we have better constraints $\left(L_{\mathrm{X}} \gtrsim 1.2 \times 10^{39} \mathrm{erg} \mathrm{s}^{-1}\right.$ in the $0.5-8 \mathrm{keV}$ band). Direct sum of the ULX luminosities (minus the predicted AGN and foreground star fraction) gives a net $0.5-8 \mathrm{keV}$ luminosity of $\approx 2.7 \times 10^{41} \mathrm{erg} \mathrm{s}^{-1}$, but largely dependent on the small-number statistics of the most luminous sources. Over the same energy range, for an SFR of $70 M_{\odot} \mathrm{yr}^{-1}$ and a stellar mass of $1.5 \times 10^{12} M_{\odot}$, model predictions are $\approx 3.2 \times 10^{41} \mathrm{erg} \mathrm{s}^{-1}$, of which $\approx 2.5 \times 10^{41} \mathrm{erg} \mathrm{s}^{-1}$ from HMXBs and $\approx 0.7 \times 10^{41} \mathrm{erg} \mathrm{s}^{-1}$ from LMXBs. (The number of HMXBs per unit luminosity interval starts to dominate over the number of LMXBs at $L_{X} \approx 1.2 \times 10^{39}$ $\mathrm{erg} \mathrm{s}^{-1}$ ). As we noted earlier, the observed ULX luminosities are more consistent with the lower end of the published SFR values (B15 values) rather than the WISE-derived values.

We analyzed the $0.5-8 \mathrm{keV}$ cumulative luminosity distributions of observed sources and model predictions (Figure 15) to determine whether the small excess luminosity in the model of Lehmer et al. (2019) is significant. For sources below $\approx 4 \times 10^{39} \mathrm{erg} \mathrm{s}^{-1}$, the fiducial model follows our empirical distribution extremely well. Above that energy bin, the model is significantly above the observed LF. We suggest several alternative reasons for the discrepancy. The first possibility is that there is an imperfect match between the sector of the LF for which most of the sources have an individual spectral fit, and the sector where the luminosity was estimated with a fixed $N_{\mathrm{H}}$ and $\Gamma$. Splicing the two sub-samples together may create a normalization jump between them. The second possibility is that our simple (power-law) spectral models are still underestimating the luminosities of a few of the most luminous sources (more than for the fainter ones). For example, the most luminous ULXs might have a stronger thermal component below $1 \mathrm{keV}$, in addition to the power-law component, which was not included in the spectral models. More likely, our luminosity function may include a few ULXs with an intrinsic luminosity $L_{X} \gtrsim 4 \times 10^{39} \mathrm{erg} \mathrm{s}^{-1}$ but an absorption column $>10^{22} \mathrm{~cm}^{-2}$ (e.g., in dense star-forming regions or inside an edge-on galactic disk), which have been incorrectly placed in lower-luminosity bins. Finally, and perhaps more interestingly, the assumed shape of the model functions may not be correct. In Lehmer et al. (2019), both the LMXB and HMXB differential LFs in the ULX range were assumed to be unbroken power-laws (with different slopes) up to a cut-off at $5 \times 10^{40} \mathrm{erg} \mathrm{s}^{-1}$. While this may be justified for HMXBs, there is no sufficient evidence to say that it is also a good model for LMXBs. In fact, in the ULX sample studied by Lehmer et al. (2019), there are no LMXBs above a luminosity of $\approx 2 \times 10^{39} \mathrm{erg} \mathrm{s}^{-1}$. If the LMXB distribution has a cut-off at $L_{\mathrm{X}} \approx$ a few $\times 10^{39} \mathrm{erg} \mathrm{s}^{-1}$, it would help explain why our observed ULX distribution is consistent with the LMXB plus HMXB model function at the low luminosity end but follows the HMXB-only function at the high luminosity end (Figure 15).

\subsection{ULXs in early-type and late-type spiral galaxies}

Let us now compare the total number of ULXs found in our study with the numbers predicted by the models of Kovlakas et al. (2020) for spiral galaxies (valid from S0/a to Sm). The energy band used to define the ULX luminosity in their study is $0.5-8 \mathrm{keV}$. In the same band, we observed $\approx 60$ ULXs, after removing the expected background and foreground contributions. (We used the first observation of each galaxy for this estimate, but the number is approximately the same if we choose the longest observation or the latest observation.) Considering a mild effect of incompleteness around 1.0-1.3 $\times 10^{39}$ $\mathrm{erg} \mathrm{s}^{-1}$, hinted at in Section 4.3, the corrected number of ULXs at any given time is probably $\approx 65-70$, but we keep the estimate of 60 as a safe lower limit. The model of Kovlakas et al. (2020) predicts $N_{\mathrm{ULX}} \approx 0.45_{-0.09}^{+0.06} \times \mathrm{SFR}+3.3_{-3.2}^{+3.8} \times M_{*, 12}$, where $M_{*, 12}$ is in units of $10^{12} M_{\odot}$. For a meaningful comparison with our numbers, we need to consider the definition of SFR used by Kovlakas et al. (2020) for their calibration of the ULX scaling relation. In their case, the SFR was derived from the total infrared luminosity, primarily from the IRAS photometric measurements (calibration of Dale \& Henou 2002; Kennicutt \& Evans 2012) supplemented for some galaxies by the $12-\mu \mathrm{m}$ and $22-\mu \mathrm{m}$ WISE measurements. Therefore, for consistency, we use the WISE measurements of SFR for our galaxy sample rather than the B15 values (the latter being more biased in favour of UV- and $\mathrm{H} \alpha$-based SFR measurements). Namely, we take a total $\mathrm{SFR} \approx 86-104 M_{\odot} \mathrm{yr}^{-1}$, where the lower and higher values corresponds to the $22-\mu \mathrm{m}$ and $12-\mu \mathrm{m}$ estimates, respectively (Table 1 ). For this SFR range, the scaling relation of Kovlakas et al. (2020) predicts a best-fitting $N_{\mathrm{ULX}} \approx 44-52$. If we consider also the error range in the coefficients of the scaling relation, the predicted number of ULXs in our sample is $\approx 36-58$, lower than observed. Kovlakas et al. (2020) did point out that their ULX luminosities and hence ULX numbers are lower than those found in other surveys, including that of Swartz et al. (2011) (which is the most similar to the present work, for methodology). The difference was attributed to a different morphological composition of the samples, and a different conversion procedure between Chandra/ACIS count rates, fluxes and luminosities (Appendix C1 in Kovlakas et al. 2020).

More importantly, a comparison with the scaling of Kovlakas et al. (2020) is interesting because of their claimed strong difference between ULX rates in early-type spirals ( $\mathrm{S} 0 / \mathrm{a}$ to $\mathrm{Sbc}$ ) and late-type spirals (Sc or later). For the former, $N_{\mathrm{ULX}} \approx 0.16_{-0.08}^{+0.08} \times$ $\mathrm{SFR}+11.2_{-5.6}^{+5.2} \times M_{*, 12}$; for the latter, $N_{\mathrm{ULX}} \approx 0.98_{-0.20}^{+0.11} \times \mathrm{SFR}$. To test those relations, we split the Virgo spiral sample into two corresponding early-type and late-type subsamples, based on the NED classification (see also Table 1 and Figure 4). The earlytype-spiral sample includes 45 galaxies, with a total stellar mass of $\approx 1.2 \times 10^{12} M_{\odot}$ and a WISE-derived SFR of $\approx 55-66 M_{\odot} \mathrm{yr}^{-1}$; the late-type-spiral sample has 30 galaxies, with a stellar mass of $\approx 0.3 \times 10^{12} M_{\odot}$ and SFR $\approx 30-38 M_{\odot} \mathrm{yr}^{-1}$. For the parameters of the early-type-spiral sample, Kovlakas et al. (2020) predict a central value of $\approx 23-24$ ULXs $(\approx 16-31$ when we include the uncertainty on the scaling coefficients); our observed number is 39 . For 
the late-type-spiral sample, the predicted central value is $\approx 29-37$ ULXs ( $\approx 24-42$ with full uncertainty range); our observed number is 21 . We conclude that the scaling models of Kovlakas et al. (2020) underpredict the number of ULXs in early-type spirals and overpredict those in late-type ones, at least when applied to the Virgo sample.

\section{DISCUSSION AND SUMMARY}

We are conducting the first large-scale Chandra/ACIS survey of the spiral galaxy population in the Virgo cluster. This project complements and integrates the AMUSE-Virgo survey, which studied the early-type galaxy population of Virgo, a decade ago. We selected a sample of 75 galaxies, including all the largest and most active spirals ( $\approx 20$ late-type galaxies with $\mathrm{SFR} \gtrsim 1 M_{\odot}$ ), plus a selection of fainter ones. As detailed in Section 2.1, our sample is skewed towards brighter objects, in the sense that about 60 of our 75 galaxies have SFR $\gtrsim 0.3 \mathrm{M}_{\odot} \mathrm{yr}^{-1}$. This was done to provide a contrast with the early-type galaxy sample (Gallo et al. 2008; Plotkin et al. 2014; Graham \& Soria 2019) and to aid the discovery of luminous XRBs.

The total observing time was about $1.95 \mathrm{Ms}$ (combining new and archival observations), and for all galaxies in our sample we reached a point-source detection limit of $\approx 3 \times 10^{38} \mathrm{erg} \mathrm{s}^{-1}$ (or deeper, in a few cases).

In this first paper, we have outlined the general properties of the sample: the distance, morphological type, stellar mass and SFR. We have then presented the X-ray population properties of the sample galaxies, in relation to their stellar mass (for which we estimate a total of $\approx 1.5 \times 10^{12} M_{\odot}$ ) and SFR. For the SFR, we used the WISE $12 \mu \mathrm{m}$ and $22 \mu \mathrm{m}$ proxies and compared them with a collection of other SFR proxies (in particular, based on $\mathrm{H} \alpha$ and the far-UV) in the literature. Estimates vary between $\approx 70 M_{\odot} \mathrm{yr}^{-1}$ and $\approx 100$ $M_{\odot} \mathrm{yr}^{-1}$. Two-thirds of the galaxies can be described as active star-forming disks (dominated in X-rays by the HMXB population), while the others are early-type spirals, with low sSFR and an X-ray luminosity dominated by LMXBs.

We identified a sample of 85 ULXs that exceeded a $0.5-8$ $\mathrm{keV}$ luminosity of $10^{39} \mathrm{erg} \mathrm{s}^{-1}$ in at least one observation, and determined their coordinates. We fitted the spectral parameters for the most luminous sources ( 25 ULXs with $L_{X} \gtrsim 4 \times 10^{39} \mathrm{erg}$ $\mathrm{s}^{-1}$ ) and used their median power-law index and column density to convert count rates to luminosities for the rest of the ULXs, when they were too faint for individual fitting. We showed that the ULX luminosity distribution from our Virgo study is broadly consistent with the LF models from Lehmer et al. (2019), for a SFR $\sim 70 M_{\odot}$ $\mathrm{yr}^{-1}$ and a total stellar mass of $\approx 1.5 \times 10^{12} M_{\odot}$.

The model functions of Lehmer et al. (2019) predict a significant contribution (in number) from LMXBs: about half of the sources in the $\approx(1-1.5) \times 10^{39} \mathrm{erg} \mathrm{s}^{-1}$ range, and about one third of our total number of ULXs. At first sight, this appears to contradict the estimate from Plotkin et al. (2014) (based on their AMUSE-Virgo study), of only about 1 old-population ULX per $M_{*} \approx 1.6 \times 10^{11} M_{\odot}$, all of them with $L_{\mathrm{X}} \lesssim 2 \times 10^{39} \mathrm{erg} \mathrm{s}^{-1}$. (In our case, this would correspond to only about 10 old-population ULXs rather than about 30). In fact, there is no contradiction. The term "LMXB" is generally applied to a variety of X-ray binary populations that are proportional to stellar masses, but the normalization, break and slope of their LFs are substantially different, depending on age and environment (Zhang et al. 2012). In the disk of spiral galaxies, especially for sSFR $\lesssim 10^{-10} \mathrm{yr}^{-1}$, the number and luminosity of bright LMXBs (above a few times $10^{37} \mathrm{erg} \mathrm{s}^{-1}$ ) per unit mass is a few times higher than in old ellipticals. This is evident for example in the properties of the luminosity function in the disk of the nearby disk-dominated spiral M 83, where the LMXB component is at least as important as the HMXB component (Long et al. 2014). Most of the stellar mass in the AMUSE-Virgo survey is in massive elliptical galaxies (Table 1 in Gallo et al. 2010). Therefore, the scaling relations of (Zhang et al. 2012) are suitable to model the LMXB population mapped by that survey. Instead, the LMXB population in our sample includes a mix of spheroidal (spiral bulge) population for the early-type spirals, and pure disk population. Thus, we expect it to follow the scaling relation derived by Lehmer et al. (2019), at least up to a few times $10^{39} \mathrm{erg} \mathrm{s}^{-1}$.

The contribution of LMXBs to the higher-luminosity ULXs remains an open question. From the models of Lehmer et al. (2019) we expect four old-population sources above $L_{X} \approx 4 \times 10^{39} \mathrm{erg} \mathrm{s}^{-1}$ (0.5-8 keV band), compared with about 16 HMXBs. Instead, our Virgo ULX LF is consistent with the HMXB component alone. A cut-off of the LMXB population at $L_{\mathrm{X}} \approx 4 \times 10^{39} \mathrm{erg} \mathrm{s}^{-1}$ is one of the possible explanations we have proposed for the slight mismatch. Some theoretical population synthesis models (Wiktorowicz et al. 2017) do predict the formation of neutron star ULXs at least as luminous as $10^{40} \mathrm{erg} \mathrm{s}^{-1}$ during phases of steadily super-critical mass transfer from low-mass donors (main sequence, Hertzsprunggap stars, red giants, asymptotic giants, or white dwarfs), long after the end of star formation. The predicted number of old-population ULXs does, however, drop rapidly with age, decreasing by a factor of 20 from 1 to 5 Gyr after a star formation burst, and by another order of magnitude from 5 to 10 Gyr (Wiktorowicz et al. 2017). In addition to those steady mass-transfer systems, old-population ULXs should include also transient systems with a large (outer radius $\gtrsim 10^{12} \mathrm{~cm}$ ) accretion disk, subject to the thermal-viscous disk instability, which may exceed the Eddington limit during outburst (Hameury \& Lasota 2020).

In further work currently in preparation, we will illustrate and discuss the optical counterparts and stellar environments of the ULXs in this Virgo galaxy sample, based on the Next Generation Virgo Cluster Survey maps (Ferrarese et al. 2012) from the CanadaFrance-Hawaii Telescope. We will distinguish between younger and older populations, for the lower-luminosity and higher-luminosity subsets of ULXs, and for the early-type-spiral and late-type-spiral subsamples of galaxies. We will also discuss the metallicity effect on the high-luminosity end of the younger population.

Individual spectral studies of the most interesting Chandra sources will also be presented in follow-up work. Here, we have simply mentioned a few highlights. The most luminous ULX in the sample (a source in IC 3322A with $L_{\mathrm{X}} \approx 6 \times 10^{40} \mathrm{erg} \mathrm{s}^{-1}$ ) may not be an accreting compact object, as its X-ray spectrum is more consistent with that of a young $\mathrm{SN}$ (which could have been missed by optical/IR searches). Apart from that mysterious source, the three most luminous ULXs (in NGC 4254, NGC 4496A and NGC 4579) all reached a luminosity $L_{X} \approx 2 \times 10^{40} \mathrm{erg} \mathrm{s}^{-1}$, which is the well-known characteristic threshold above which ULXs become much rarer (Grimm et al. 2003; Swartz et al. 2004, 2011; Mineo et al. 2012a). At least three Chandra sources seen inside the $D_{25}$ of sample galaxies are likely to be the nuclear sources of smaller galaxies: further investigation is needed to determine whether those galaxies are also in Virgo (possibly satellites of their larger companions) or are a more distant background. 


\section{ACKNOWLEDGEMENTS}

RS acknowledges grant number 12073029 from the National Science Foundation of China. He is also grateful for support and hospitality from the Curtin Institute of Radio Astronomy (Perth, Australia) and from the Observatoire de Strasbourg during part of this work. MK acknowledges support from the French Centre National d'Études Spatiales (CNES). We are extremely grateful to the WXSC team (Team directors: Thomas Jarrett and Russ Taylor) for their support and their sharing of results in advance of their catalog publication. We thank Fabien Grisé, Manfred W. Pakull, PierreAlain Duc, Kinwah Wu, for their comments and suggestions, which improved the quality and the chances of success of our Chandra Large Program proposal, and Benjamin L. Davis for his expert advice on galaxy structures and scaling relations. We also thank Luca Cortese, Pat Coté, Jean-Charles Cuillandre and Stephen Gwyn, who helped us access the NGVS dataset. Finally, we thank the anonymous referee for their careful reading of the first version of this paper, and their insightful comments and suggestions.

\section{DATA AVAILABILITY}

The Chandra datasets used for this work are all available for download from the public archives. Reprocessed data can be provided upon request.

\section{REFERENCES}

Alam S., et al., 2015, ApJS, 219, 12

Ambrosi E., Zampieri L., 2018, MNRAS, 480, 4918

Arnaud K.A., 1996, ASPC, 101, 17

Artale M.C., Tissera, P.B., Pellizza L.J., 2015, MNRAS, 448, 3071

Artale M.C., Giacobbo N., Mapelli M., Esposito P., 2019, IAUS, 346, 332

Bachetti M., et al., 2014, Natur, 514, 202

Bartalucci I., Mazzotta P., Bourdin H., Vikhlinin A., 2014, A\&A, 566, 25

Belczynski K., Holz D.E., Bulik T., O’Shaughnessy R., 2016, Natur, 534, 512

Bell E.F., McIntosh D.H., Katz N., Weinberg M.D., 2003, ApJS, 149, 289

Binggeli B., Sandage A., Tarenghi M., 1984, AJ, 89, 64

Binggeli B., Tammann G.A., Sandage A., 1987, AJ, 94, 251

Blackburn J.K., 1995, in ASP Conf. Ser., Vol. 77, Astronomical Data Analysis Software and Systems IV, ed. R.A. Shaw, H.E. Payne, and J.J.E. Hayes (San Francisco: ASP), 367.

Boroson B., Kim D.-W., Fabbiano G., 2011, ApJ, 729, 12

Boselli A., Gavazzi G., 2006, PASP, 118, 517

Boselli A., Lequeux J., Gavazzi, G., 2002, A\&A, 384, 33

Boselli A., Fossati M., Gavazzi G., Ciesla L., Buat V., Boissier S., Hughes T.M., 2015, A\&A, 579, 102

Brorby M., Kaaret P., Prestwich A., 2014, MNRAS, 441, 2346

Cash W., 1979, ApJ, 228, 939

Chandra P., 2018, SSRv, 214, 27

Chandra P., Chevalier R.A., Chugai N., Fransson C., Soderberg A.M., 2015, ApJ, 810, 32

Chandra P., Chevalier R.A., Chugai N., Fransson C., Irwin C.M., Soderberg A.M., Chakraborti S., Immler S., 2012, ApJ, 755, 110

Chilingarian I.V., Katkov I.Y., Zolotukhin I.Y., Grishin K.A., Beletsky Y., Boutsia K., Osip D.J., 2018, ApJ, 863, 1

Cluver M.E., et al., 2014, ApJ, 782, 90

Cluver M.E., Jarrett T.H., Dale D.A., Smith J.-D.T., August T., Brown M.J.I., 2017, ApJ, 850, 68

Côté P., et al., 2004, ApJS, 153, 223

Dale D.A., Helou G., 2002, ApJ, 576, 159

de Grijs R., Bono G., 2020, ApJS, 246, 3

de Vaucouleurs G., 1959, HDP, 53, 275 de Vaucouleurs G., de Vaucouleurs A., Corwin H.G.,Jr., Buta R.J., Paturel G., Fouque P., 1991, Third Reference Catalogue of Bright Galaxies

El Mellah I., Sundqvist J.O., Keppens R., 2019, A\&A, 622, L3

Ferrarese L., et al., 2012, ApJS, 200, 4

Foschini L., et al., 2002, A\&A, 396, 787

Fragos T., et al., 2013, ApJ, 764, 41

Fragos T., Lehmer B.D., Naoz S., Zezas A., Basu-Zych A., 2013b, ApJ, 776, L31

Fragos T., Linden T., Kalogera V., Sklias P., 2015, ApJ, 802, L5

Freeman P., Doe S., Siemiginowska A., 2001, Proc. SPIE, 4477, 76

Fruscione A., et al., 2006, SPIE, 6270, 1

Gallo E., Treu T., Jacob J., Woo J.-H., Marshall P.J., Antonucci R., 2008, ApJ, 680, 154

Gallo E., Treu T., Marshall P.J., Woo J.-H., Leipski C., Antonucci R., 2010, ApJ, 714, 25

Gavazzi G., Boselli A., Scodeggio M., Pierini D., Belsole E., 1999, MNRAS, 304,595

Gavazzi G., Boselli A., Pedotti P., Gallazzi A., Carrasco L., 2002, A\&A, 396, 449

Gavazzi G., Boselli A., Donati A., Franzetti P., Scodeggio M., 2003, A\&A, 400,451

Gilfanov M., 2004, MNRAS, 349, 146

Gladstone J.C., Copperwheat C., Heinke C.O., Roberts T.P., Cartwright T.F., Levan A.J., Goad M.R., 2013, ApJS, 206, 14

Graham A.W., 2019, MNRAS, 487, 4995

Graham A.W., Soria R., 2019, MNRAS, 484, 794

Graham A.W., Soria R., Davis B.L., 2019, MNRAS, 484, 814

Graham A.W., Soria R., Davis B.L., Kolehmainen M., Maccarone T., MillerJones J., Motch C., Swartz D.A., 2021, ApJ, 923, 246

Grimm H.-J., Gilfanov M., Sunyaev R., 2003, MNRAS, 339, 793

Guillochon J., Parrent J., Kelley L.Z., Margutti R., 2017, ApJ, 835, 64

Güver T., Özel F., 2009, MNRAS, 400, 2050

HI4PI Collaboration, et al., 2016, A\& A, 594, 116

Hameury J.-M., Lasota J.-P., 2020, A\&A, 643, 171

Hong J.S., et al., 2016, ApJ, 825, 132

Huang T., Goto T., Hashimoto T., Oi N., Matsuhara H., 2017, MNRAS, 471, 4239

Jarrett T.H., Chester T., Cutri R., Schneider S.E., Huchra J.P., 2003, AJ, 125, 525

Jarrett T.H., Cluver M.E., Brown M.J.I., Dale D.A., Tsai C.W., Masci F., 2019, ApJS, 245, 25

Jarrett T.H., et al., 2013, AJ, 145, 6

Jarrett T.H., et al., 2017, ApJ, 836, 182

Joye W.A., Mandel E., 2003, in Astronomical Data Analysis Software and Systems XII ASP Conference Series, H.E. Payne, R.I. Jedrzejewski, and R.N. Hook, eds., Vol. 295, p. 489

Kaaret P., 2014, MNRAS, 440, L26

Kaaret P., Feng H., Roberts T.P., 2017, ARA\&A, 55, 303

Kalberla P.M.W., Burton W.B., Hartmann D., Arnal E.M., Bajaja E., Morras R., Pöppel W.G.L., 2005, A\&A, 440, 775

Kashibadze O.G., Karachentsev I.D., Karachentseva V.E., 2018, Astrophys.Bull., 73, 124

Kashibadze O.G., Karachentsev I.D., Karachentseva V.E., 2020, A\&A, 635, 135

Kennicutt R.C., 1998, ARA\&A, 36, 189

Kennicutt R.C., Evans N.J., 2012, ARA\&A, 50, 531

Khan F.M., Mirza M.A., Holley-Bockelmann K., 2020, MNRAS, 492, 256

Kim D.-W., Fabbiano G., 2004, ApJ, 611, 846

Kim D.-W., Fabbiano G., 2010, ApJ, 721, 1523

Kim S., et al., 2014, ApJS, 215, 22

Kovlakas K., Zezas A., Andrews J.J., Basu-Zych A., Fragos T., Hornschemeier A., Lehmer B., Ptak A., 2020, MNRAS, 498, 4790

Kroupa P., 2001, MNRAS, 322, 231

Kruckow M.U., Tauris T.M., Langer N., Kramer M., Izzard R.G., 2018, MNRAS, 481, 1908

Lasota J.-P., King A.R, Dubus G., 2015, ApJ, 801, L4

Lau R.M., 2019, ApJ, 878, 71 
Lehmer B.D., Alexander D.M., Bauer F.E., Brandt W.N., Goulding A.D., Jenkins L.P., Ptak A., Roberts T.P., 2010, ApJ, 724, 559

Lehmer B.D., et al., 2012, ApJ, 752, 46

Lehmer B.D., et al., 2016, ApJ, 825, 7

Lehmer B.D., et al., 2019, ApJS, 243, 3

Lehmer B.D., et al., 2021, ApJ, 907, 17

Li Z., Wang Q.D., 2007, ApJ, 668, L39

Li Z., et al., 2011, ApJ, 730, 84

Long K.S., Kuntz K.D., Blair W.P., Godfrey L., Plucinsky P.P., Soria R., Stockdale C., Winkler P.F., 2014, ApJS, 212, 21

López K.M., Heida M., Jonker P.G., Torres M.A.P., Roberts T.P., Walton D.J., Moon D.-S., Harrison F.A., 2020, MNRAS, 497, 917

Maccarone T.J., Kundu A., Zepf S.E., Rhode K.L., 2007, Natur, 445, 183

Maccarone T.J., Kundu A., Zepf S.E., Rhode K.L., 2010, MNRAS, 409, L84

Meidinger N., et al., 2020, Proceedings of the SPIE, Volume 11444, id. $114440 \mathrm{~T}$

Messier C., 1781, Catalogue des Nébuleuses et des Amas d'Étoiles, Connoissance des Temps ou des Mouvements Célestes, p. 227

Mezcua M., Civano F., Marchesi S., Suh H., Fabbiano G., Volonteri M., 2018, MNRAS, 478, 2576

Miller B., Gallo E., Treu T., Woo J.-H., 2012, ApJ, 745, L13

Mineo S., Gilfanov M., Sunyaev R., 2012a, MNRAS, 419, 2095

Mineo S., Gilfanov M., Sunyaev R., 2012b, MNRAS, 426, 1870

Mingo B., et al., 2016, MNRAS, 462, 2631

Mirabel I.F., Dijkstra M., Laurent P., Loeb A., Pritchard J.R., 2011, A\&A, 528,149

Misra D., Fragos T., Tauris T.M., Zapartas E., Aguilera-Dena D.R., 2020, A\&A, 642, 174

Mushtukov A.A., Ingram A., Middleton M., Nagirner D.I., van der Klis M., 2019, MNRAS, 484, 687

Nyholm A., et al., 2020, A\&A, 637, 73

Pavlovskii K., Ivanova N., Belczynski K., Van K.X., 2017, MNRAS, 465, 2092

Pintore F., Zampieri L., Wolter A., Belloni T., 2014, MNRAS, 439, 3461

Pintore F., Zampieri L., Stella L., Wolter A., Mereghetti S., Israel G.L., 2017, ApJ, 836, 113

Pizzolato F., Wolter A., Trinchieri G., 2010, MNRAS, 406, 1116

Planck Collaboration XL, 2016, A\&A, 596, 101

Plotkin R.M., Gallo E., Miller B.P., Baldassare V.F., Treu T., Woo J.-H., 2014, ApJ, 780, 6

Predehl P., Schmitt J.H.M.M., 1995, A\&A, 500, 459

Prestwich A.H., Tsantaki M., Zezas A., Jackson F., Roberts T.P., Foltz R., Linden T., Kalogera V., 2013, ApJ, 769, 92

Qiu Y., et al., 2019, ApJ, 877, 57

Quast M., Langer N., Tauris T.M., 2019, A\&A, 628, 19

Ranalli P., Comastri A., Setti G., 2003, A\&A, 399, 39

Revnivtsev M., Churazov E., Sazonov S., Forman W., Jones C., 2007, A\&A, 473,783

Ricarte A., Tremmel M., Natarajan P., Zimmer C., Quinn T., 2021, MNRAS, 503,6098

Ross M., Dwarkadas V.V., 2017, AJ, 153, 246

Salpeter E.E., 1955, ApJ, 121, 161

Shaya E.J., Tully R.B., Hoffman Y., Pomarede D., 2017, ApJ, 850, 207

Spera M., Mapelli M., Giacobbo N., Trani A.A., Bressan A., Costa G., 2019, MNRAS, 485, 889

Steele M.M., Zepf S.E., Maccarone T.J., Kundu A., Rhode K.L., Salzer J.J., 2014, ApJ, 785, 147

Sutton A.D., Roberts T.P., Middleton M.J., 2013, MNRAS, 435, 1758

Swartz D.A., Ghosh K.K., Tennant A.F., Wu K., 2004, ApJS, 154, 519

Swartz D.A., Soria R., Tennant A.F., Yukita M., 2011, ApJ, 741, 49

Tao L., Feng H., Grisé F., Kaaret P., 2011, ApJ, 737, 81

Walton D.J., Roberts T.P., Mateos S., Heard, V., 2011, MNRAS, 416, 1844

Walton D.J., et al., 2018, ApJ, 856, 128

Wiktorowicz G., Sobolewska M., Lasota J.-P., Belczynski K., 2017, ApJ, 846, 17

Wiktorowicz G., Lasota J.-P., Middleton M., Belczynski K., 2019, ApJ, 875, 53
Wiktorowicz G., Lasota J.-P., Belczynski K., Lu Y., Liu J.-F., Iłkiewicz K., 2021, ApJ, 918, 60

Willingale R., Starling R.L.C., Beardmore A.P., Tanvir N.R., O’Brien P.T., 2013, MNRAS, 431,394

Wright E.L., et al., 2010, AJ, 140, 1868

Zhang Z., Gilfanov M., Bogdán Á., 2012, A\&A, 546, 36

This paper has been typeset from a $\mathrm{T}_{\mathrm{E}} \mathrm{X} / \mathrm{L} \mathrm{T} \mathrm{E} \mathrm{X}$ file prepared by the author. 